\title{
Demonstration of Detection and Ranging Using Solvable Chaos
}

\author{
Ned J. Corron*a, Mark T. Stahl ${ }^{\mathrm{b}}$, and Jonathan N. Blakely ${ }^{\mathrm{a}}$ \\ ${ }^{a}$ Charles M. Bowden Laboratory, U. S. Army AMRDEC, Redstone Arsenal, Alabama 35898 USA \\ ${ }^{\mathrm{b}}$ NASA Marshall Space Flight Center, Alabama 35812 USA
}

\begin{abstract}
Acoustic experiments demonstrate a novel approach to ranging and detection that exploits the properties of a solvable chaotic oscillator. This nonlinear oscillator includes an ordinary differential equation and a discrete switching condition. The chaotic waveform generated by this hybrid system is used as the transmitted waveform. The oscillator admits an exact analytic solution that can be written as the linear convolution of binary symbols and a single basis function. This linear representation enables coherent reception using a simple analog matched filter and without need for digital sampling or signal processing. An audio frequency implementation of the transmitter and receiver is described. Successful acoustic ranging measurements are presented to demonstrate the viability of the approach.
\end{abstract}

Keywords: chaos, matched filter, radar, acoustic ranging, solvable chaos

\section{INTRODUCTION}

The wide bandwidth and aperiodic properties of chaos naturally suggest benefits for high-resolution, unambiguous ranging in radar, sonar, and ladar systems [1-13]. An obvious, conventional approach might be to substitute chaos for the noise source in random-signal radar. In such a system, a segment of the transmitted waveform is sampled and stored, using a resolution defined by the signal bandwidth and the Nyquist sampling criterion. The stored signal is then used in a correlation receiver to detect a return signal and determine time of flight. The cross-correlations are usually done digitally, using a digital signal processor (DSP) and fast-Fourier transforms (FFT). In this approach, the distinguishing properties of a chaotic waveform are not used: chaos is simply a wide-bandwidth, random source.

In contrast, we recently developed an alternative approach to detection and ranging that truly exploits the properties of a chaotic waveform to alleviate the most expensive parts of random-signal radar-i.e., sampling, digital memory, and digital signal processor - while still maintaining the performance of a correlation receiver [13]. This new approach uses chaotic waveforms generated by an analytically solvable nonlinear oscillator comprising an ordinary differential equation and a discrete switching state [14-15]. This hybrid oscillator admits an exact solution, which can be written as the linear convolution of a symbolic dynamics and a basis function. This analytic representation is significant since it enables coherent reception using a simple analog matched filter and only a few stored symbols.

In this paper, we present recent acoustic experiments that demonstrate this approach to ranging and detection. For these experiments, an amplified speaker emits an audio-frequency waveform generated by an electronic realization of the hybrid oscillator. The transmitted waveform sounds like noise. A complementary receiver circuit incorporates a matched filter for the chaotic waveform, which is mathematically equivalent to a correlation receiver. At repeated intervals, a sequence of symbols detected in the symbolic dynamics of the emitted waveform is captured, thereby defining a transmitted signal for ranging. The captured symbol sequence is provided to the receiver, where it defines the matched filter for the transmitted signal. Practically, the symbols define weights applied to elements of a microphone array, the outputs of which are summed and passively filtered. The output of the matched filter is a continuous signal that is proportional to the cross-correlation of the transmitted and received signal. In operation, a consistent peak in the output of the matched filter is observed, which indicates a detected target. Ranging is derived by the time of flight for the consistent peak.

The entire experimental system is realized using simple analog and digital electronic circuit components. Importantly, the receiver does not require waveform sampling or digital signal processing for detection. Real-time measurements using only an oscilloscope provide visible evidence of detection and ranging with the system.

*ned.corron@us.army.mil; phone 1 (256) 876-1860 


\section{HYBRID CHAOTIC OSCILLATOR}

The central element of the ranging demonstration system is the audio frequency oscillator shown schematically in Fig. 1. This electronic oscillator is a physical realization of a chaotic system previously considered by Tsubone and Saito [14] and Corron et al. [15]. This oscillator is a hybrid system, containing both an analog harmonic oscillator and digital logic circuits. For the acoustic system, we implemented the oscillator in an electronic circuit operating at roughly $10 \mathrm{kHz}$. The circuit is constructed using discrete analog and digital components on a solderless breadboard.

The dynamics of the oscillator are equivalent to a dimensionless hybrid model including a continuous scalar state $v(t)$ and a discrete state $s(t)$. The continuous-time dynamics are described by the differential equation

$$
\frac{d^{2} v}{d t^{2}}-2 \beta u \frac{d v}{d t}+\left(\omega^{2}+\beta^{2}\right) \cdot\left(v-v_{s}\right)=0
$$

where $\omega=2 \pi$ and $0<\beta \leq \ln 2$. Transitions in the discrete state are defined by the guard condition

$$
\frac{d v}{d t}(t)=0 \Rightarrow v_{s}(t)= \begin{cases}+1 & v \geq 0 \\ -1 & v<0\end{cases}
$$

meaning $v_{s}(t)$ is set to the sign of $v(t)$ whenever its time derivative vanishes, and $v_{s}(t)$ maintains this value until the next critical point. This oscillator admits an exact, analytic solution

$$
\begin{aligned}
& v(t)=\sum_{m=-\infty}^{\infty} s_{m} \cdot P(t-m) \\
& v_{s}(t)=\sum_{m=-\infty}^{\infty} s_{m} \cdot R(t-m)
\end{aligned}
$$

where each $s_{m}= \pm 1$ and $P(t)$ and $R(t)$ are fixed basis functions. In the solution, each symbol $s_{m}$ modulates the fixed basis functions $P(t)$ and $R(t)$ centered at time $t=m$. Thus, it is correct to think of the symbol $s_{m}$ as the information emitted by the oscillator at time $t=m$, and that the oscillator emits one symbol with each unit of time. The fixed basis functions are

$$
P(t)=\left\{\begin{array}{cc}
\left(1-e^{-\beta}\right) e^{\beta t}\left(\cos \omega t-\frac{\beta}{\omega} \sin \omega t\right), & t<0 \\
1-e^{\beta(t-1)}\left(\cos \omega t-\frac{\beta}{\omega} \sin \omega t\right), & 0 \leq t<1 \\
0, & 1 \leq t
\end{array}\right.
$$

and

$$
R(t)=\left\{\begin{array}{lc}
0, & t<0 \\
1, & 0 \leq t<1 \\
0, & 1 \leq t
\end{array}\right.
$$

which are shown in Fig. 2 for $\beta=\ln 2$. 


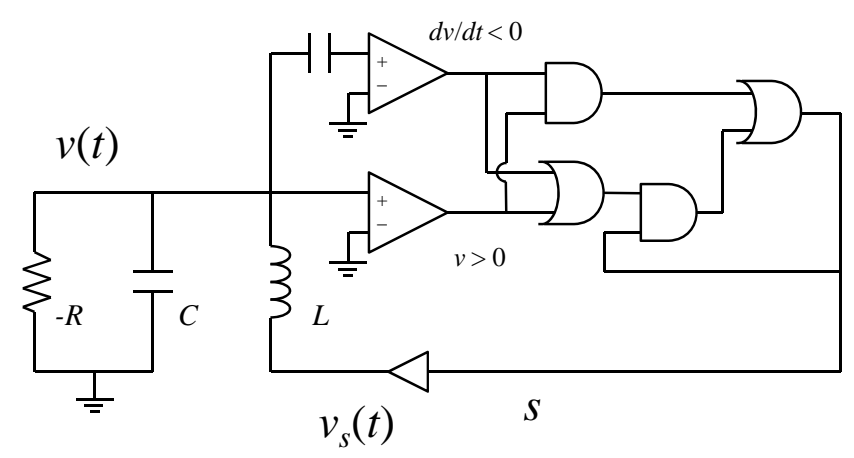

Figure 1. Exactly solvable chaotic oscillator.

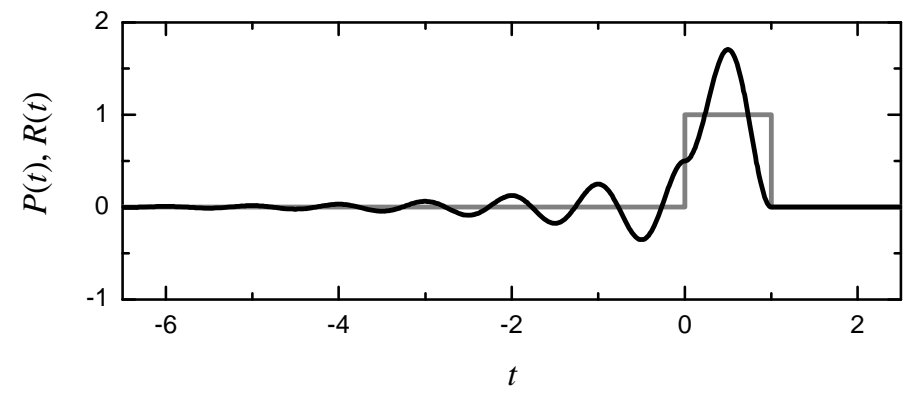

Figure 2. Analytic basis functions $P(t)$ (black) and $\mathrm{R}(\mathrm{t})$ (gray) for the oscillator model with $\beta=\ln 2$.

\section{TRANSMITTER}

The existence of fixed basis functions can be exploited for efficiently sampling the transmitted signal and storing a reference waveform. Here we show a transmitter design that achieves this important functionality.

The complete transmitter is shown in Fig. 3. At the center of the transmitter is the chaotic oscillator. The transmitted signal is the continuous state of the free-running chaotic oscillator, which is amplified and emitted by a conventional speaker. The additional circuitry at the bottom of the transmitter schematic derives a clock signal from the regular return times of the oscillator. The clock signal drives a binary shift register, which uses the signal $s(t)$ for the data input. A divide-by- $N$ counter circuit provides a signal to alternately enable and disable the shift register. For our experimental system, we typically use the value $N=1024$.

In operation, the free-running oscillator generates a chaotic waveform that is continuously emitted from the speaker. While the shift register is enabled, the symbolic logic state $s(t)$ is sampled for each return and stored in the shift register. At any time, a fixed number of the most recent values of the logic state $s(t)$ are stored, which correspond to a sequence of amplitudes $s_{m}$ generated by the free-running oscillator. Although the figure only shows an eight-bit shift register, for the acoustic system we used a twelve-bit register.

After shifting $N$ successive returns through the register, the shift register is disabled by the signal from the divide-by- $N$ circuit. When disabled, the contents of the shift register are locked, thereby storing symbols that identify the signal transmitted just prior to the disabling transition. These stored symbols effectively define a reference waveform to use for detection and ranging in a correlation receiver. Compared to the usual Nyquist sampling criteria, this symbolic representation provides at an order of magnitude reduction in the sampling and storage requirements for the reference waveform. 


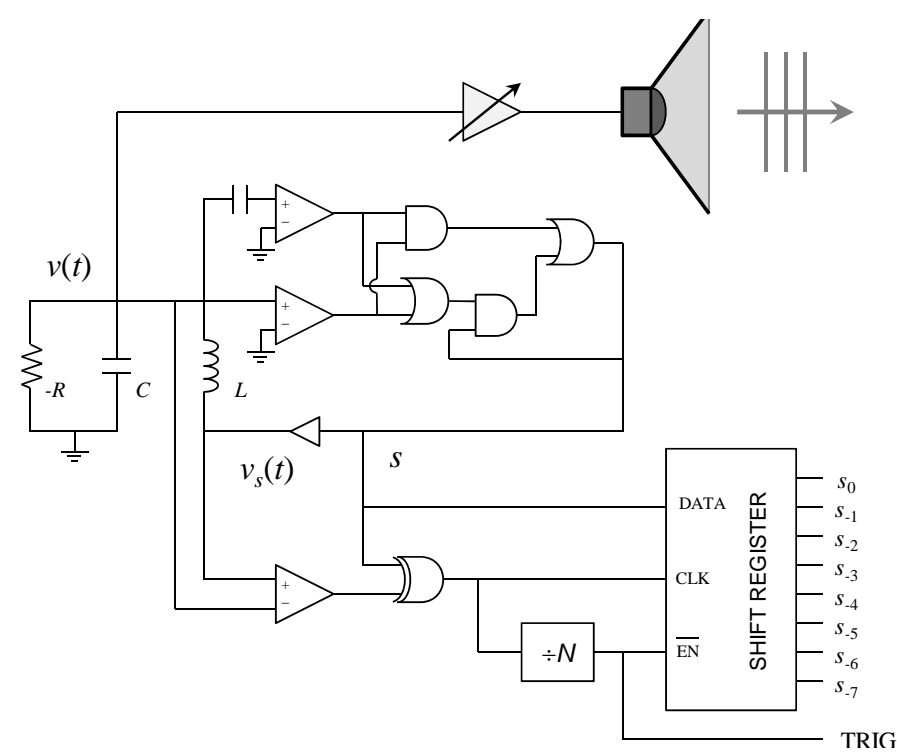

Figure 3. Acoustic transmitter incorporating the exactly solvable chaotic oscillator.
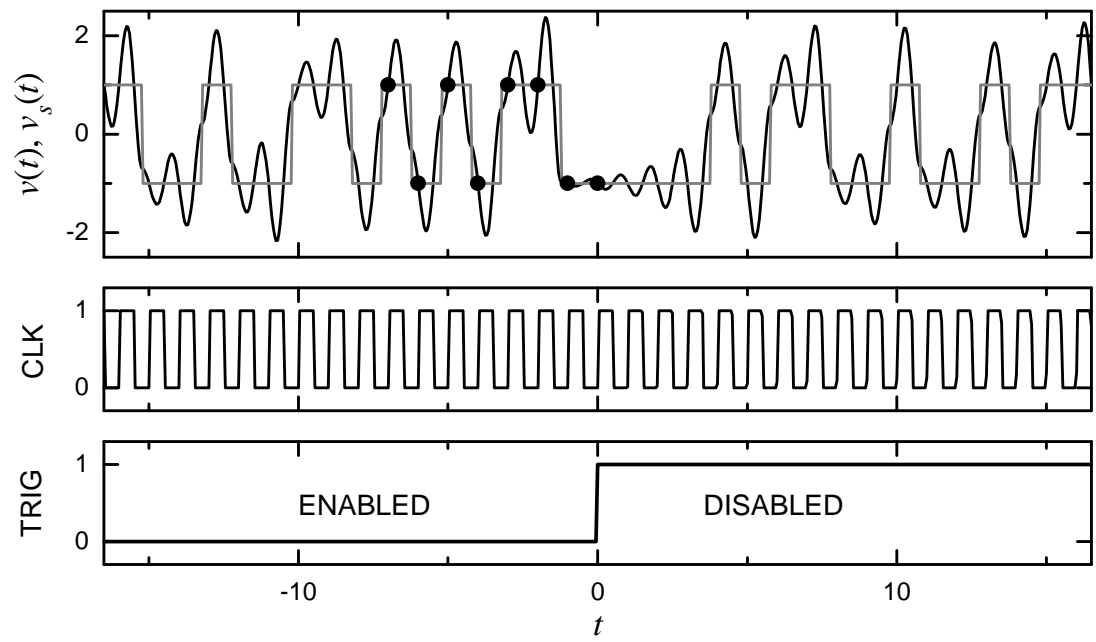

Figure 4. Typical transmitter waveforms, including the derived clock and trigger signals. Dots shown on the switching waveform indicate the most recent eight symbols stored in the shift register when disabled.

A simulated waveform and shift register content is shown in Fig. 4. The top plot shows the oscillator waveforms $v(t)$ and $v_{s}(t)$. The continuous waveform $v(t)$ is the transmitted waveform emitted by the speaker. The middle plot shows the clock signal that is extracted from the oscillator waveforms and defines the symbol timing. The bottom waveform shows the trigger signal derived by the divide-by- $N$ operation from the clock signal. The shift register is disabled by the low-tohigh transition of the trigger signal. The dots in the top plot show the most recent eight symbols captured and stored by the shift register when it is disabled by the trigger. These symbols define a reference waveform for the correlation receiver. 


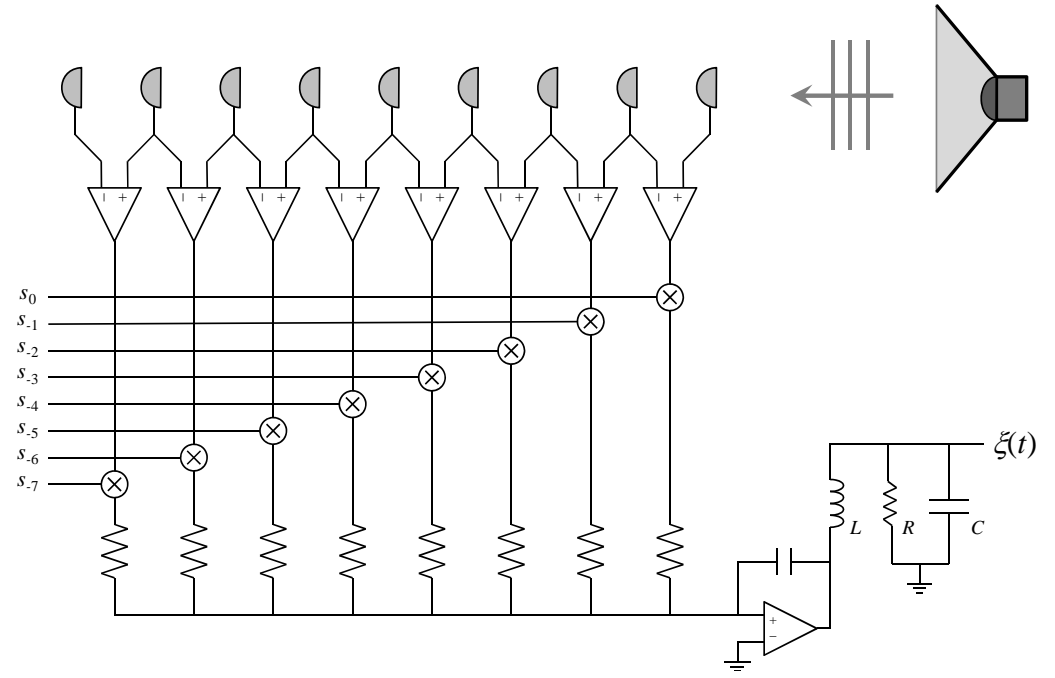

Figure 5. Acoustic correlation receiver that realizes a matched filter for the solvable oscillator.

\section{CORRELATION RECEIVER}

The second advantage in using an exactly solvable chaotic oscillator is the availability of a simple matched filter for the basis function [16]. This filter enables the construction of a simple correlation receiver for a chaotic waveform segment defined by truncated symbol sequence [13]. For the acoustic system, we implement a receiver using discrete circuit components and without requirement for a DSP.

The matched filter for a solvable chaotic waveform is

$$
\begin{array}{r}
\frac{d \eta}{d t}=\sum_{m=1-N}^{0} s_{m} \cdot\{\tilde{v}(t+1+m)-\tilde{v}(t+m)\} \\
\frac{d^{2} \xi}{d t^{2}}+2 \beta \frac{d \xi}{d t}+\left(\omega^{2}+\beta^{2}\right)(\xi-\eta)=0
\end{array}
$$

where $\tilde{v}$ is the received signal, $\eta$ is an intermediate state, and $\xi$ is the output of the matched filter [13]. The finite symbol sequence $s_{m}$ for $m=1-N, \ldots, 0$ defines the reference waveform for the filter. The function of the matched filter is mathematically equivalent to a correlation with the reference waveform. For a signal corrupted by additive white Gaussian noise (AWGN), the matched filter is the optimal linear receiver for detecting the signal [17].

Fig. 5 shows a schematic implementation of the matched filter for the acoustic ranging system. The received waveform impinges on a microphone array, shown at top. The spacing of the microphones in the array is chosen to realize the evenly spaced time delays in the first equation of the matched filter. Differential amplifiers between adjacent microphones provide the difference signal of successively lagged signals, which are multiplied by \pm 1 according to the symbols defining the reference waveform. The summed differences are integrated and drive the harmonic filter to generate the matched filter output.

\section{RANGING SYSTEM}

The transmitter and receiver were constructed and installed in an acoustically anechoic chamber for demonstration and test. The speaker was positioned at different ranges in front of the microphone array, as shown in Fig. 6. A handheld oscilloscope, triggered on the disable signal to the shift register, was used to monitor the receiver output. The transmitter and matched filter states were also connected to a computer for instrumentation and tuning purposes. 


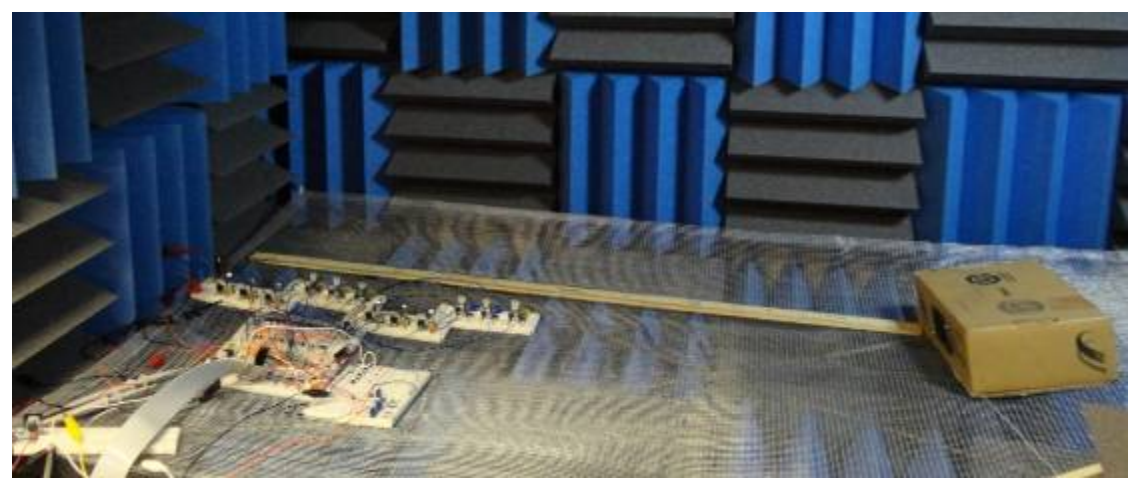

Figure 6. Acoustic ranging system using solvable chaos installed in an anechoic test chamber.

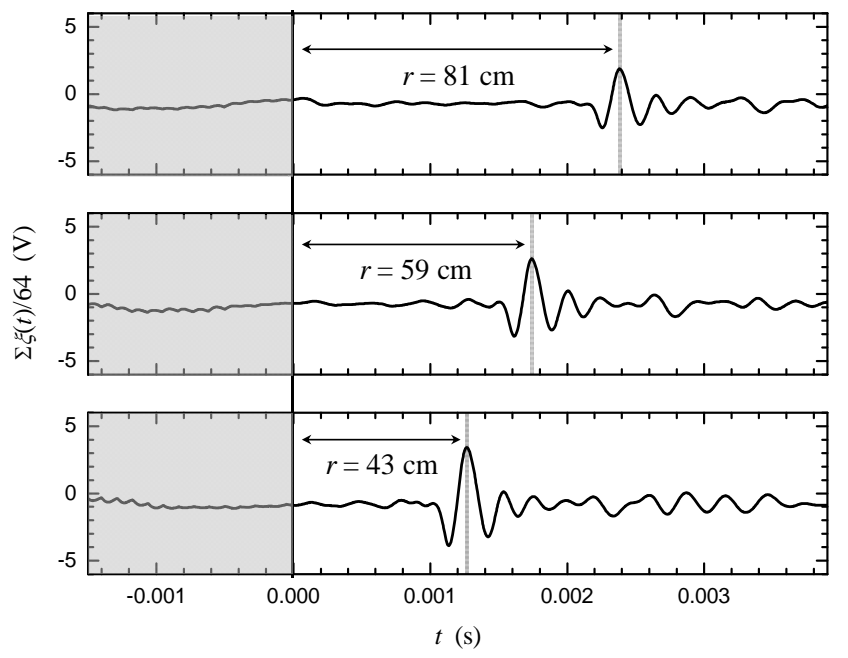

Figure 7. Receiver output for 64 averaged returns at three speaker positions.

In operation, a consistent spike in the matched filter output was evident at a delay corresponding to the distance from speaker to microphone array. In a single return for one instance of the reference waveform, this peak may be indistinguishable from background noise, intersymbol interference, or waveform sidelobes. However, the consistent peak emerges when multiple receiver outputs are averaged, which was conveniently provided by the oscilloscope. Typical outputs for 64 averaged returns are shown in Fig. 7, which shows the detection and correctly estimated range at three speaker positions.

\section{CONCLUSIONS}

We successfully demonstrated acoustic ranging using a simple and inexpensive experimental system by exploiting the properties of exactly solvable chaos. Notably, correlation receiver capability is achieved using analog hardware, and significant pulse compression is obtained without the sampling and storage requirements of a comparable random signal waveform. This successful demonstration enables the development of new, low-cost sonar and radar technologies using chaotic waveforms. 


\section{REFERENCES}

[1] K. M. Myneni, T. A. Barr, B. R. Reed, S. D. Pethel, and N. J. Corron, "High-precision ranging using a chaotic laser pulse train,” App. Phys. Lett. 78, 1496 (2001).

[2] B. C. Flores, E. A. Solis, and G. Thomas, "Assessment of chaos-based FM signals for range-Doppler imaging," IEE Proc.-Radar Sonar Navig. 150, 313 (2003).

[3] F.-Y. Lin and J.-M. Liu, "Ambiguity functions of laser-based chaotic radar," IEEE J. Quantum Elec. 40, 1732 (2004).

[4] F.-Y. Lin and J.-M. Liu, “Chaotic radar using nonlinear laser dynamics,” IEEE J. Quantum Elec. 40, 815 (2004).

[5] V. Venkatasubramanian and H. Leung, "A novel chaos-based high-resolution imaging technique and its application to through-the-wall imaging,” IEEE Signal Proc. Lett. 12, 528 (2005).

[6] T. L. Carroll, "Chaotic system for self-synchronizing Doppler measurement," Chaos 15, 013109 (2005).

[7] T. L. Carroll, “Optimizing chaos-based signals for complex targets,” Chaos 17, 033103 (2007).

[8] Z. Liu, X. Zhu, W. Hu, and F. Jiang, "Principles of chaotic signal radar,” Int. J. Bifurcation Chaos Appl. Sci. Eng. 17, 1735 (2007).

[9] S. Qiao, Z. G. Shi, K. S. Chen, W. Z. Cui, W. Ma, T. Jiang, and L. X. Ran, "A new architecture of UWB radar utilizing microwave chaotic signals and chaos synchronization,” Prog. Electromagnetics Res. 75, 225 (2007).

[10] Z. G. Shi, S. Qiao, K. S. Chen, W. Z. Cui, W. Ma, T. Jiang, and L. X. Ran, "Ambiguity functions of direct chaotic radar employing microwave chaotic Colpitts oscillator,” Prog. Electromagnetics Res. 77, 1 (2007).

[11] T. L. Carroll, “Adaptive chaotic maps for identification of complex targets," IET Radar Sonar Navig. 2, 256 (2008).

[12] T. Jiang, S. Qiao, Z. Shi, L. Peng, J. Huangfu, W. Z. Cui, W. Ma, and L. X. Ran, "Simulation and experimental evaluation of the radar signal performance of chaotic signals generated from a microwave Colpitts oscillator," Prog. Electromagnetics Res. 90, 15 (2009).

[13] J. N. Blakely and N. J. Corron, "Concept for low cost chaos radar using coherent reception,” Proc. SPIE 8021, 80211H (2011).

[14] N. J. Corron, J. N. Blakely, “Chaos for Communication and Radar,” Proc. NOLTA, 322 (2011).

[15] T. Tsubone and T. Saito, "Stabilizing and destabilizing control for a piecewise-linear circuit," IEEE Trans. Circuits Syst. I 45, 172 (1998).

[16] N. J. Corron, J. N. Blakely, and M. T. Stahl, “A matched filter for chaos,” Chaos 20, 023123 (2010).

[17] G. L. Turin, “An introduction to matched filters," IRE T. Inform. Theor. 6, 311 (1960). 


\section{Demonstration of detection and ranging using solvable chaos}

Ned J. Corron a, Mark T. Stahlb, Jonathan N. Blakelya

a U. S. Army AMRDEC, Redstone Arsenal, Alabama b NASA MSFC, Redstone Arsenal, Alabama 


\section{Chaotic Waveforms}

- Wide bandwidth

- high range resolution

- low probability of detection

- anti-jamming

- Non-repeating

- unambiguous ranging

- multi-user

- Auto-synchronizing

- power combining

- beam steering

- Simple devices

- low cost

- efficient

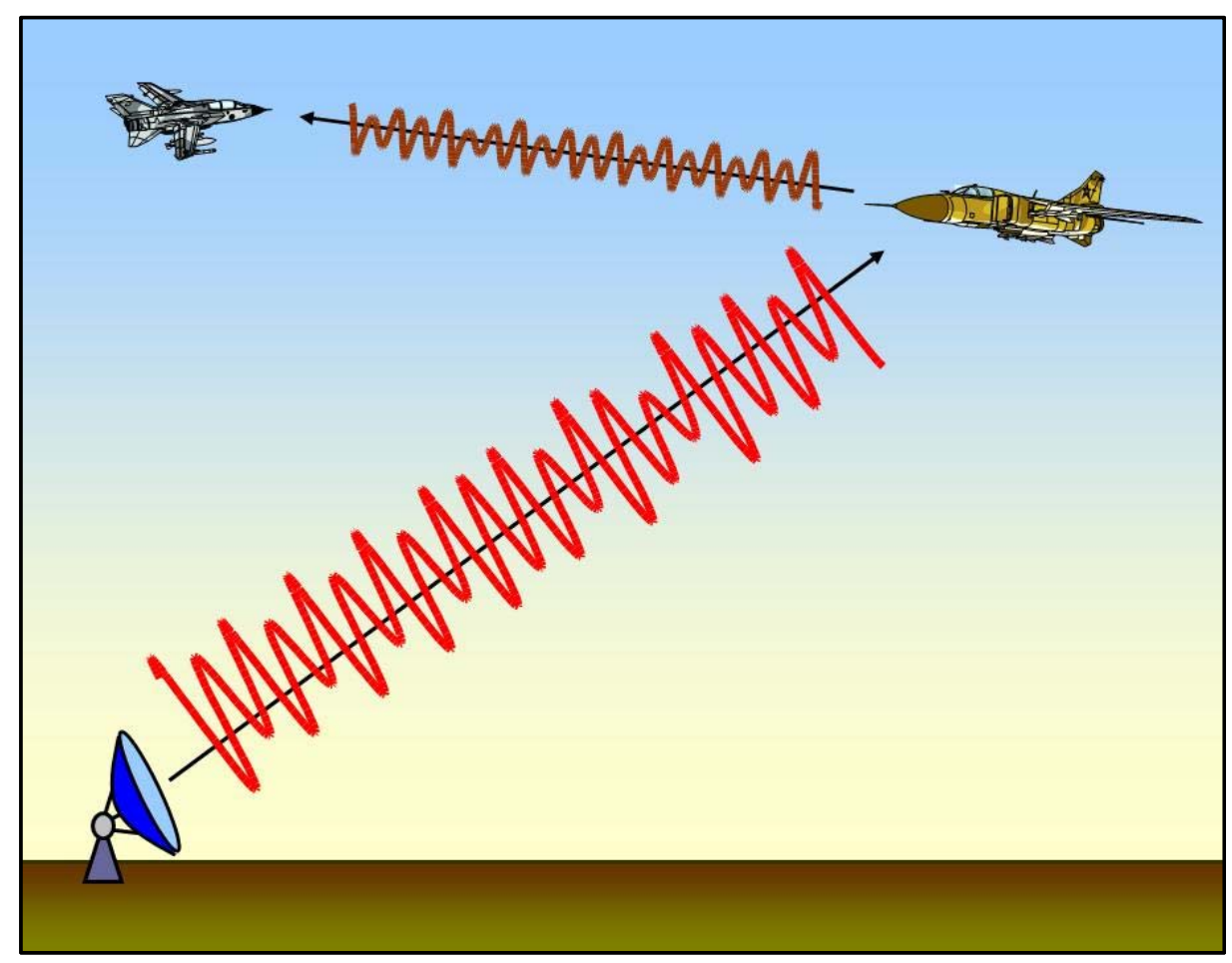

Chaotic Waveforms Appear Well Suited for Radar Applications... 


\section{Noise Radar}

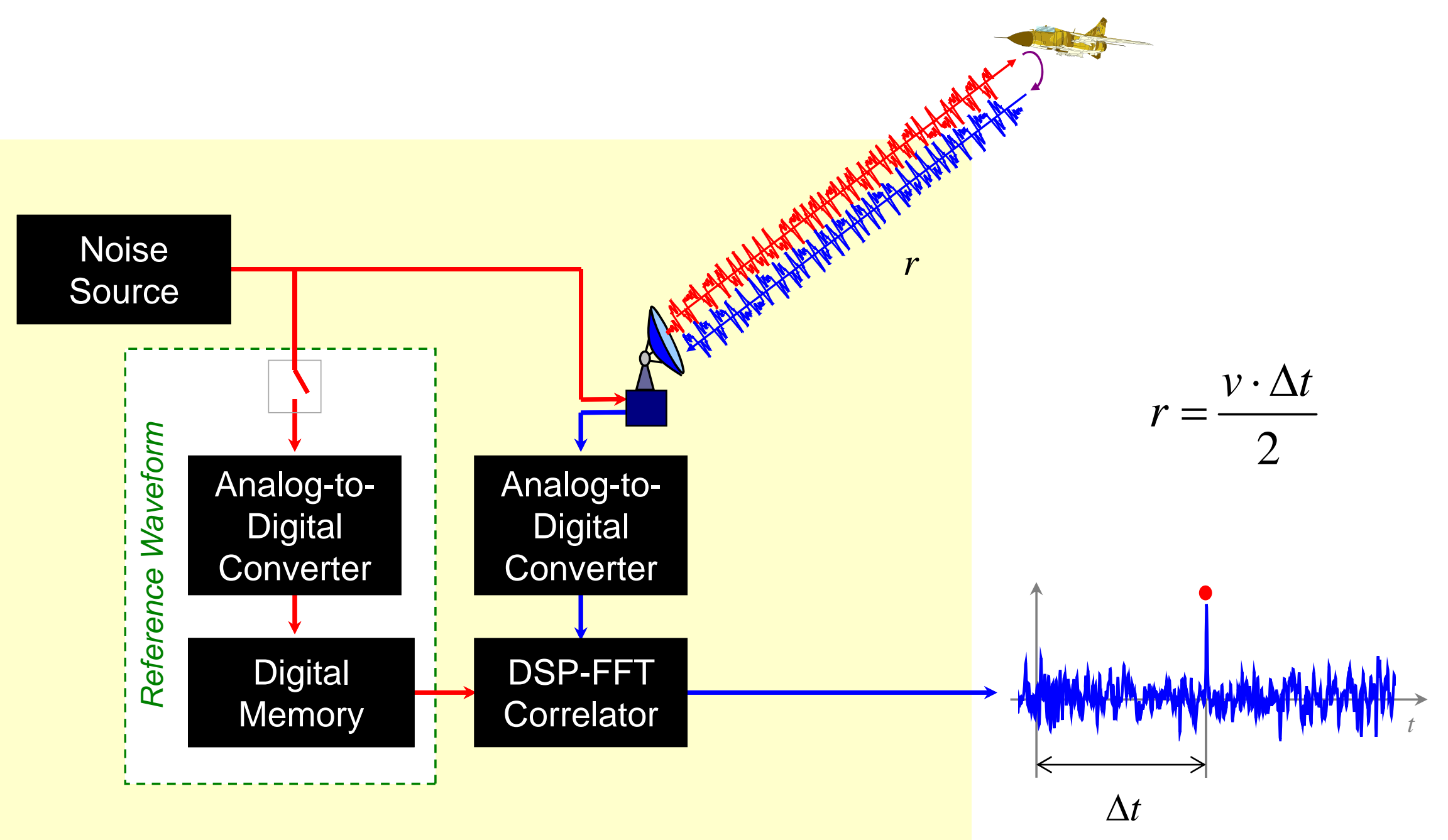




\section{Chaos Noise Radar}

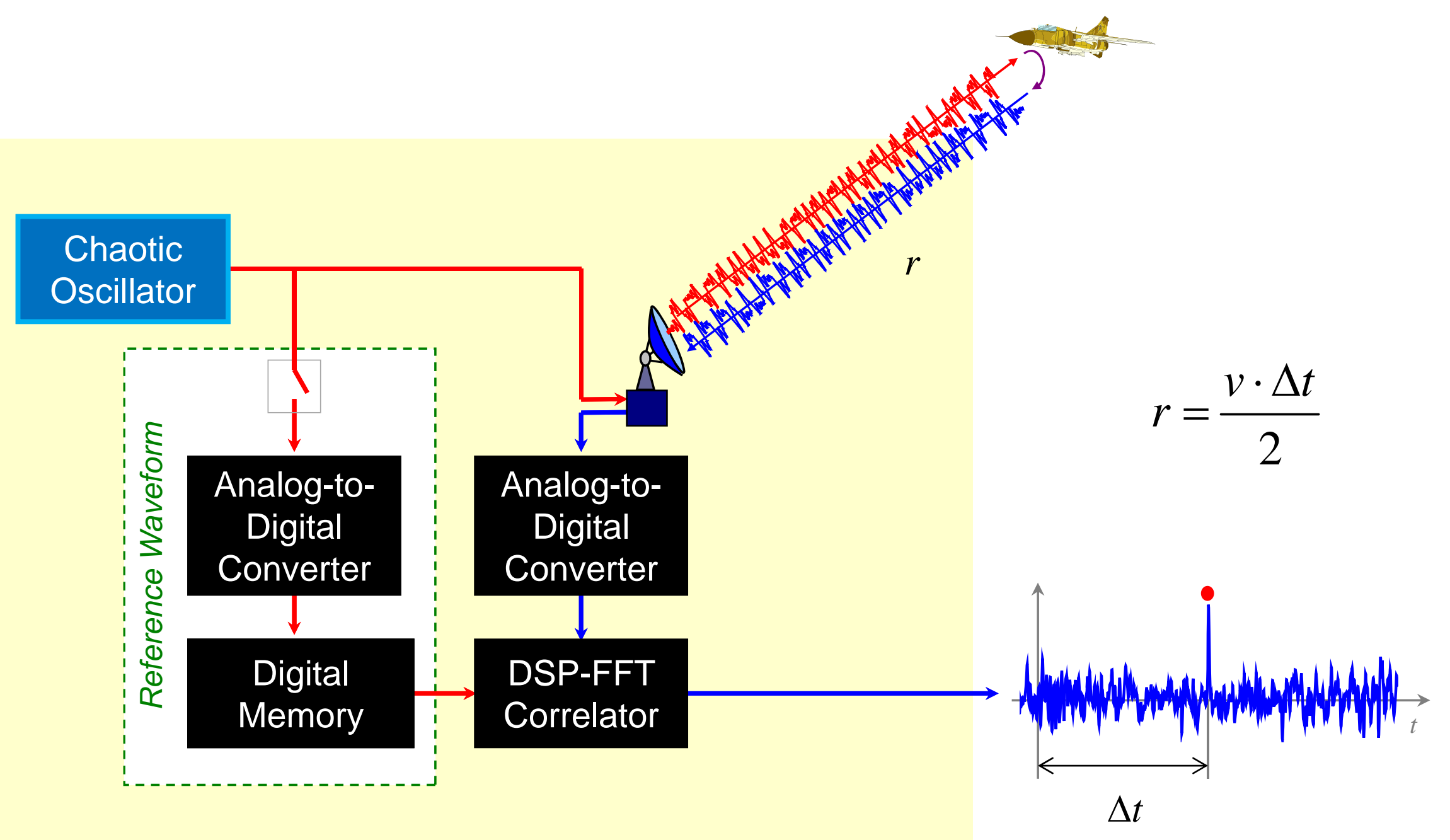




\section{Designing a Waveform}

- Random bit sequence

$$
\left\{\ldots, s_{-2}, s_{-1}, s_{0}, s_{1}, s_{2}, \ldots\right\} \quad s_{m}= \pm 1
$$

- Encoded waveform

$$
u(t)=\sum_{m=-\infty}^{\infty} s_{m} \cdot \rho(t-m)
$$

- Basis function

Example: Binary Phase Shift Keying (BPSK)

$$
\rho(t)=? ? ?
$$




\section{Receiver Constraints}

- Want correlation performance

- Cannot afford digital processing

-What if we use a very simple, analog matched filter?
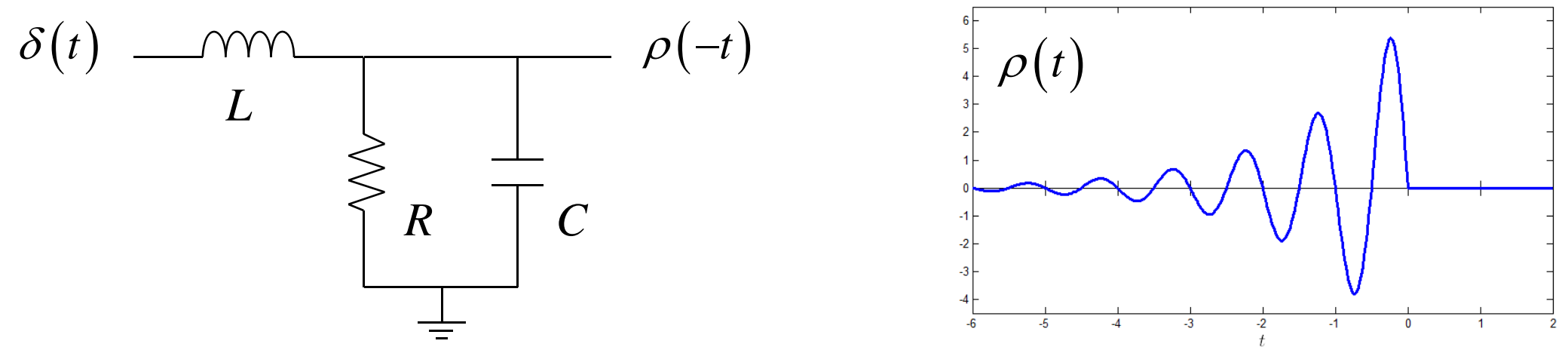

$$
M_{1} \circ \delta(t)=\rho(-t) \quad \sum \rho(t)=\left\{\begin{array}{cc}
-\frac{\omega^{2}+\beta^{2}}{\omega} \cdot \sin (\omega t) \cdot 2^{t}, & t<0 \\
0, & t \geq 0
\end{array}\right.
$$

Note: RLC values selected to meet "tuning" requirements: $\quad \beta=\ln 2, \quad \omega=2 \pi$ 


\section{Transformed Basis Function}
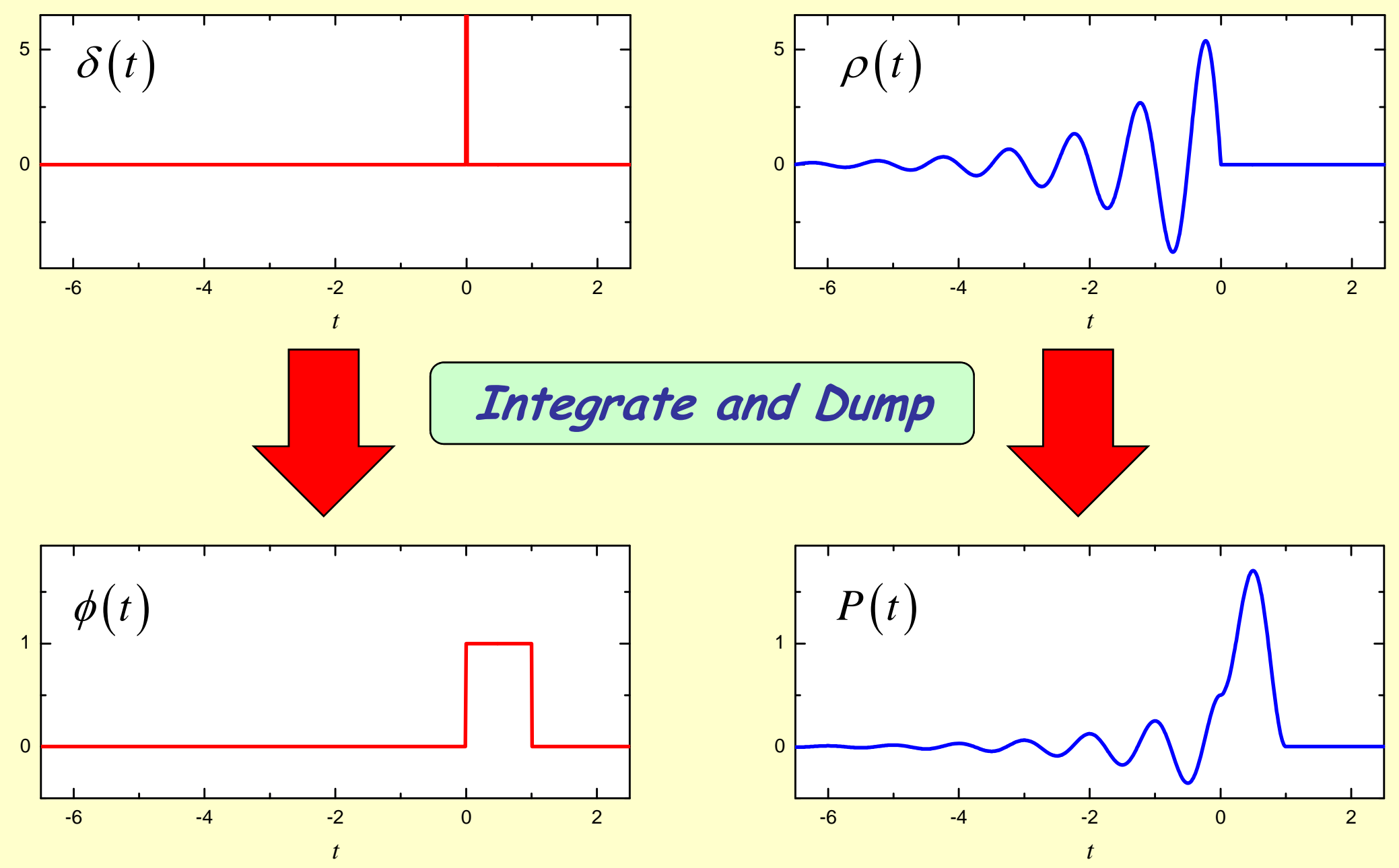


\section{Linear Synthesis:}

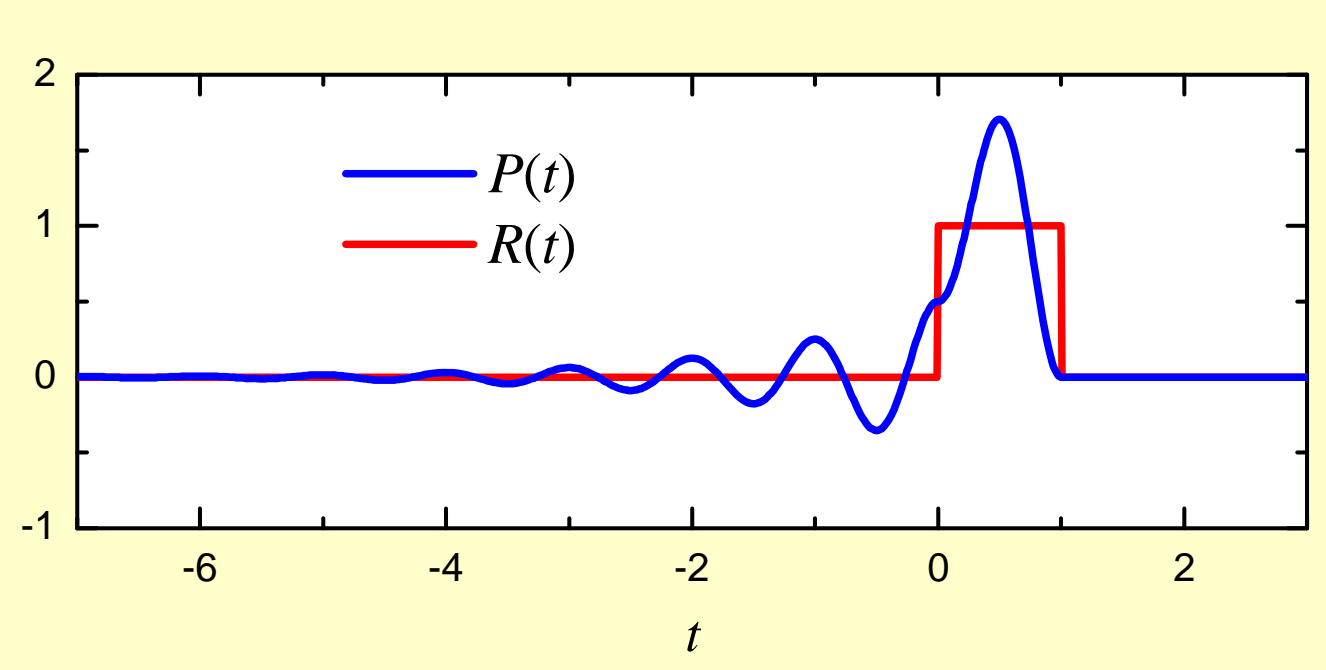

$$
\begin{gathered}
v(t)=\sum_{m=-\infty}^{\infty} s_{m} \cdot P(t-m) \\
v_{s}(t)=\sum_{m=-\infty}^{\infty} s_{m} \cdot R(t-m) \\
s_{m}= \pm 1
\end{gathered}
$$

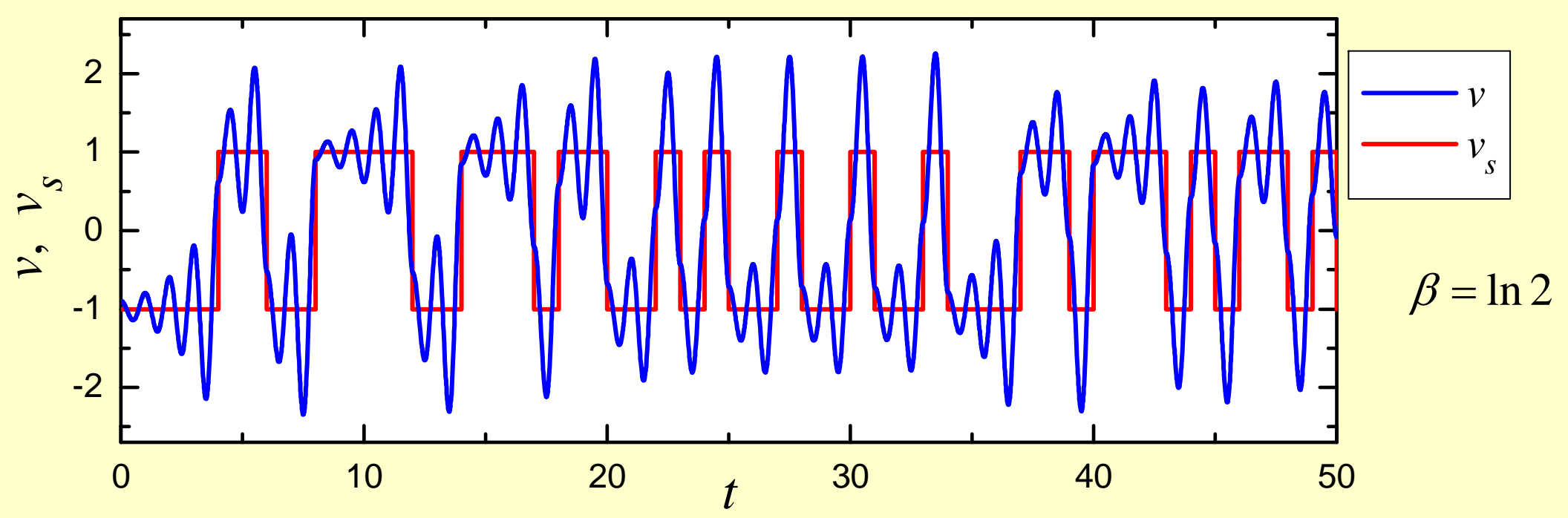




\section{Chaotic Oscillator}

- Hybrid oscillator circuit (analog and digital components):

$s=\{T, F\}$

$\Uparrow$

$v_{S}= \pm 1 \mathrm{~V}$
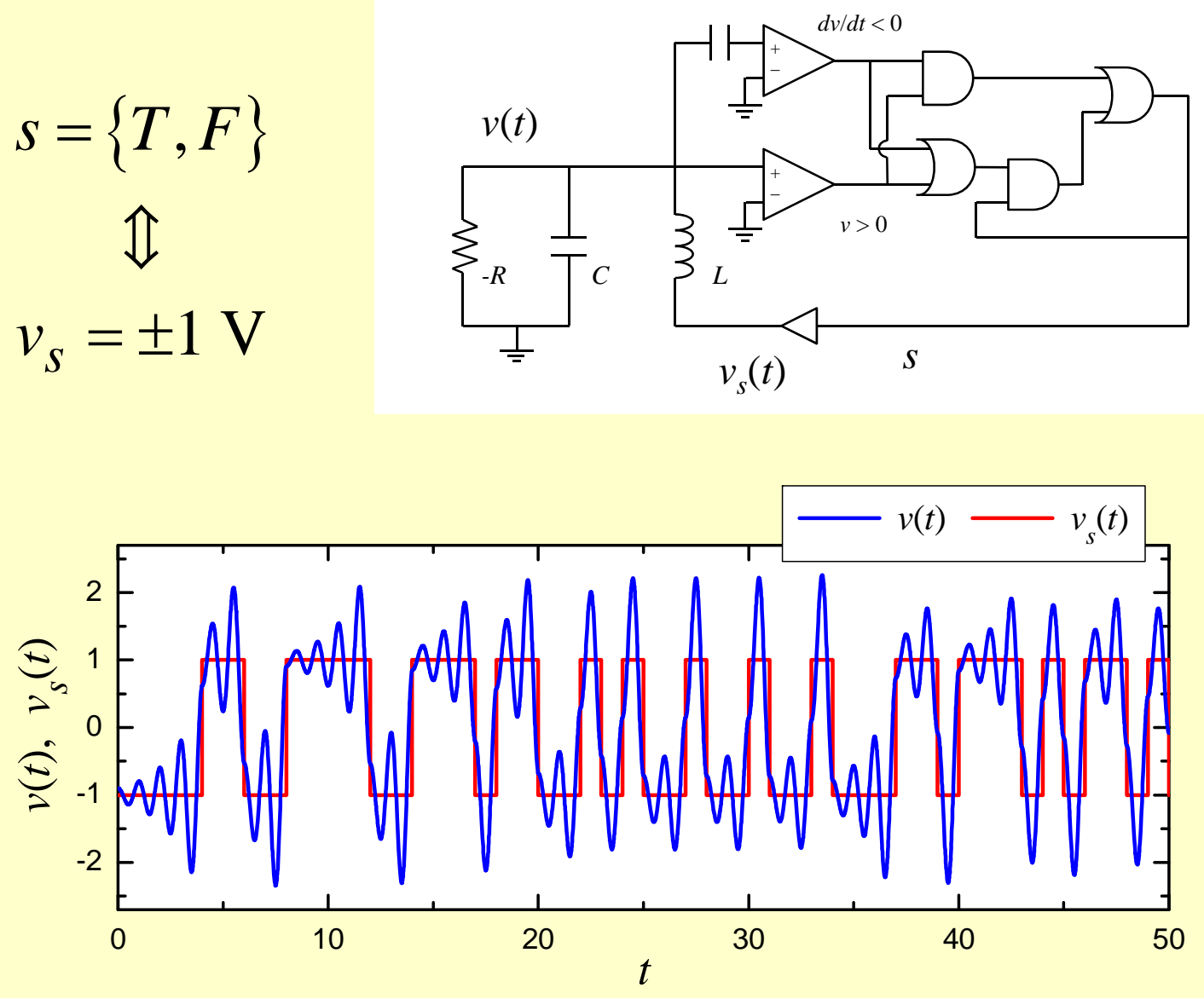
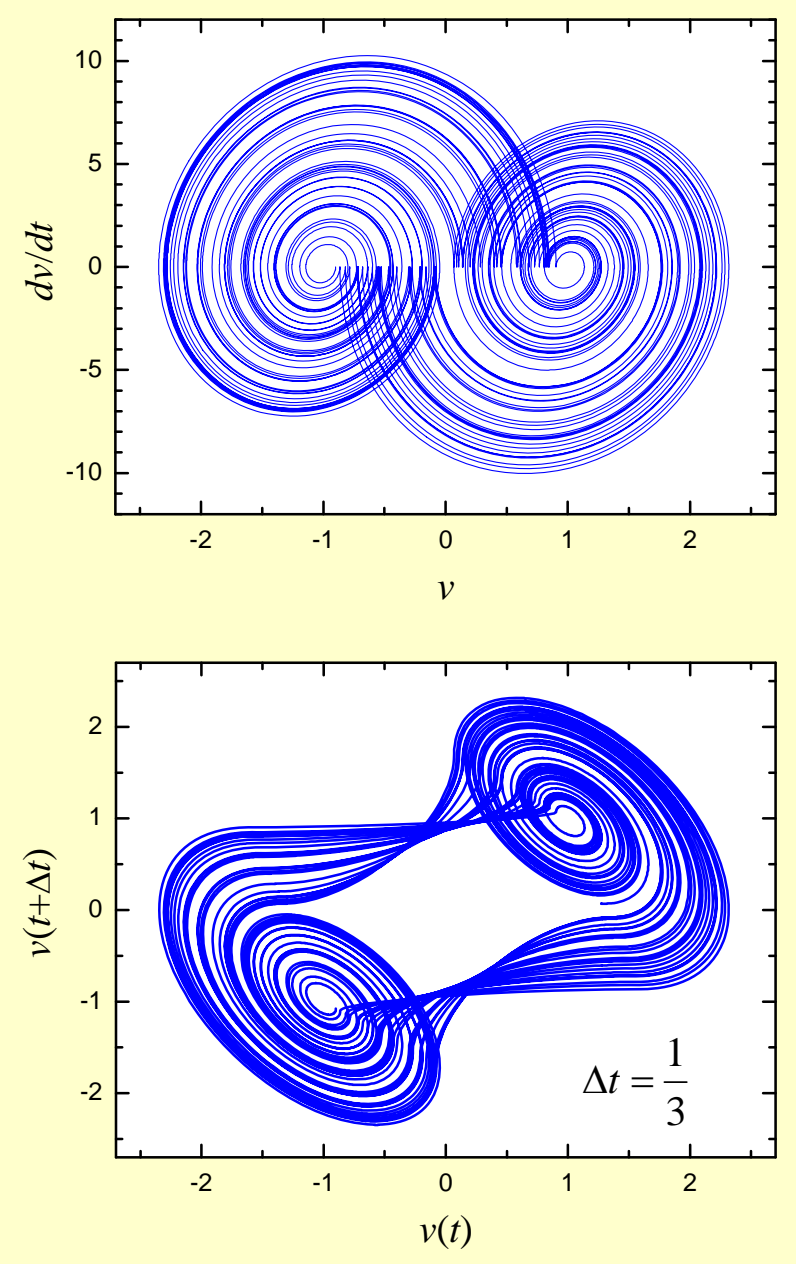


\section{Transmitter}

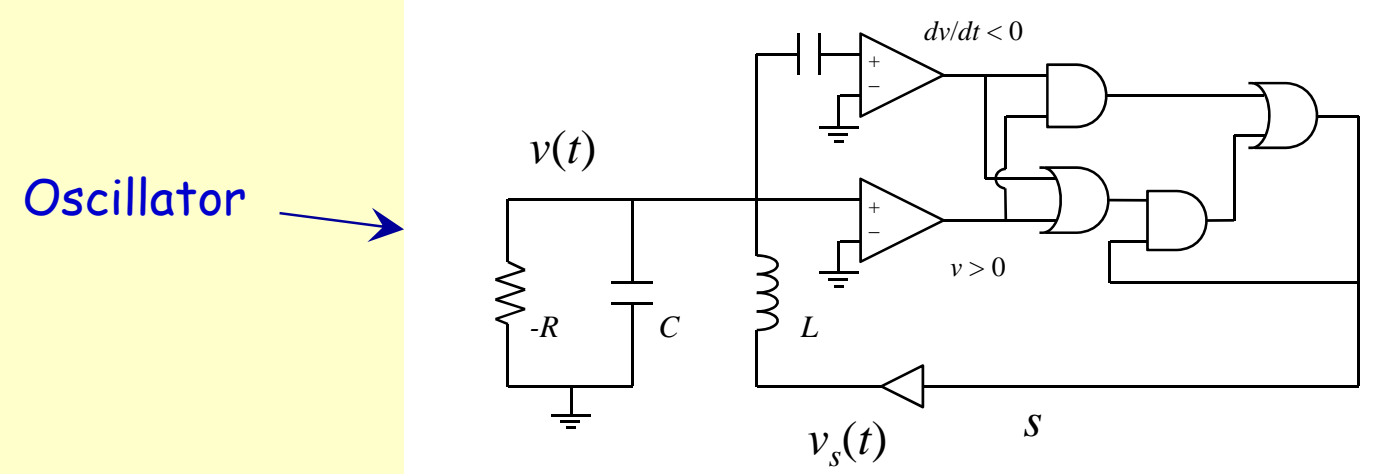




\section{Transmitter}

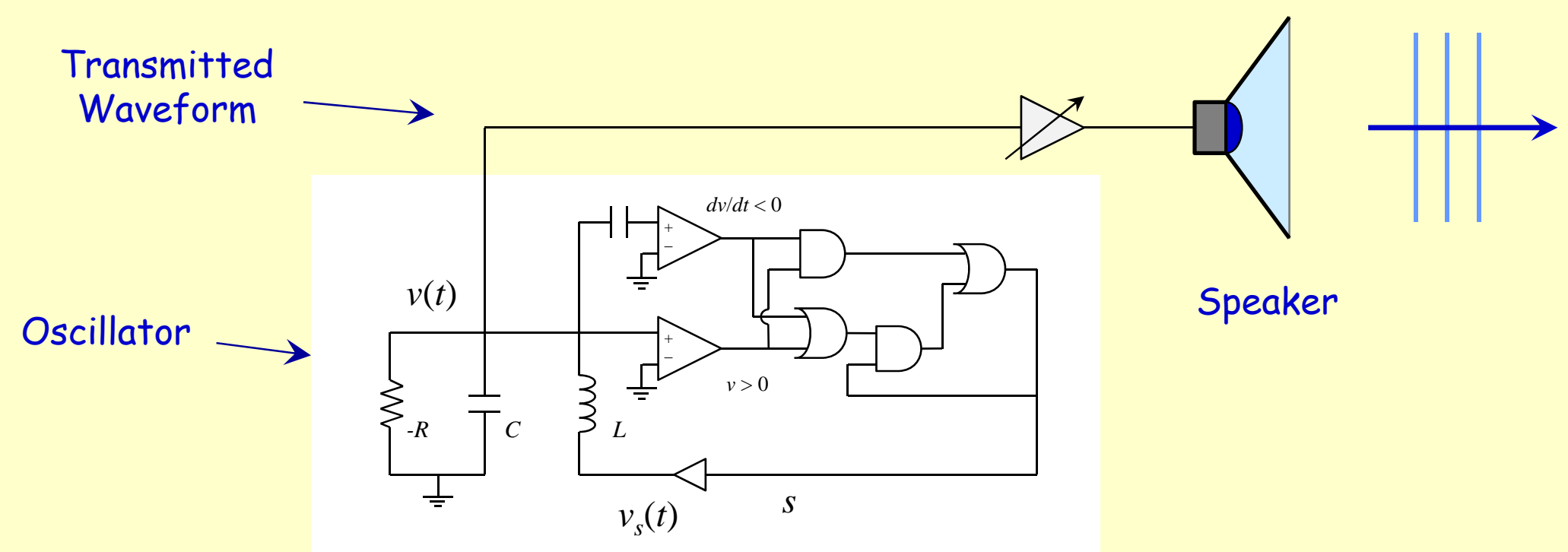




\section{Transmitter}

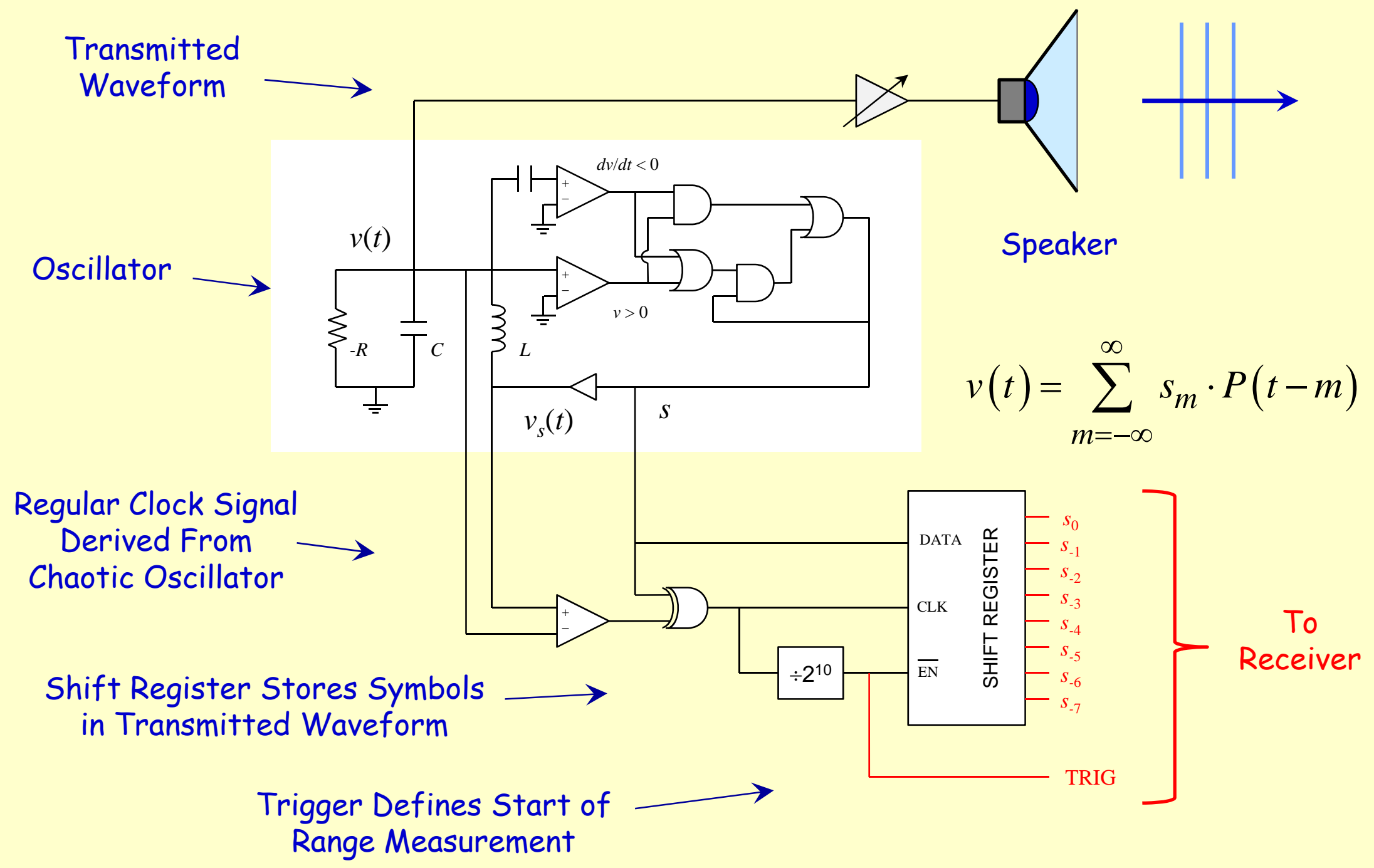




\section{Shift Register}
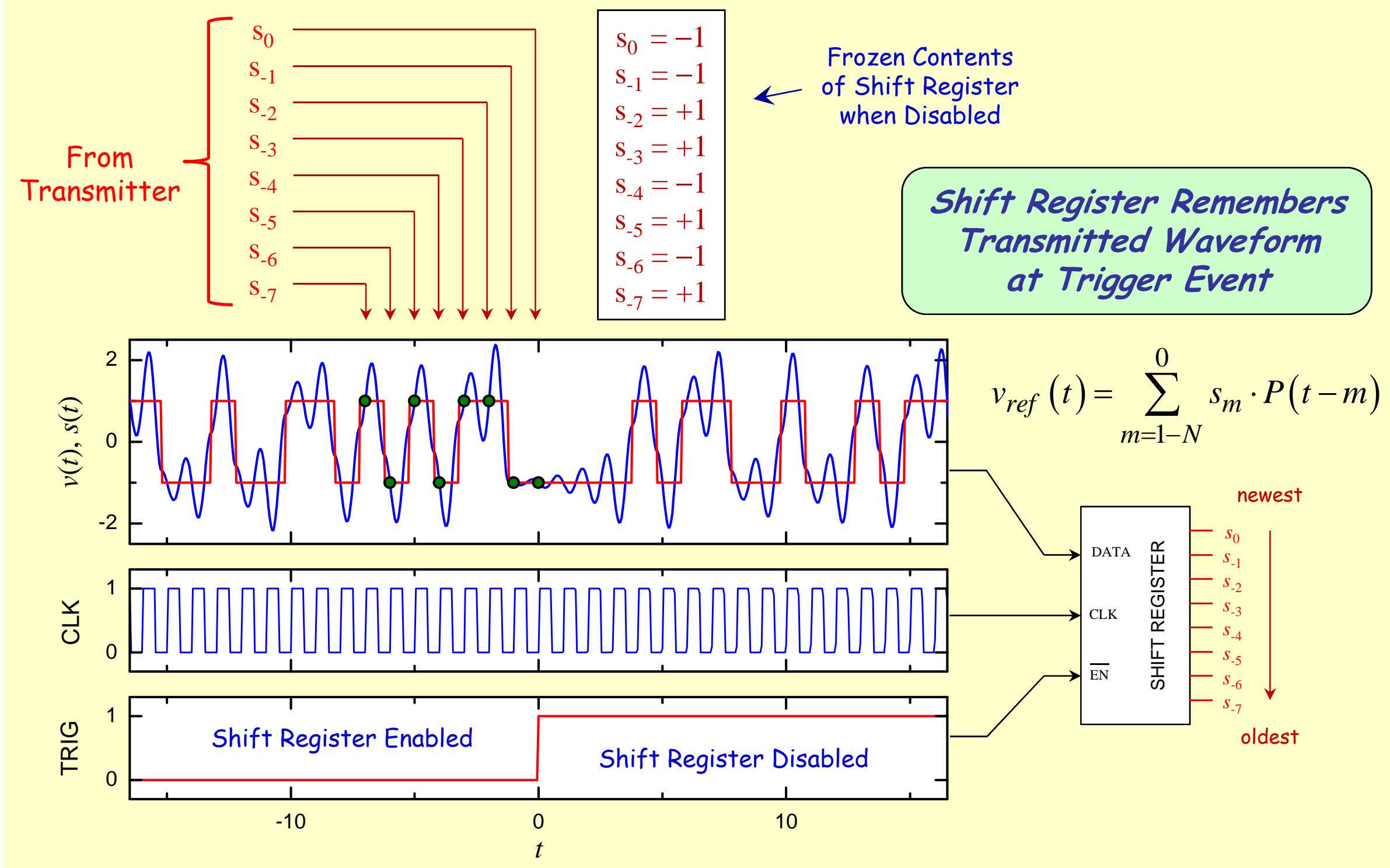


\section{Linear Convolution Waveform}

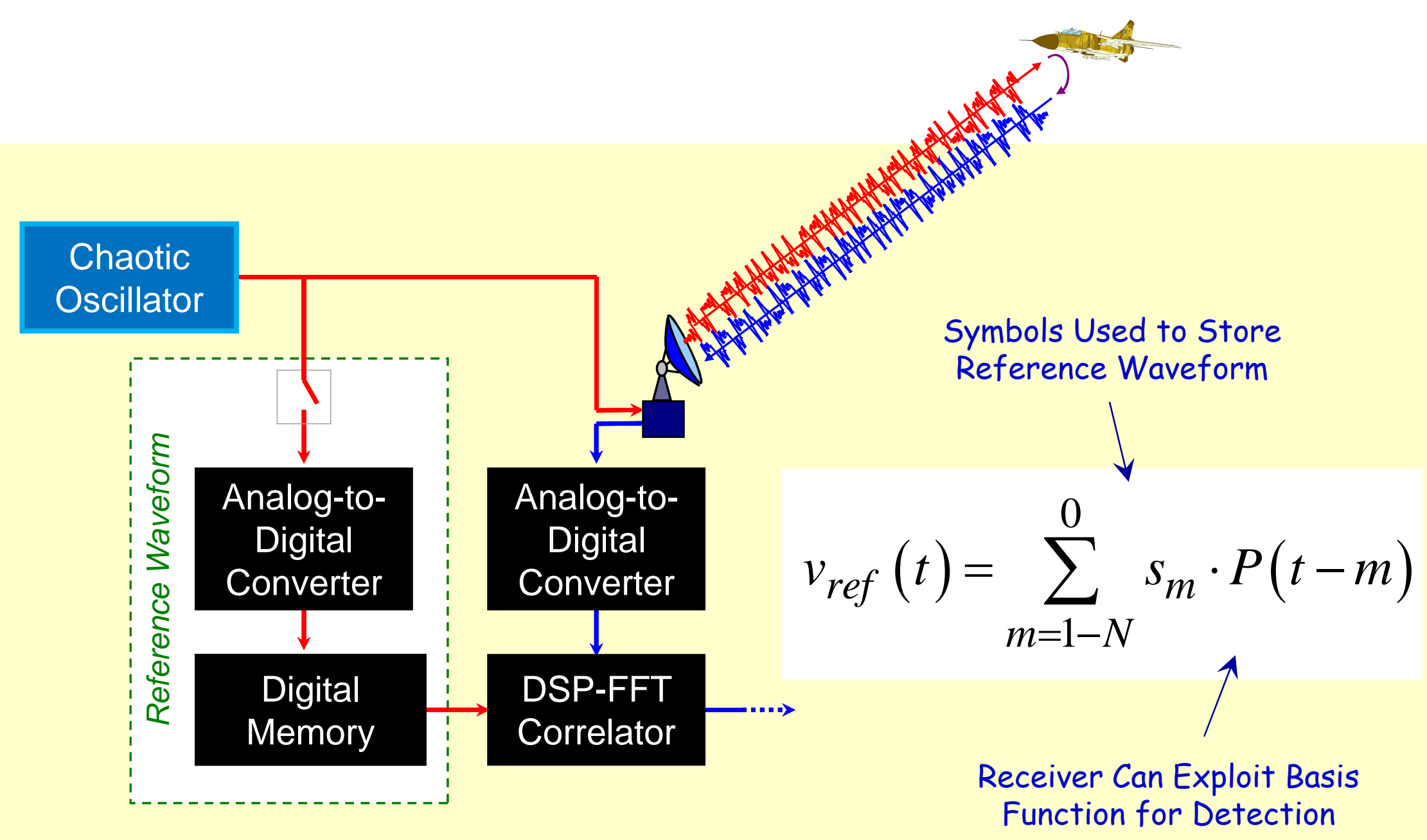




\section{Linear Convolution Waveform}

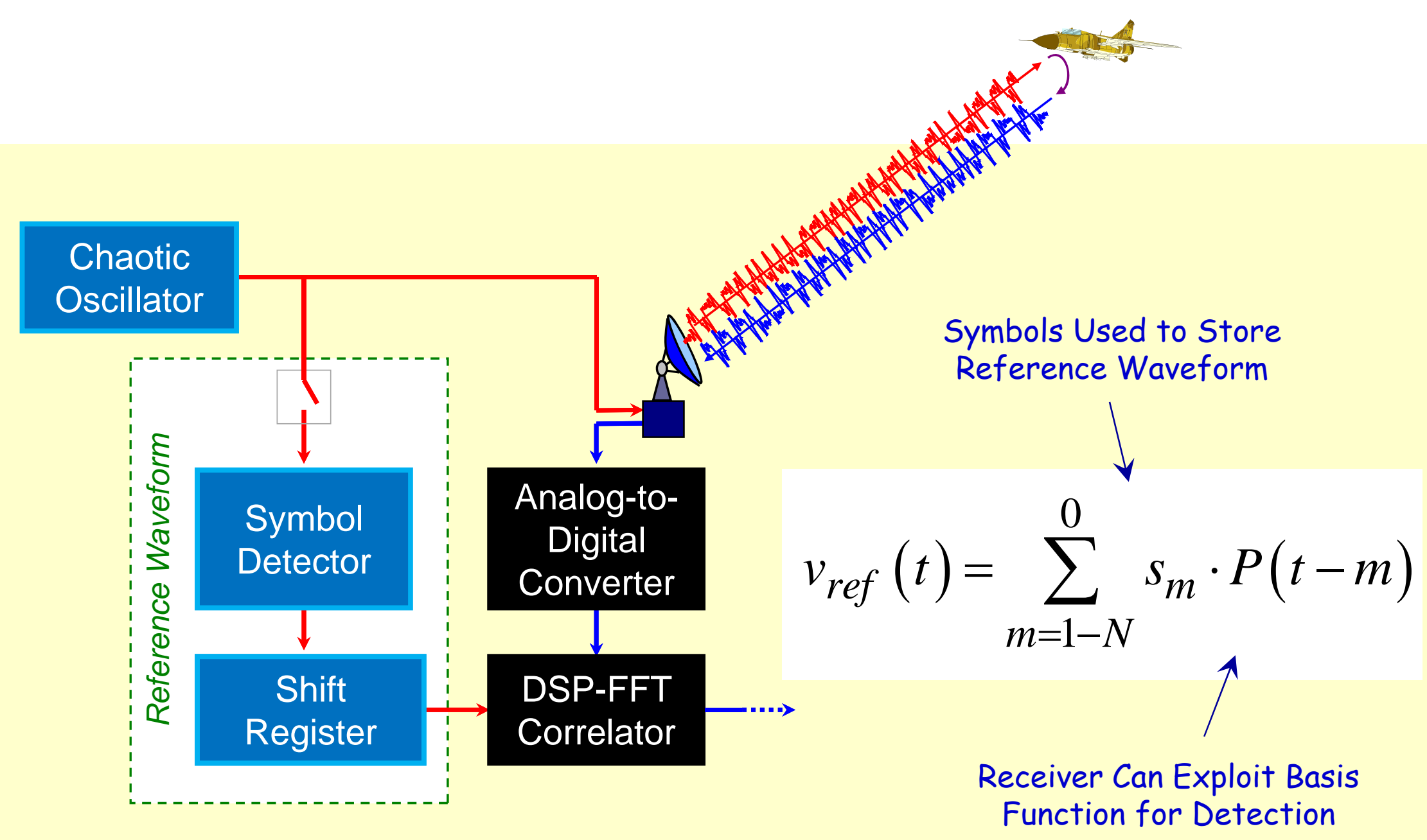




\section{Matched Filter}

$$
v_{\text {ref }}(t)=P(t)
$$

\section{Matched Filter}

Integrate and Dump:

$$
\frac{d \eta}{d t}=\tilde{v}(t+1)-\tilde{v}(t)
$$

Harmonic Filter:

$$
\begin{array}{r}
\frac{d^{2} \xi}{d t^{2}}+2 \beta \frac{d \xi}{d t}+\left(\omega^{2}+\beta^{2}\right) \xi \\
=\left(\omega^{2}+\beta^{2}\right) \cdot \eta(t)
\end{array}
$$

Microphone

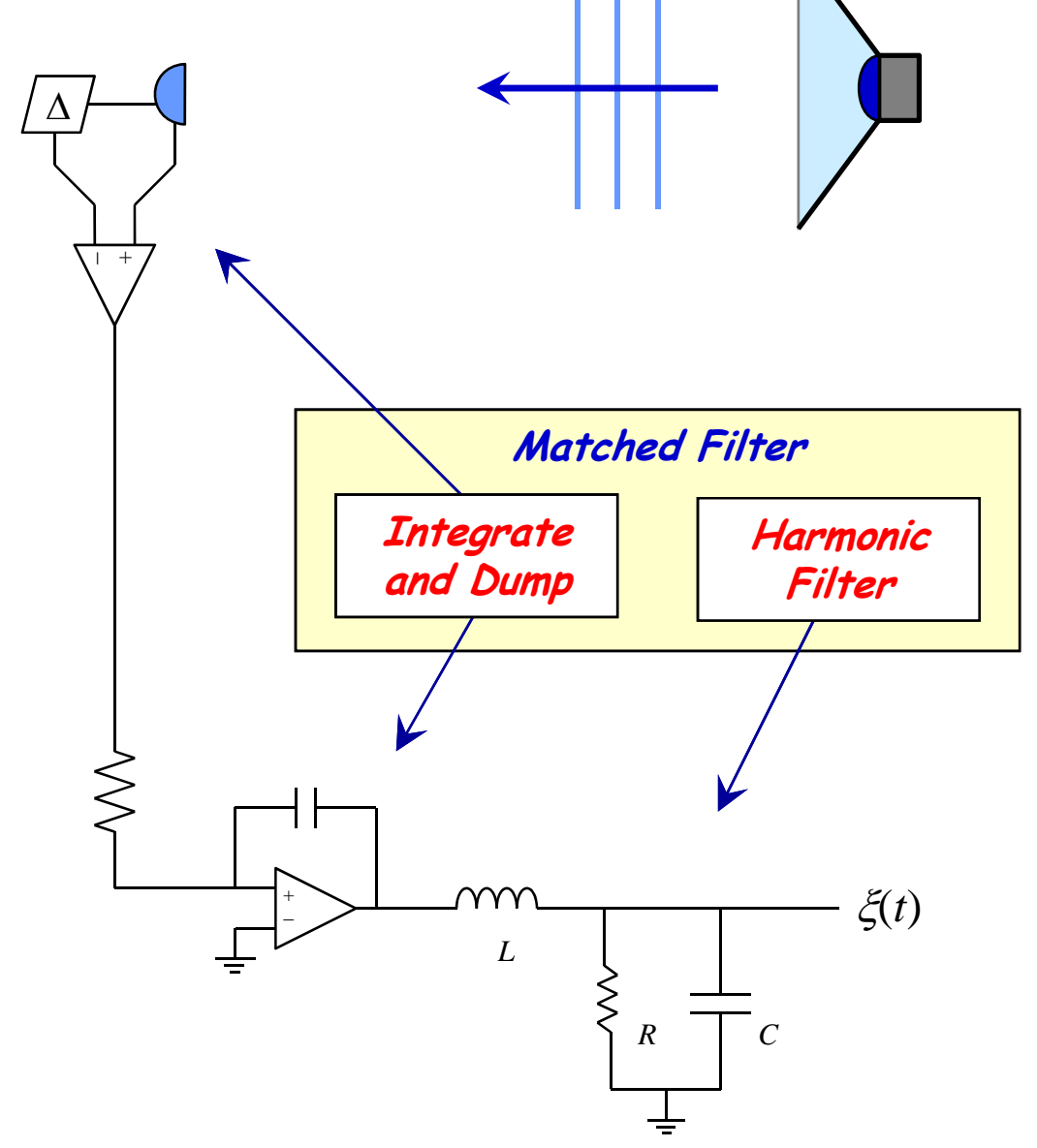




\section{Matched Filter}

$$
v_{\text {ref }}(t)=P(t)
$$

\section{Matched Filter}

Integrate and Dump:

$$
\frac{d \eta}{d t}=\tilde{v}(t+1)-\tilde{v}(t)
$$

Harmonic Filter:

$$
\begin{array}{r}
\frac{d^{2} \xi}{d t^{2}}+2 \beta \frac{d \xi}{d t}+\left(\omega^{2}+\beta^{2}\right) \xi \\
=\left(\omega^{2}+\beta^{2}\right) \cdot \eta(t)
\end{array}
$$

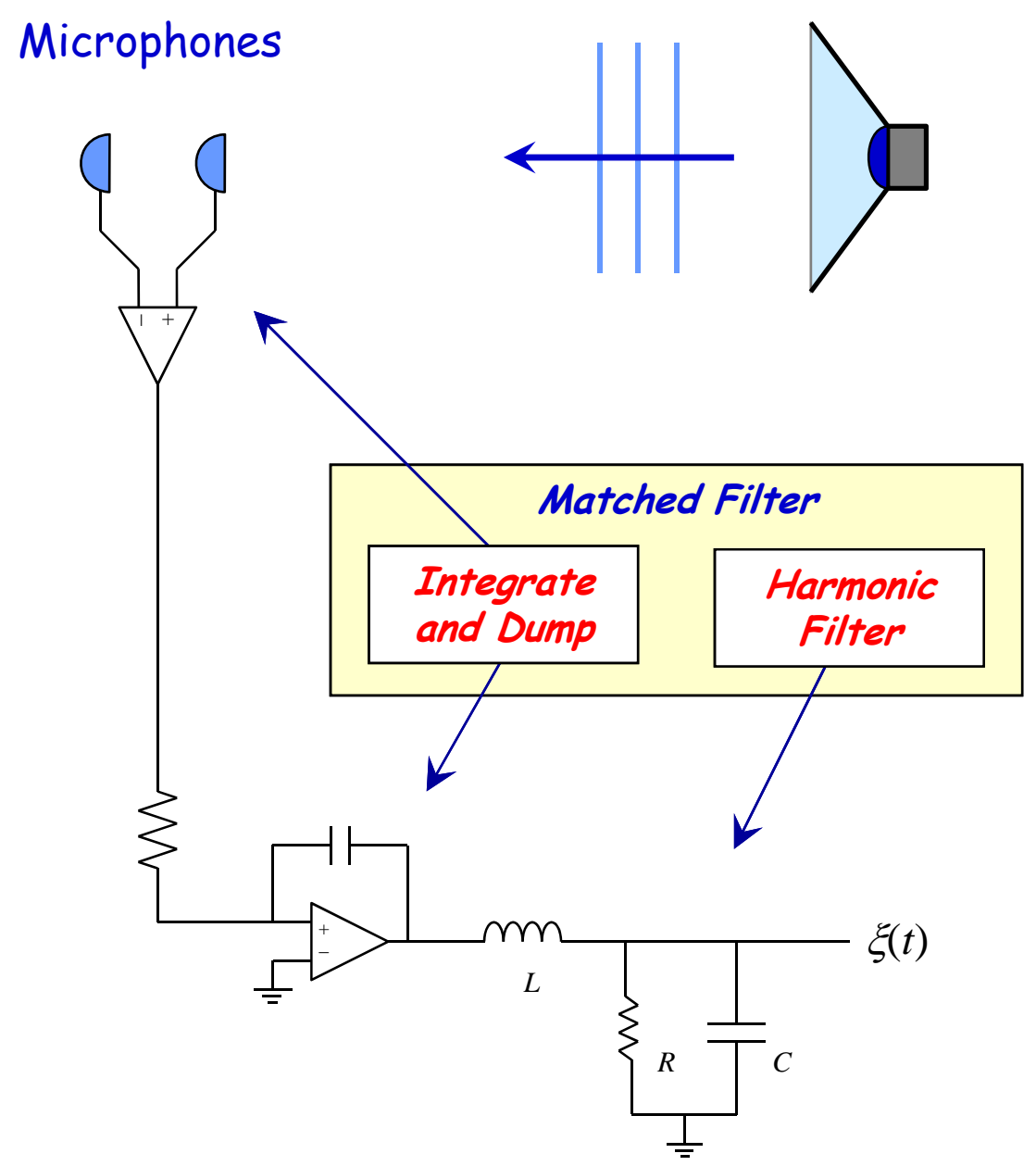




\section{Correlation Receiver}

$$
v_{r e f}(t)=\sum_{m=0}^{0} s_{m} \cdot P(t-m)
$$

Microphones

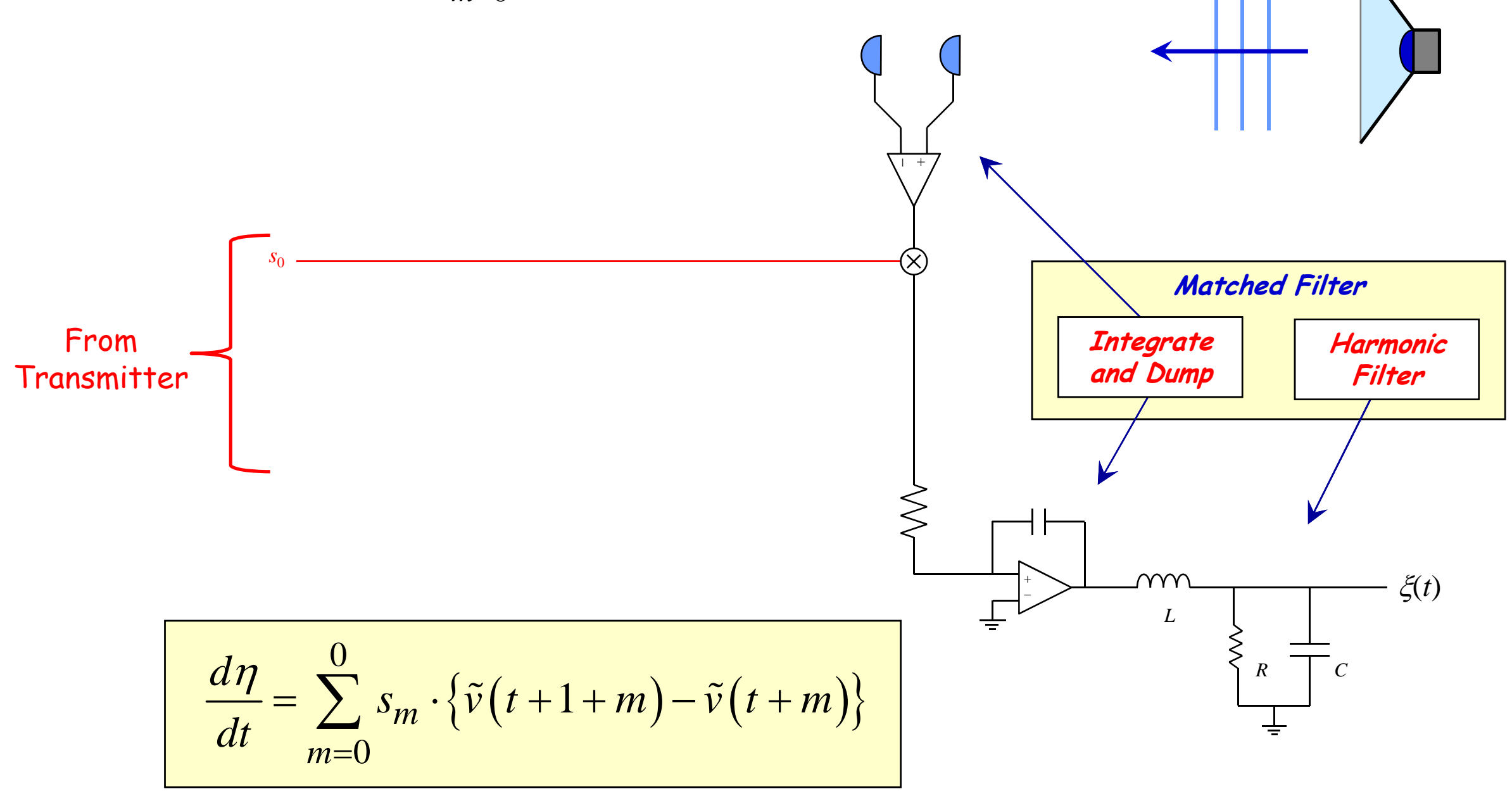




\section{Correlation Receiver}

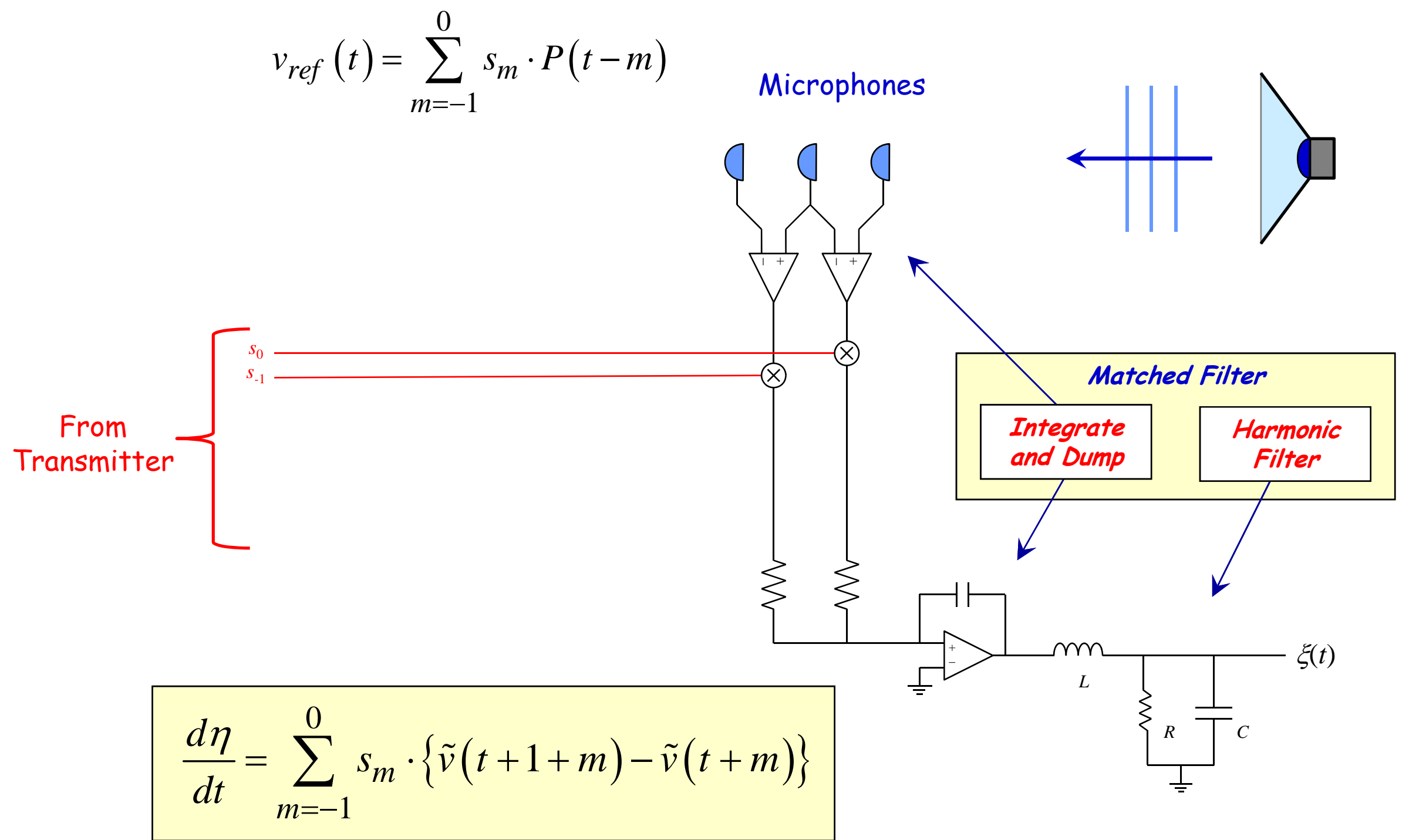




\section{Correlation Receiver}

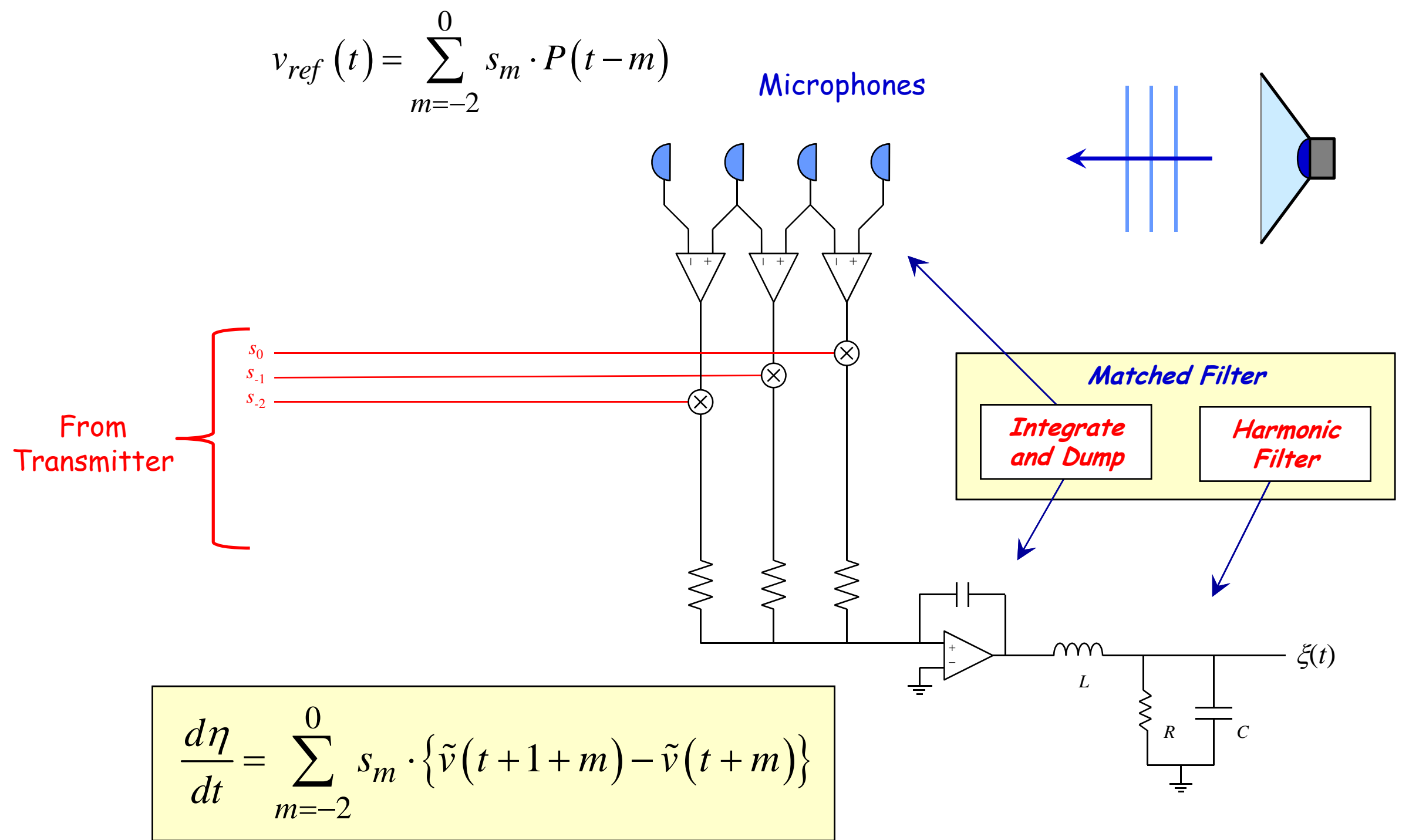




\section{Correlation Receiver}

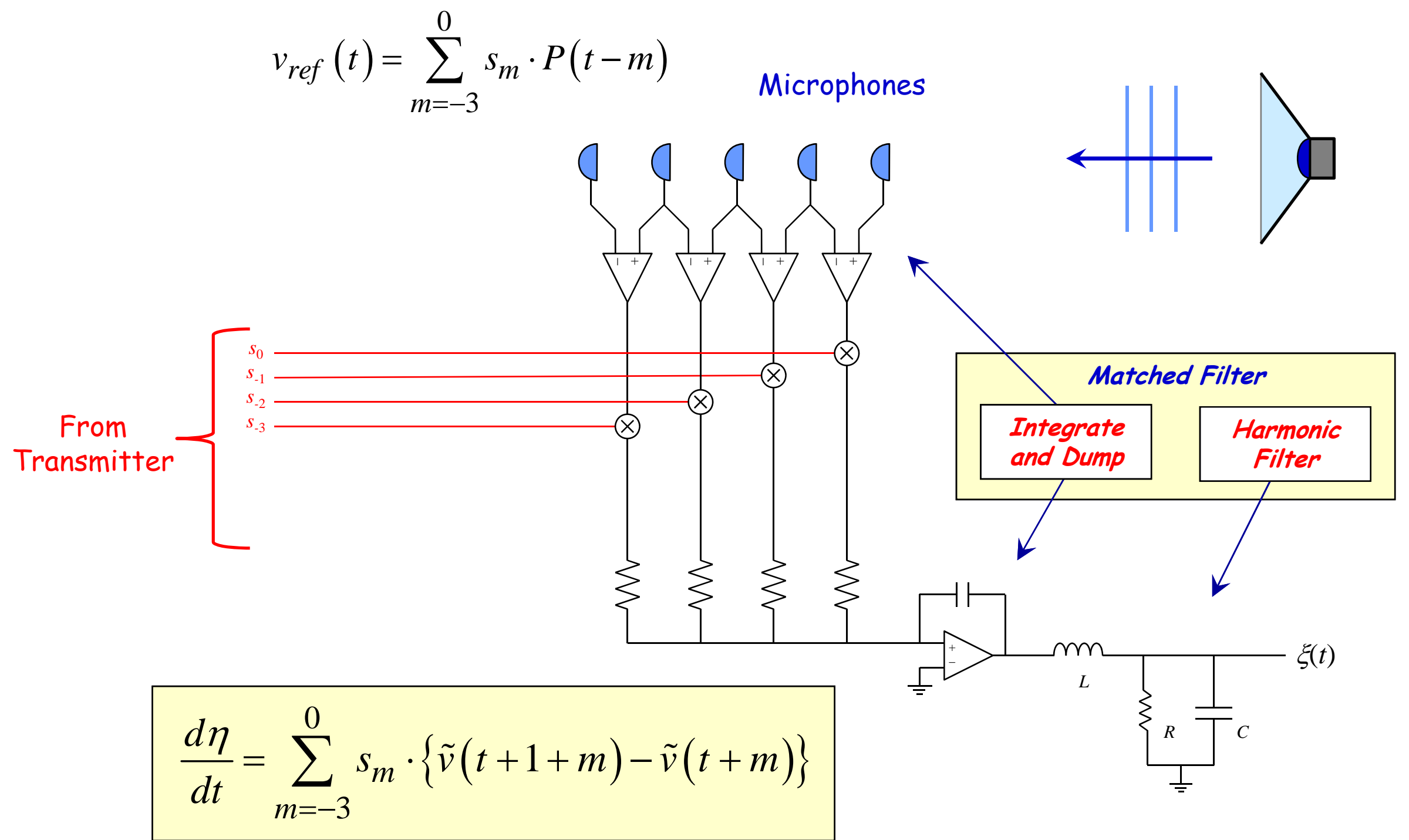




\section{Correlation Receiver}

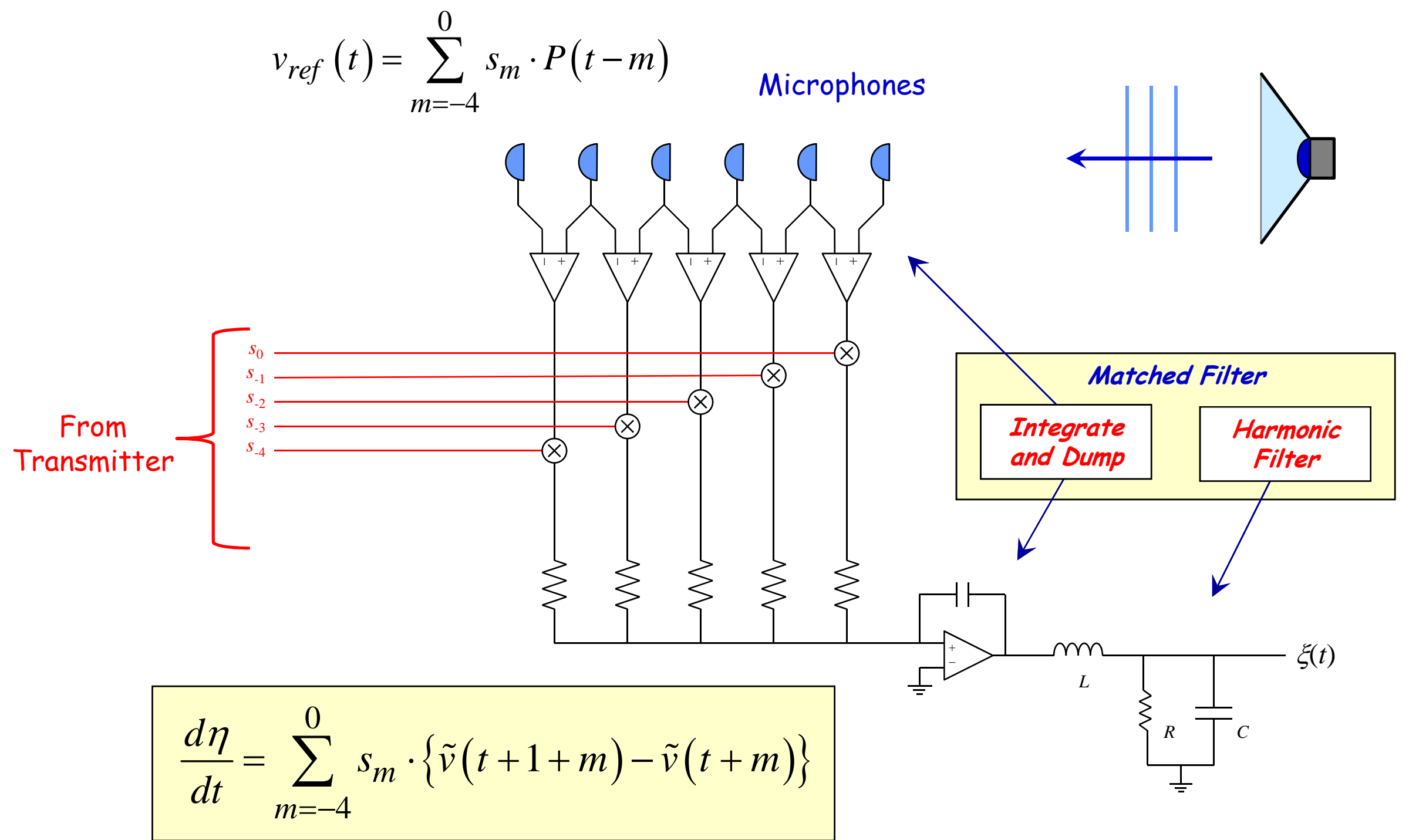




\section{Correlation Receiver}

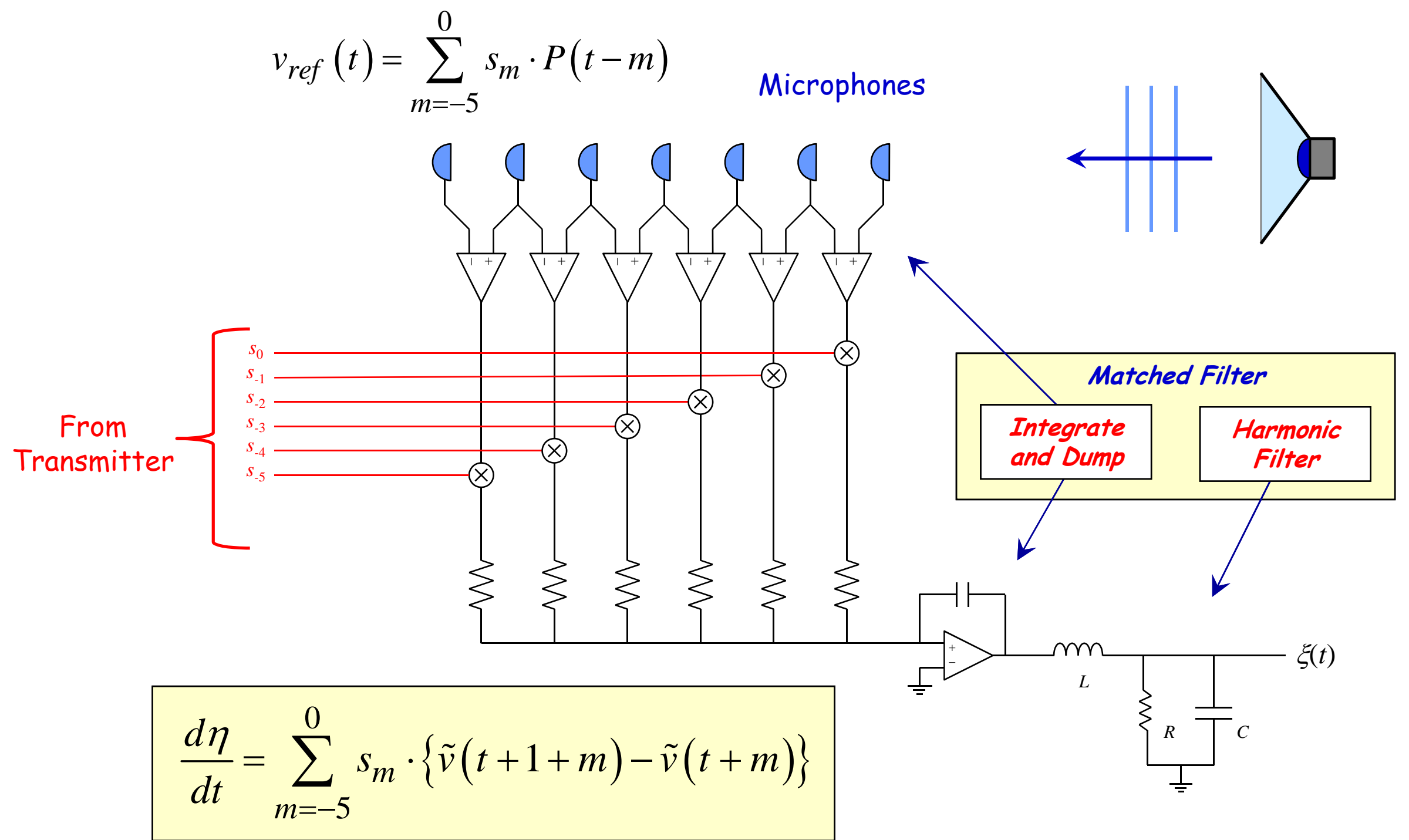




\section{Correlation Receiver}

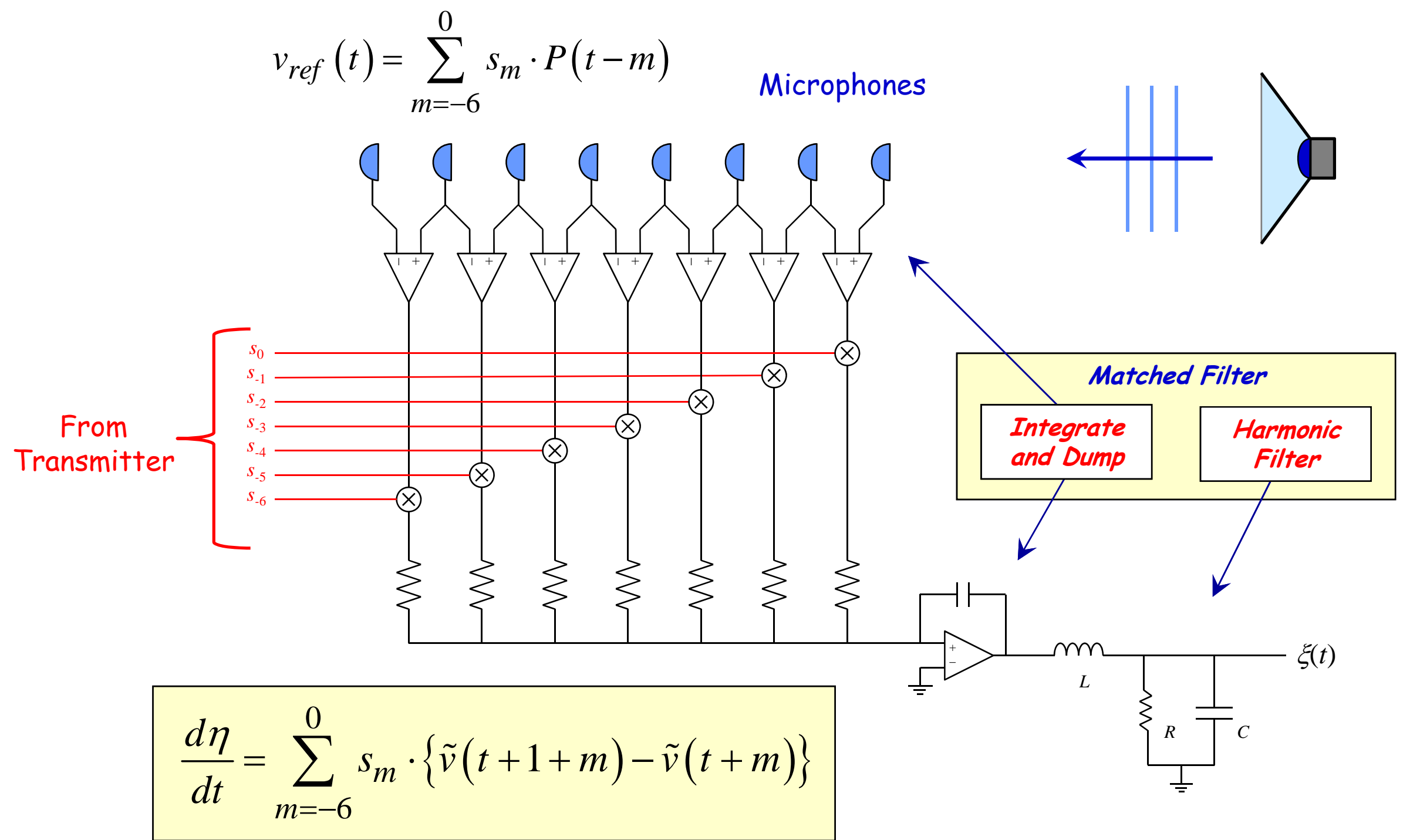




\section{Correlation Receiver}

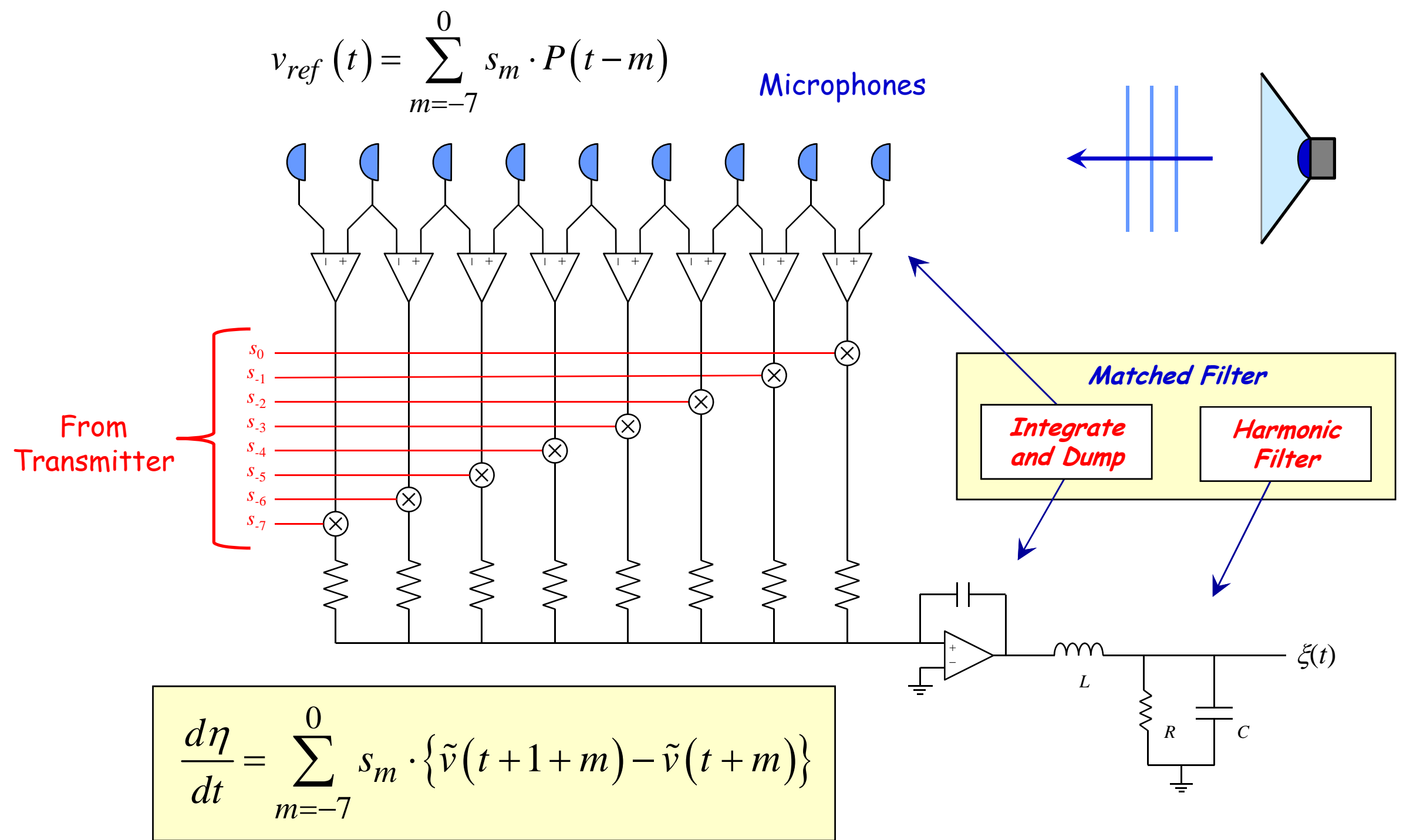




\section{Ranging System}

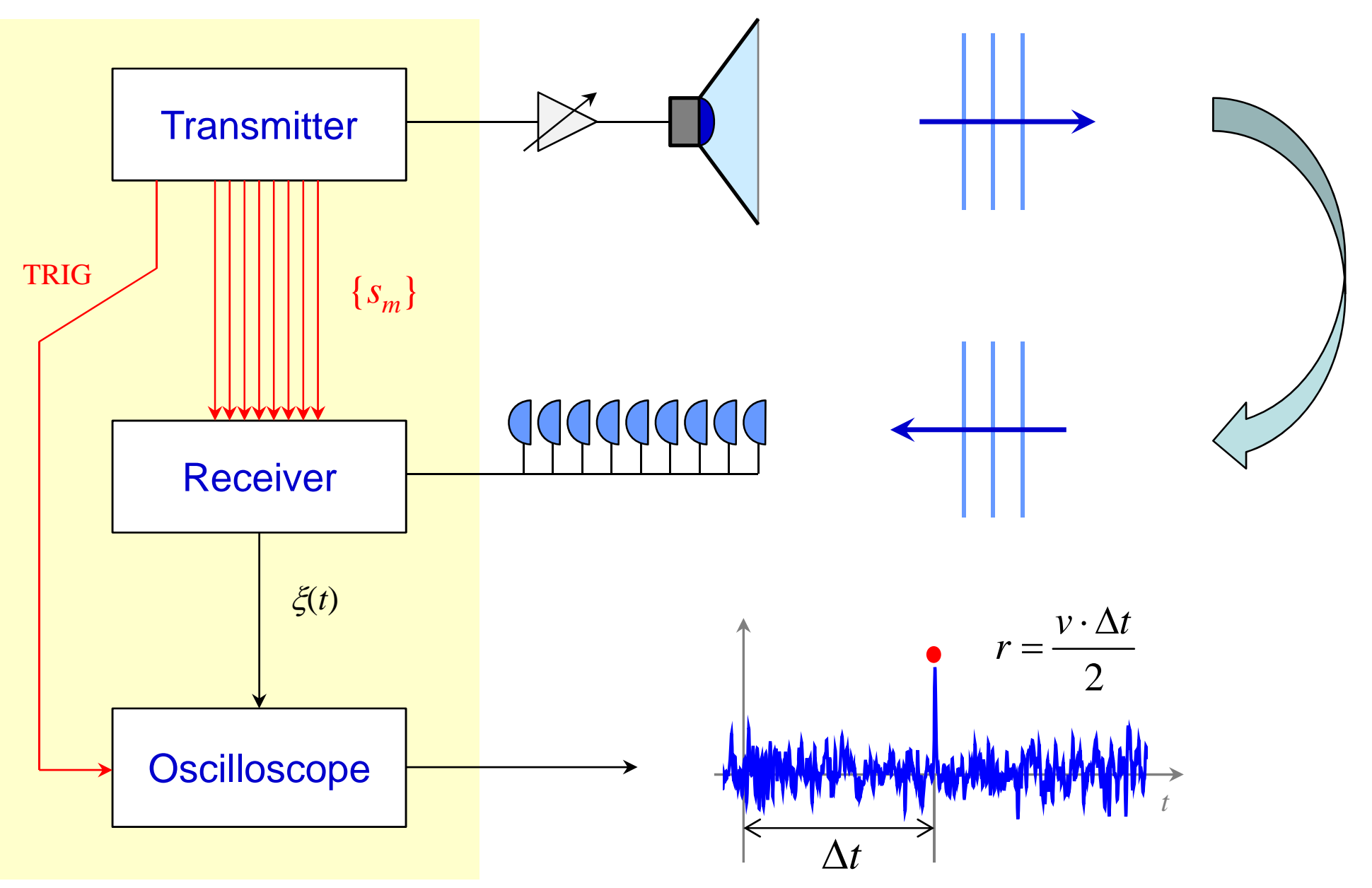




\section{Simulation Waveform 1}
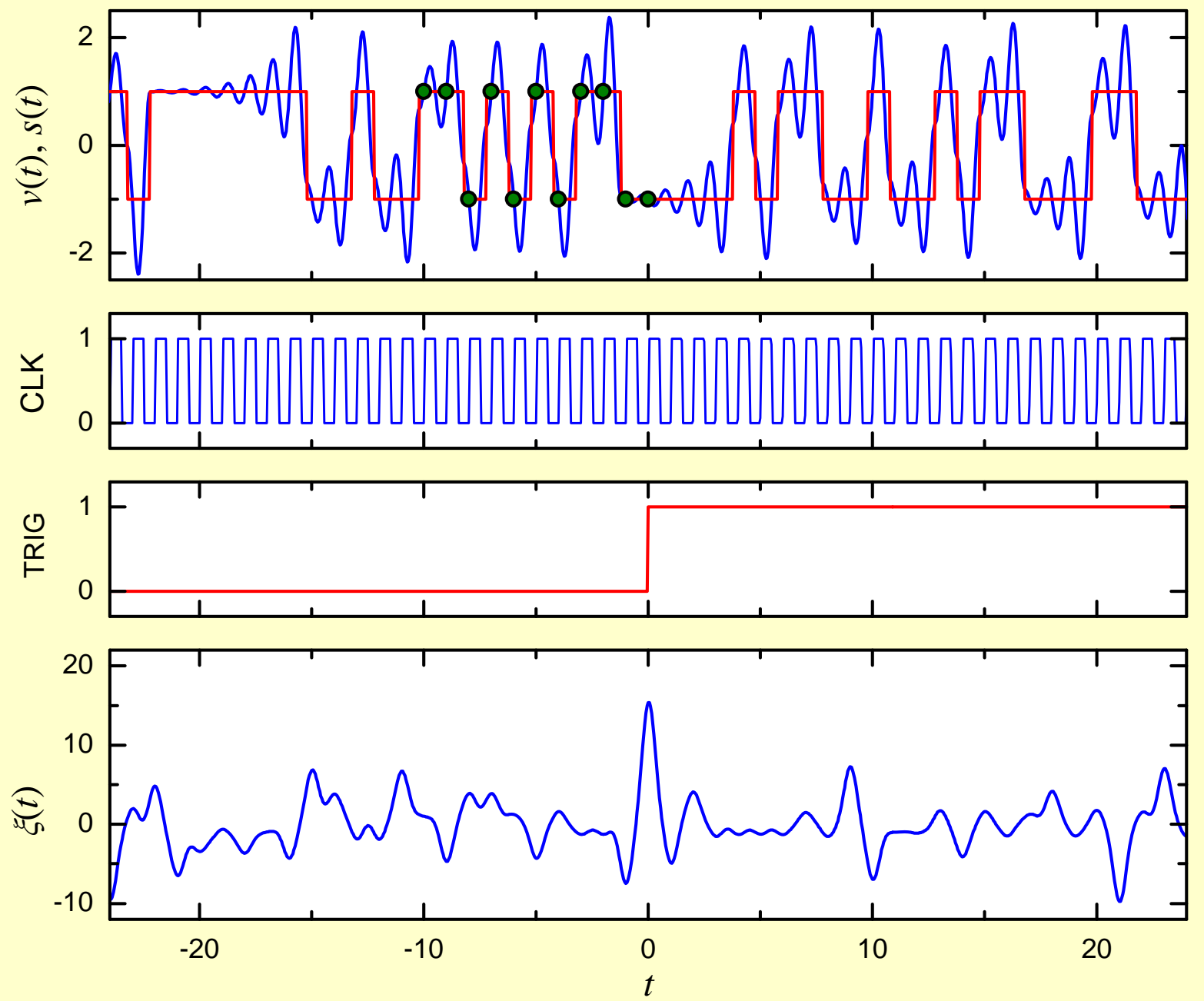


\section{Simulation Waveform 2}
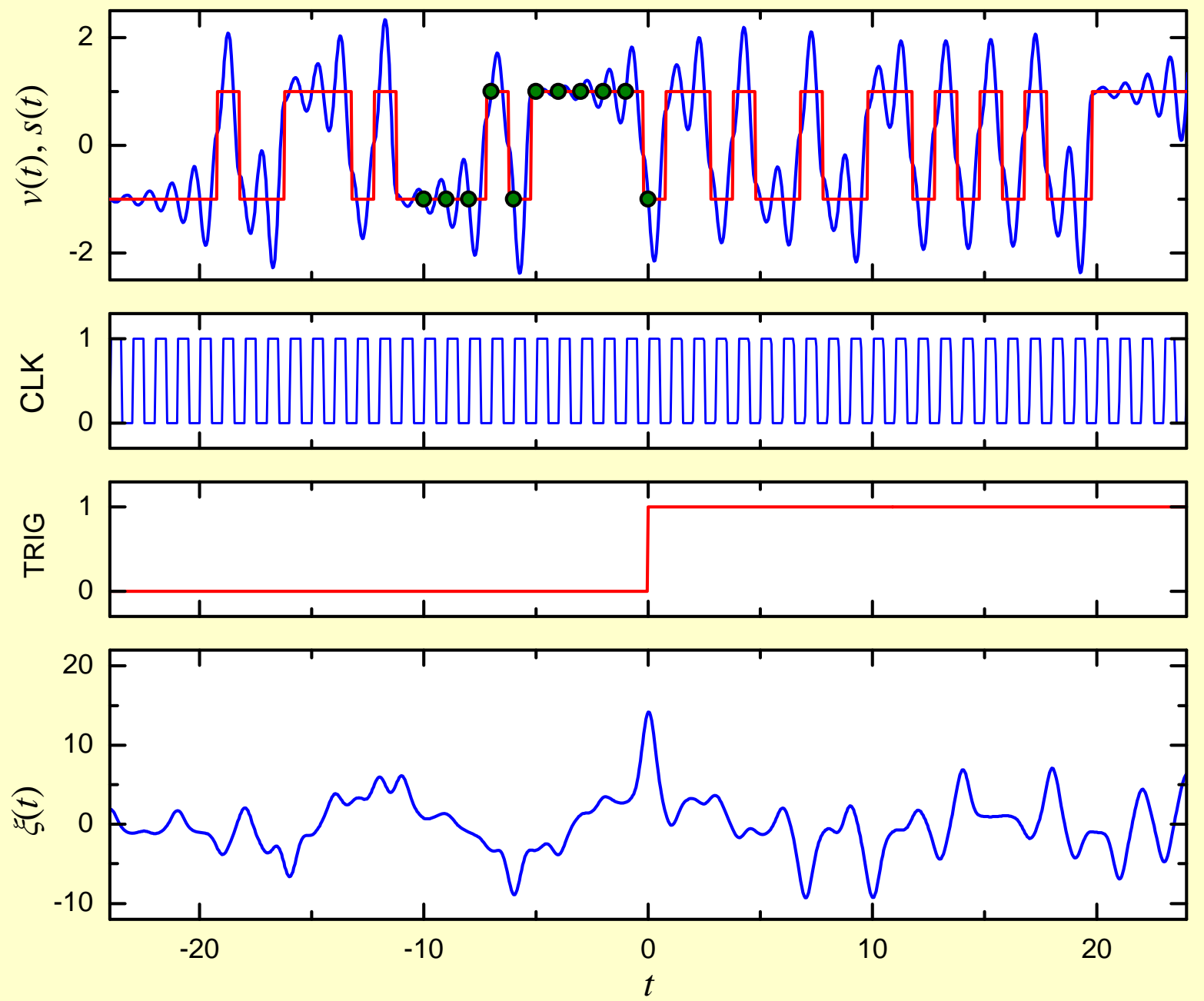


\section{Simulation Waveform 3}
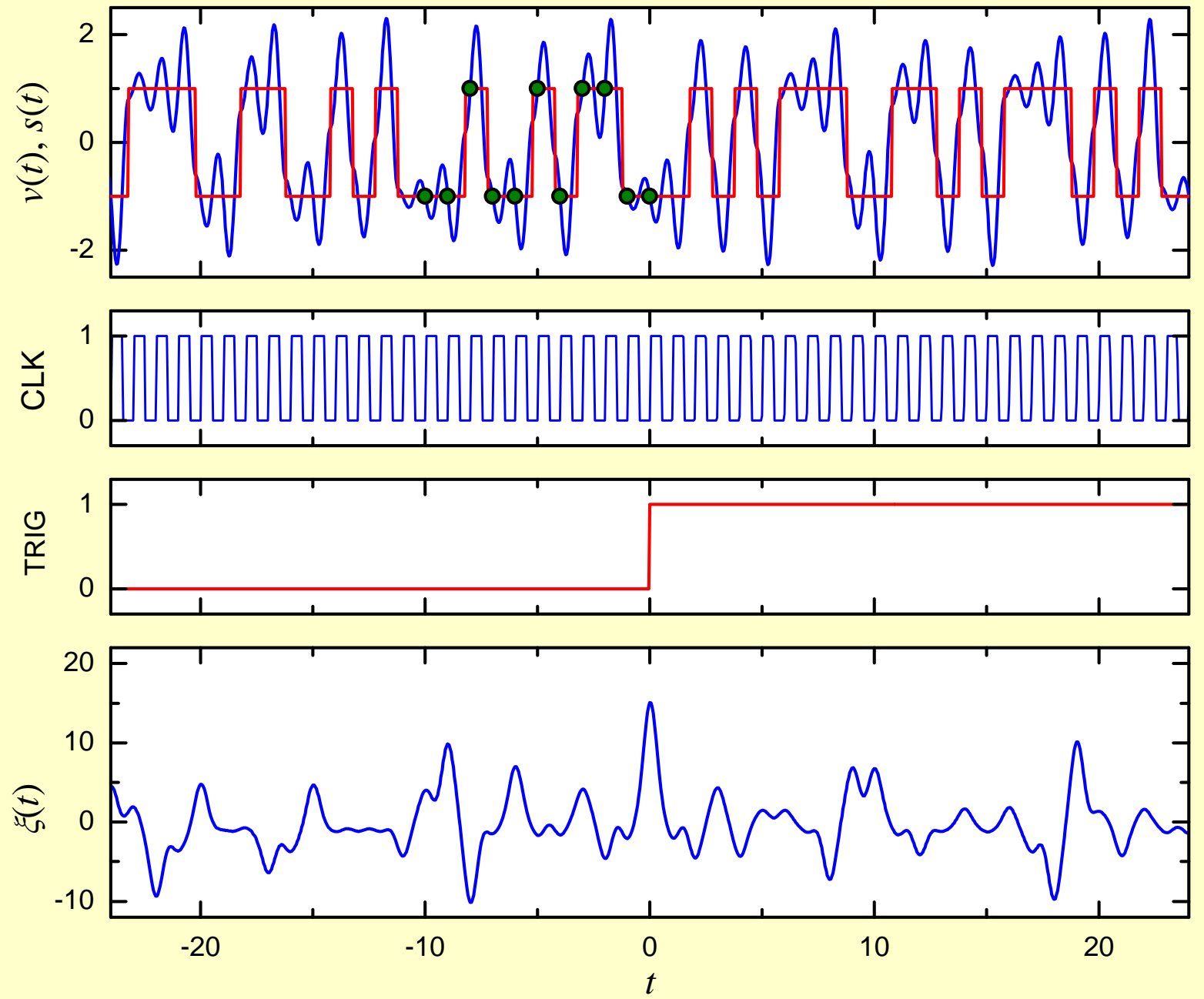


\section{Simulation Waveform 4}
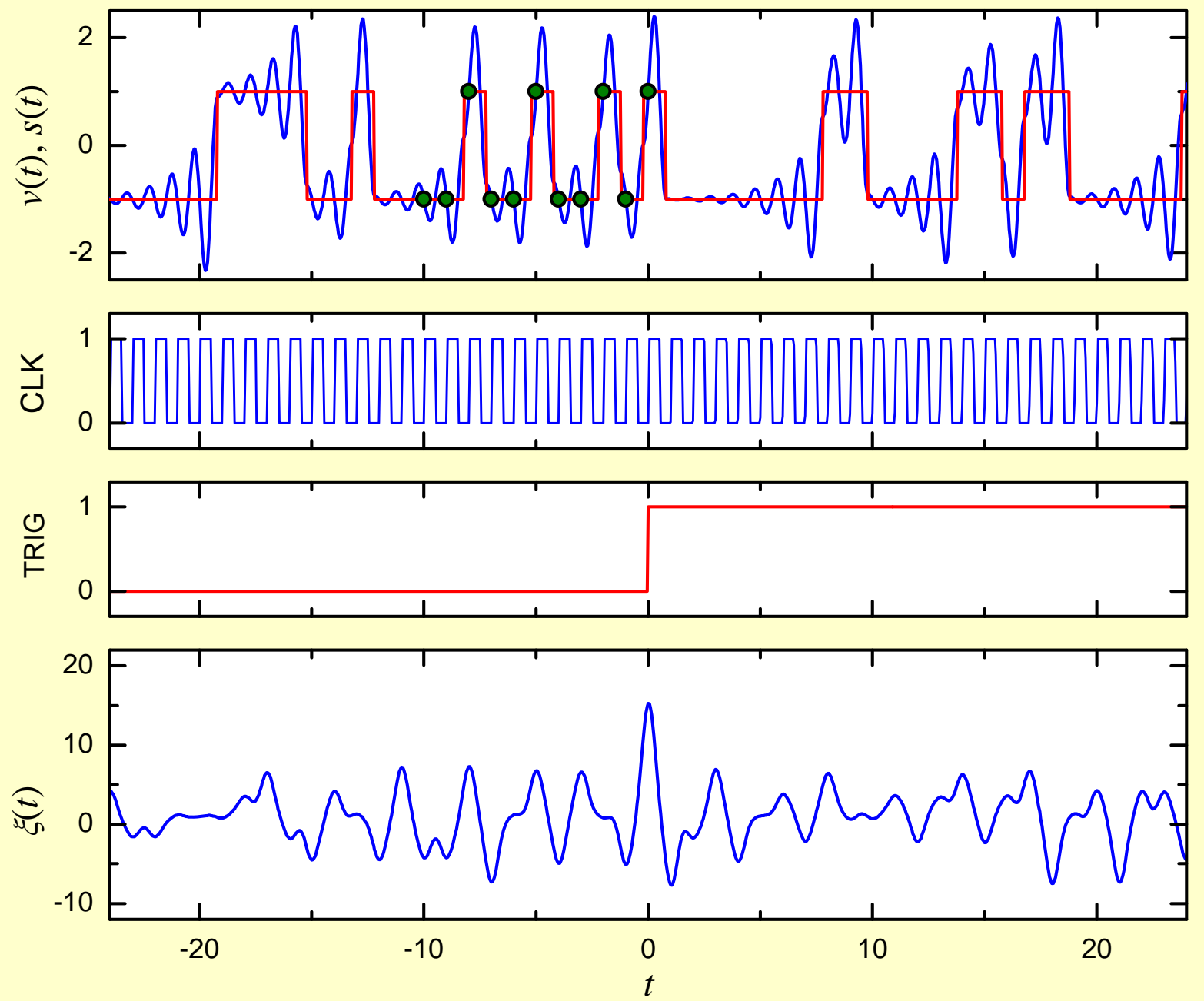


\section{Simulation Waveform 5}
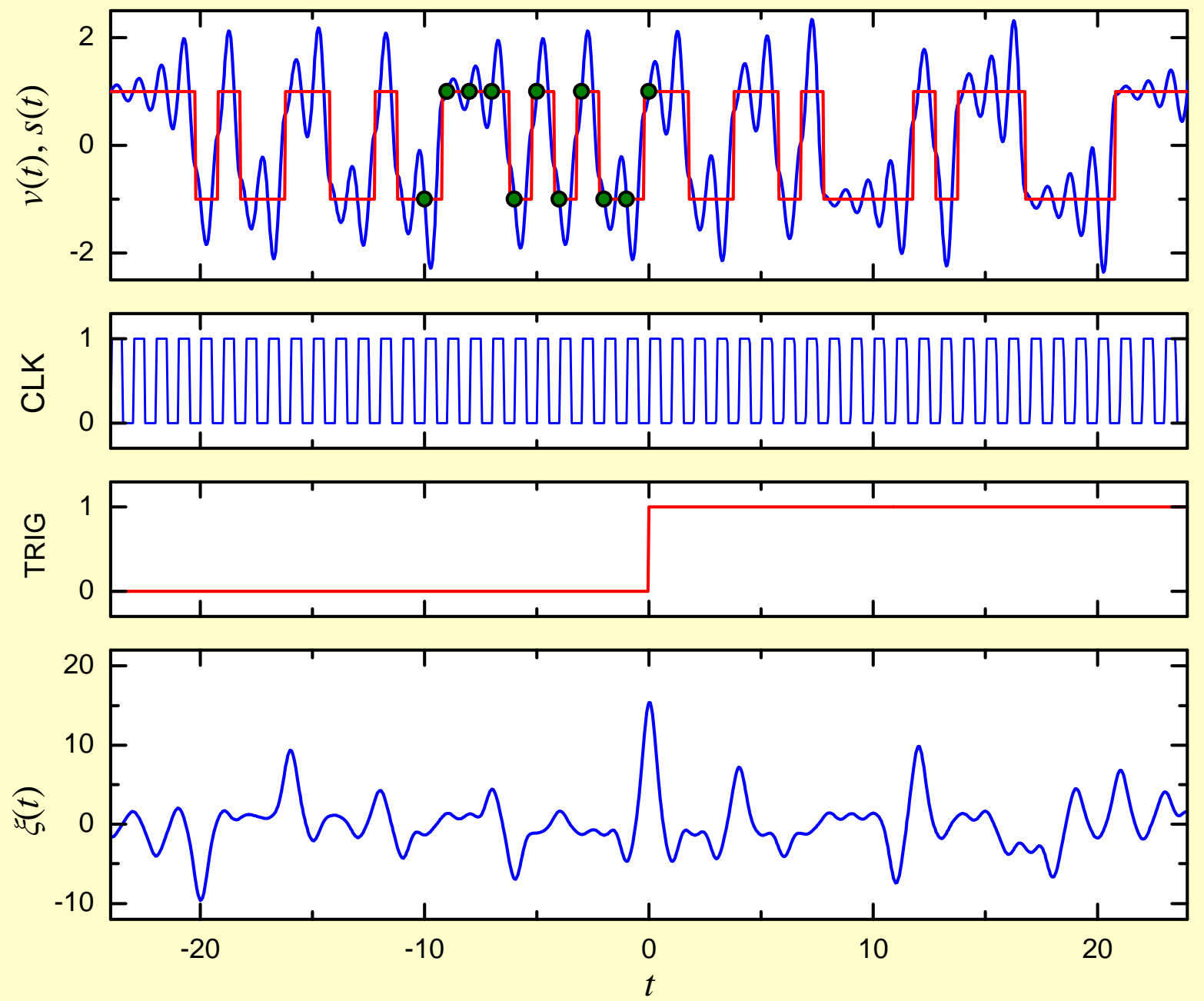


\section{Simulation Waveform 6}
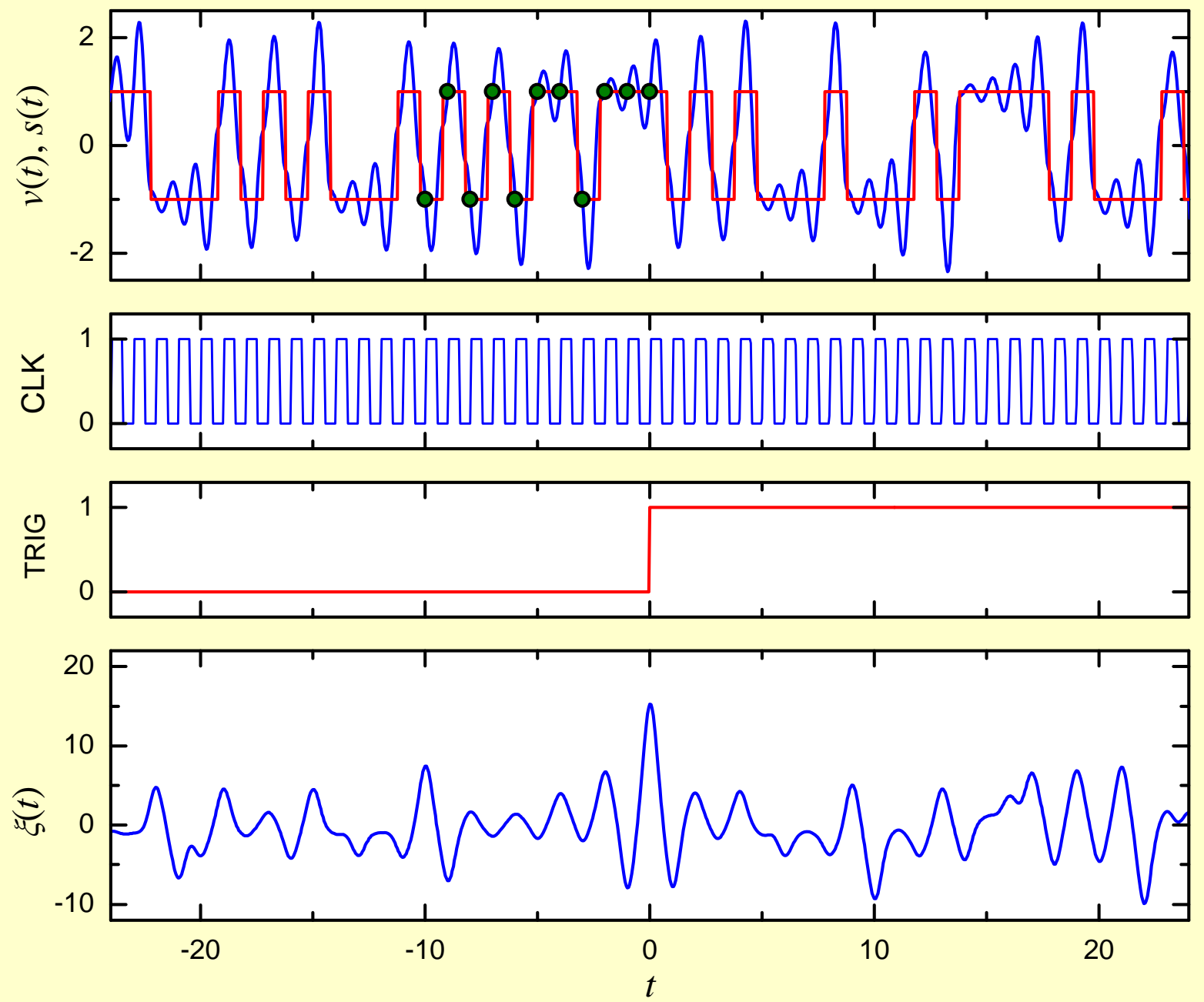


\section{Simulation Waveform 7}
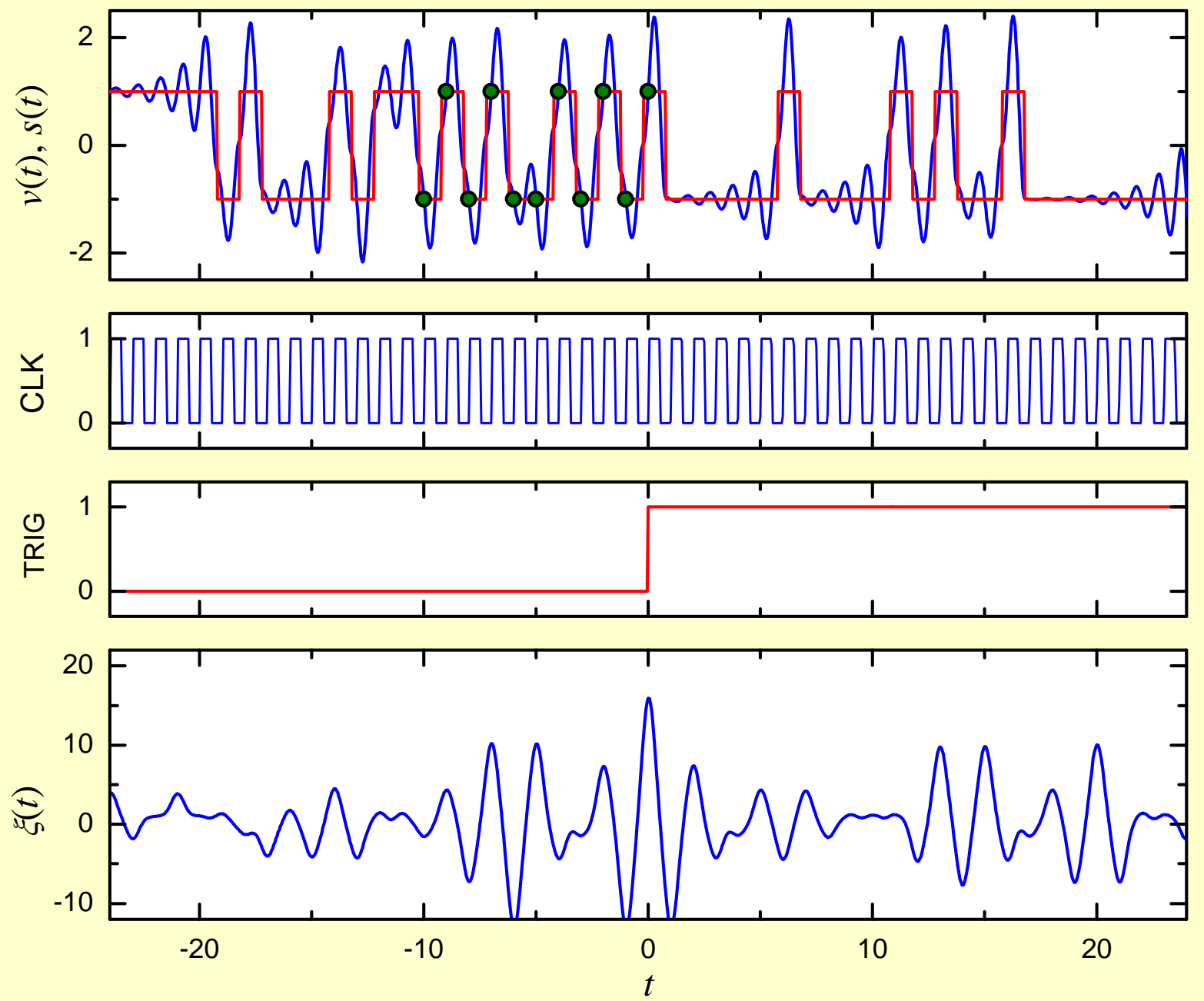


\section{Simulation Waveform 8}
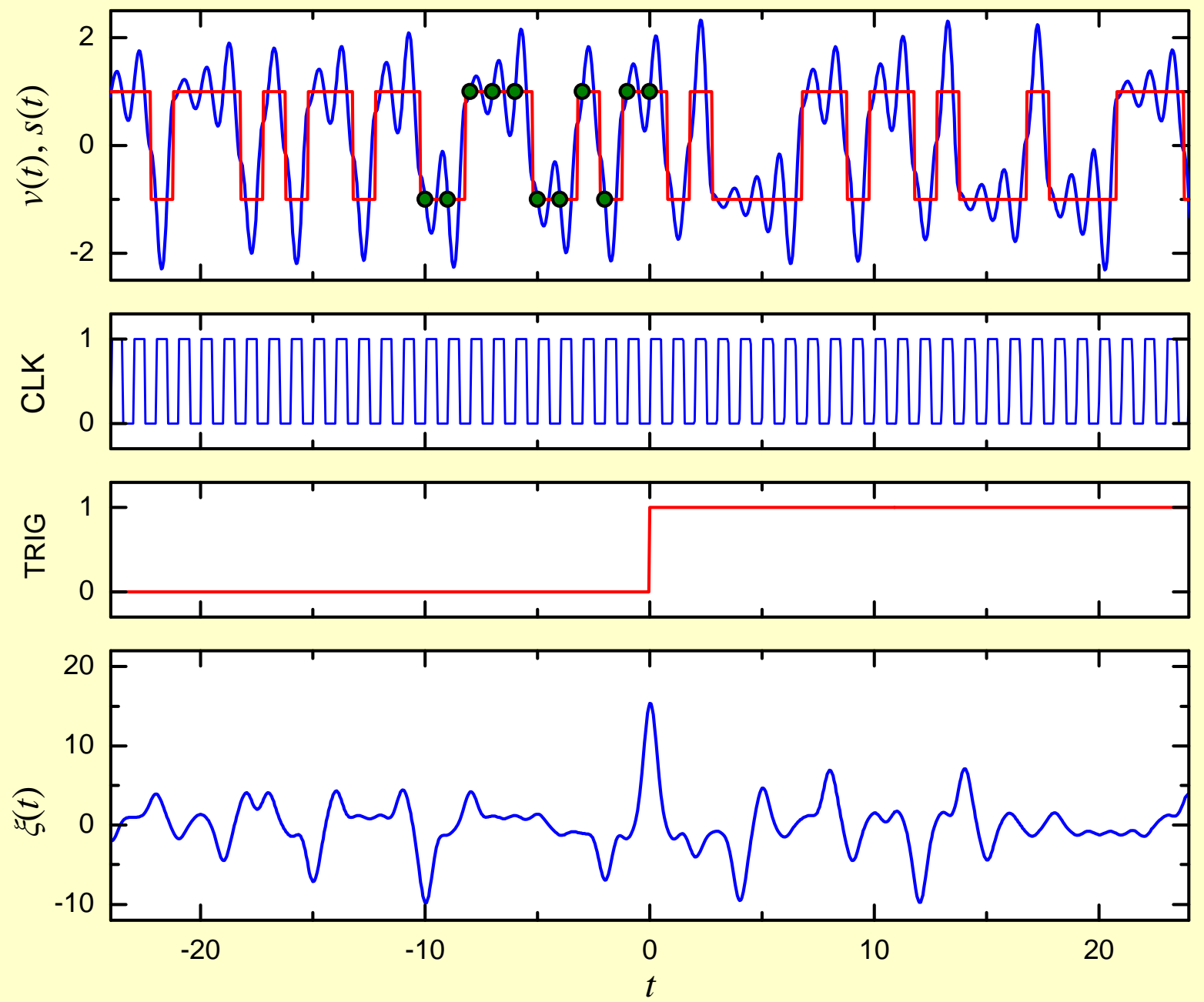


\section{Simulation Waveform 9}
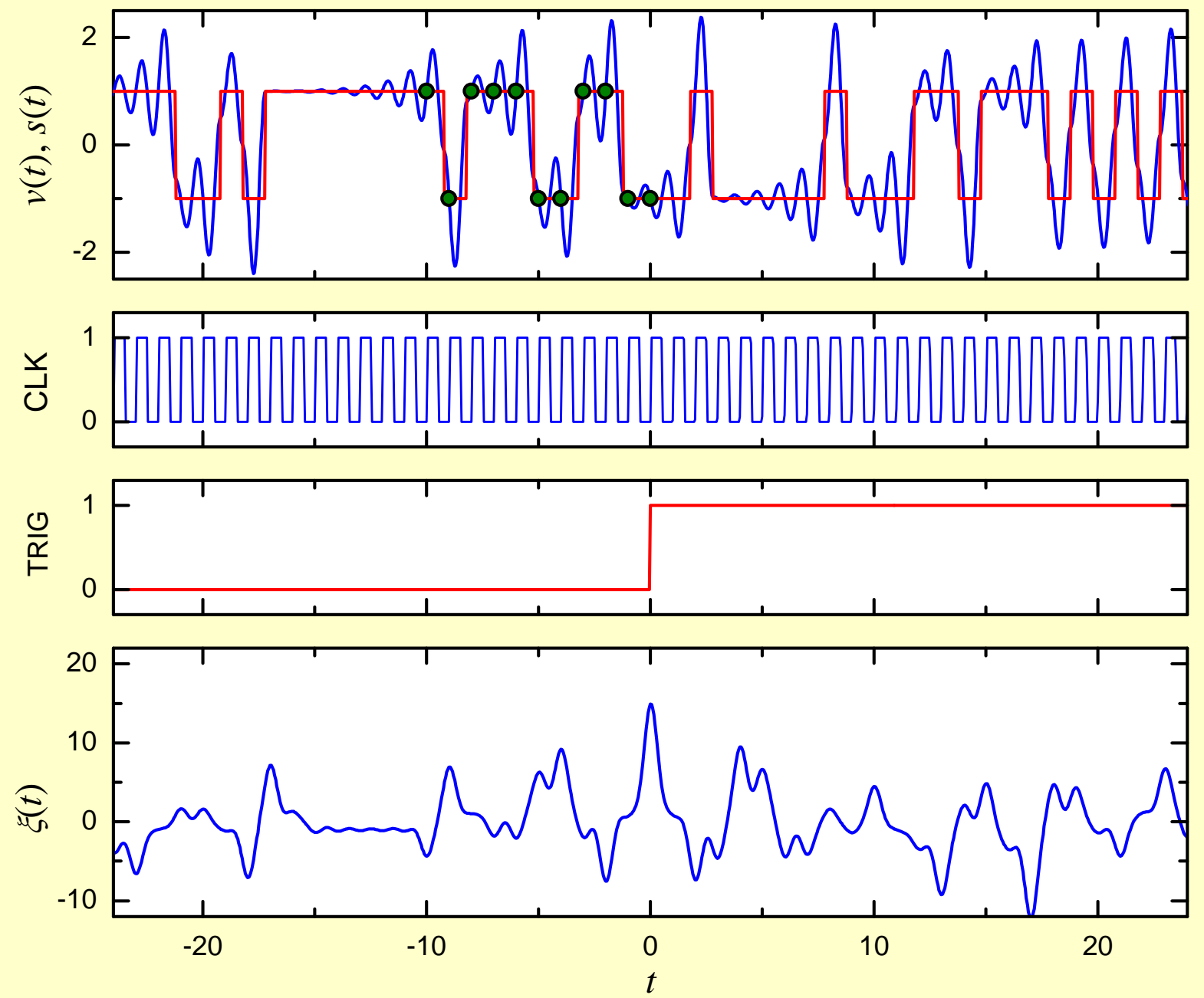


\section{Simulation Averaged Matched Filter}
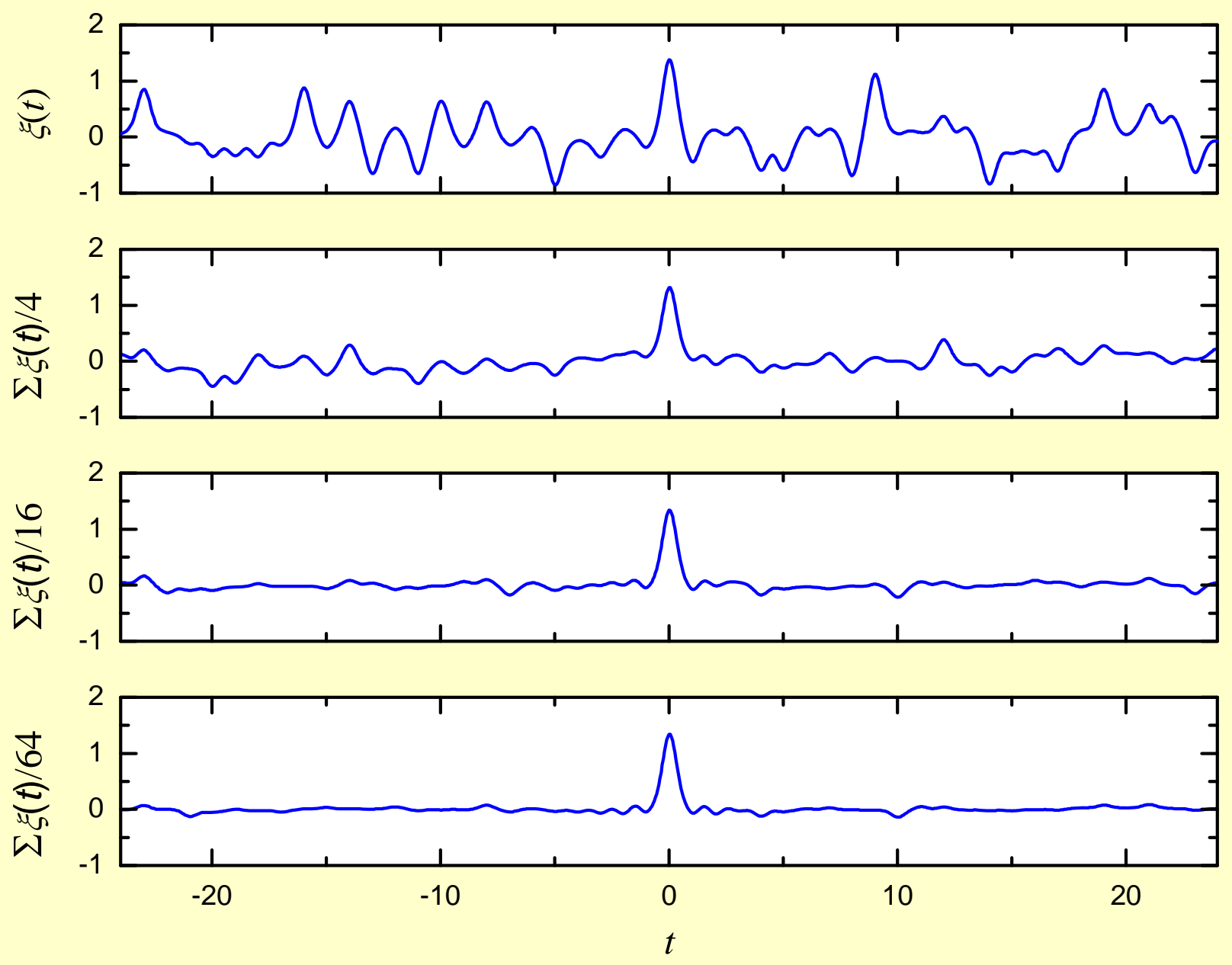


\section{Experimental System}
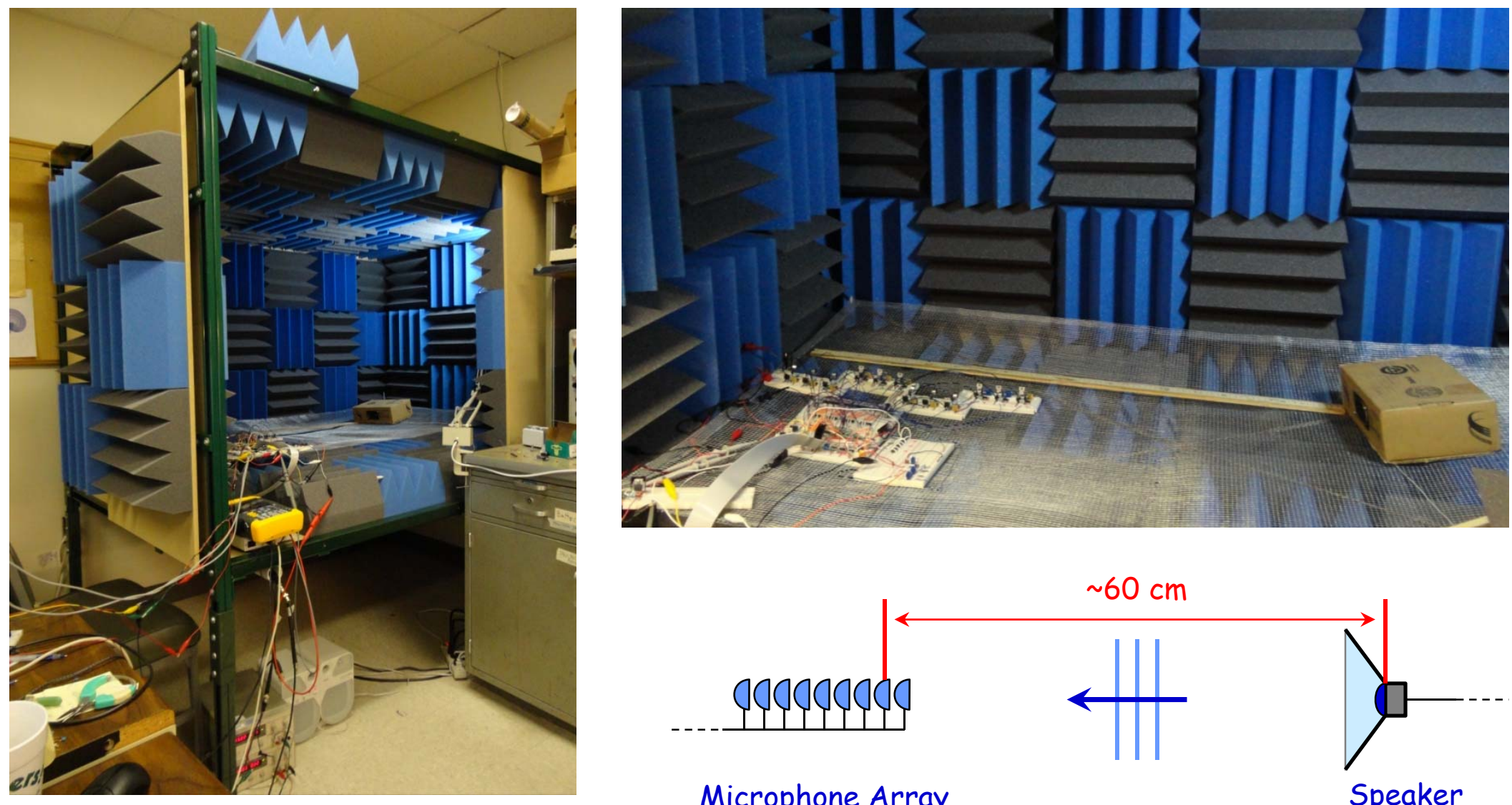

Microphone Array

Speaker 


\section{Experimental System}

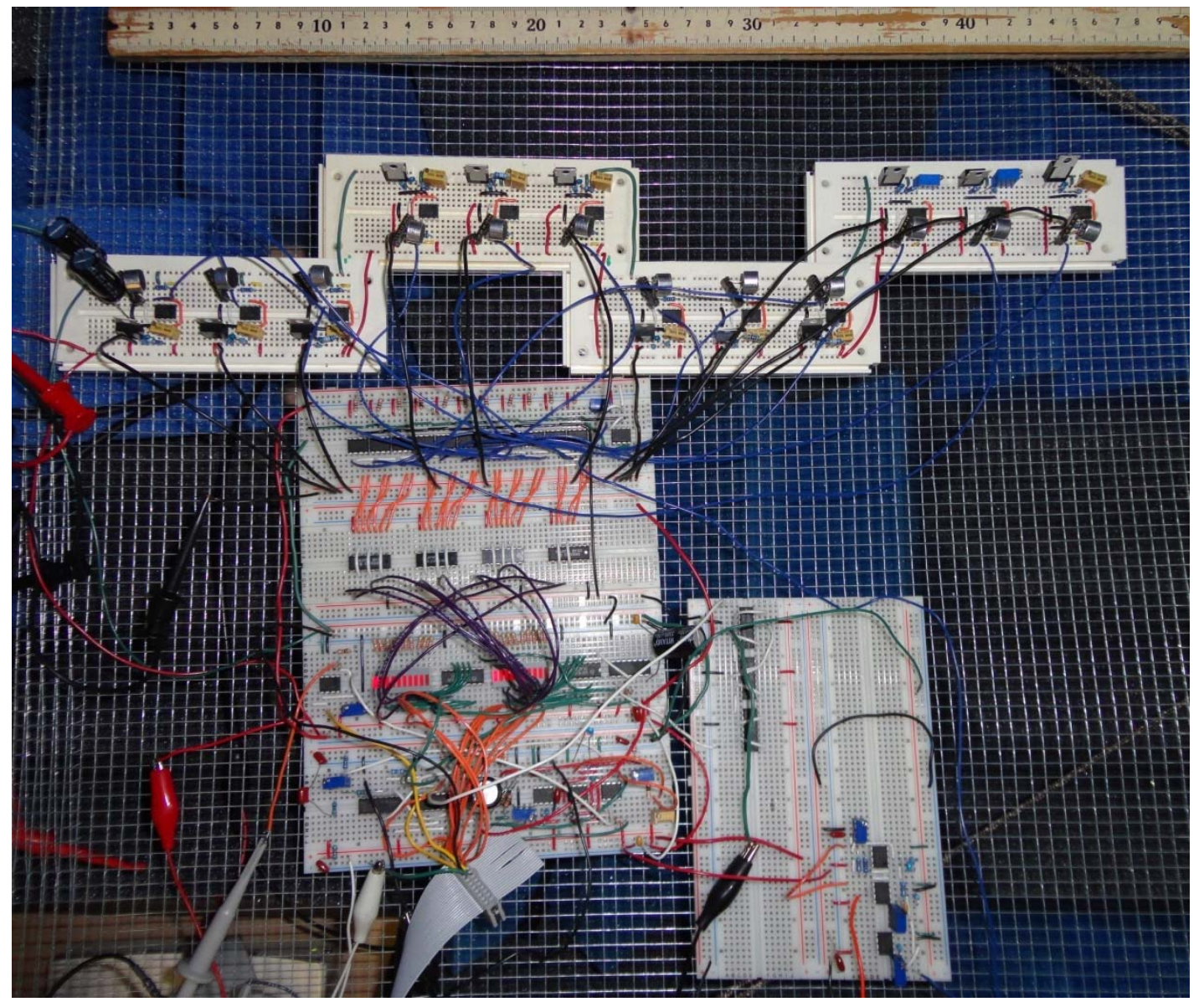

oscillator clock $\sim 10 \mathrm{kHz} \rightarrow 3-\mathrm{cm}$ microphone spacing
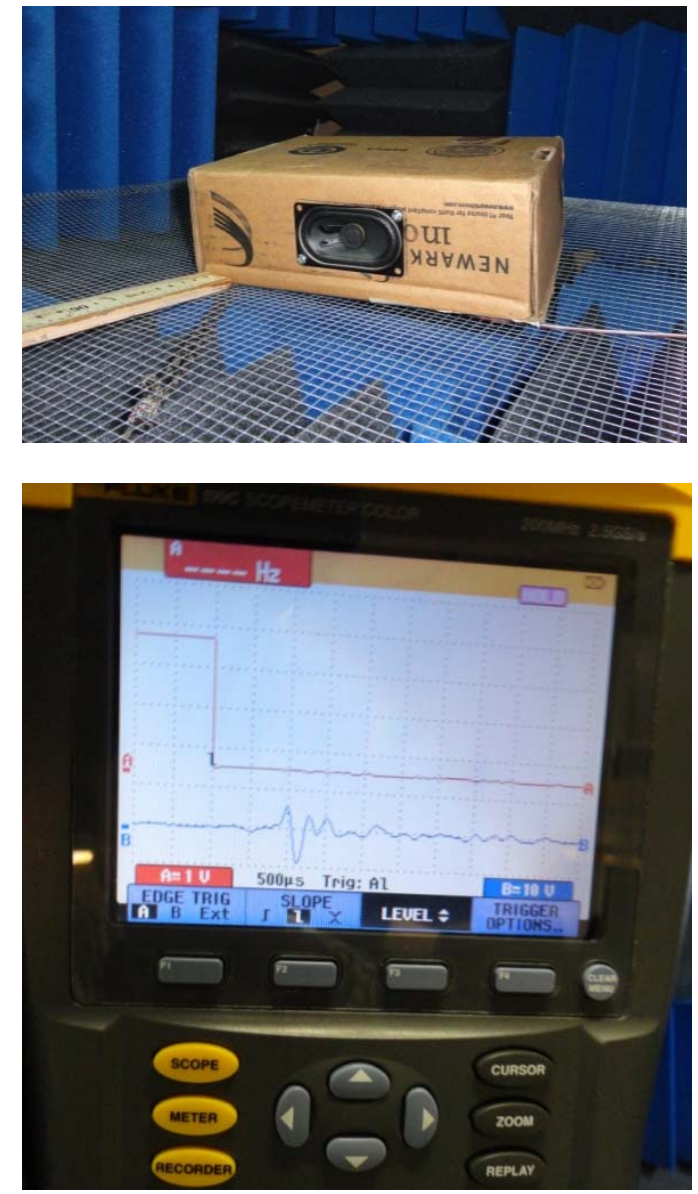

oscilloscope averaging 


\section{Measured Waveform 1}

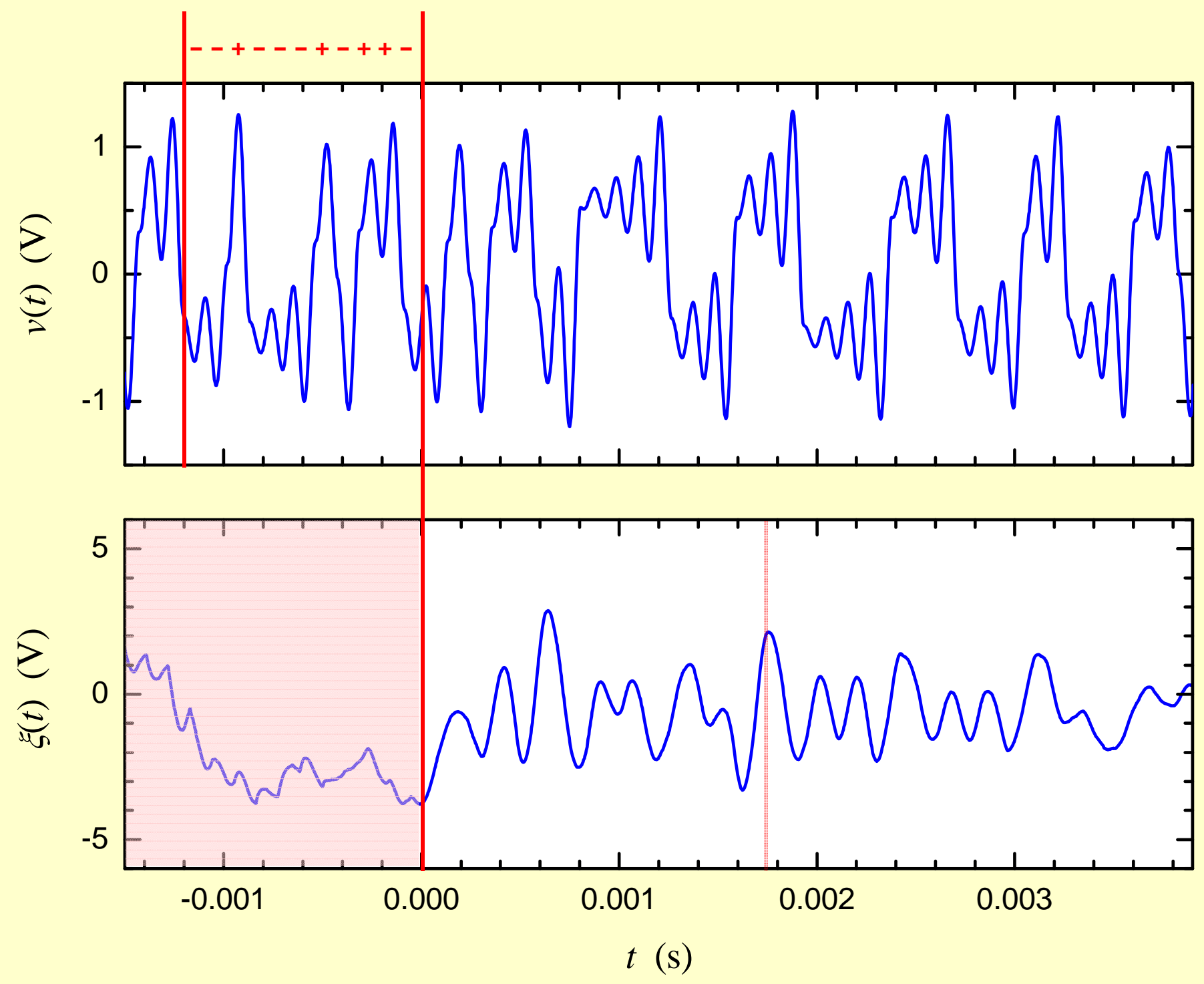




\section{Measured Waveform 2}

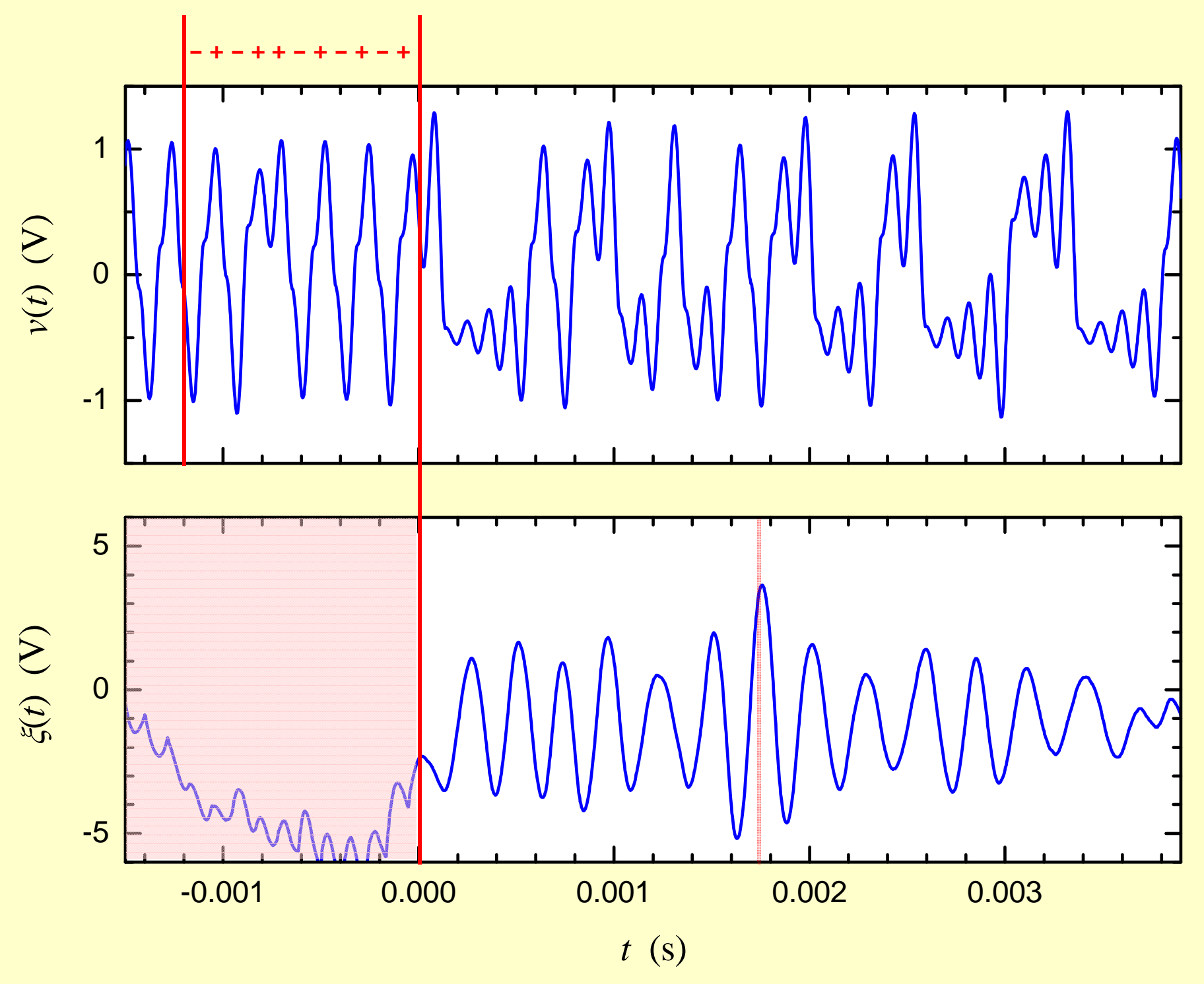




\section{Measured Waveform 3}

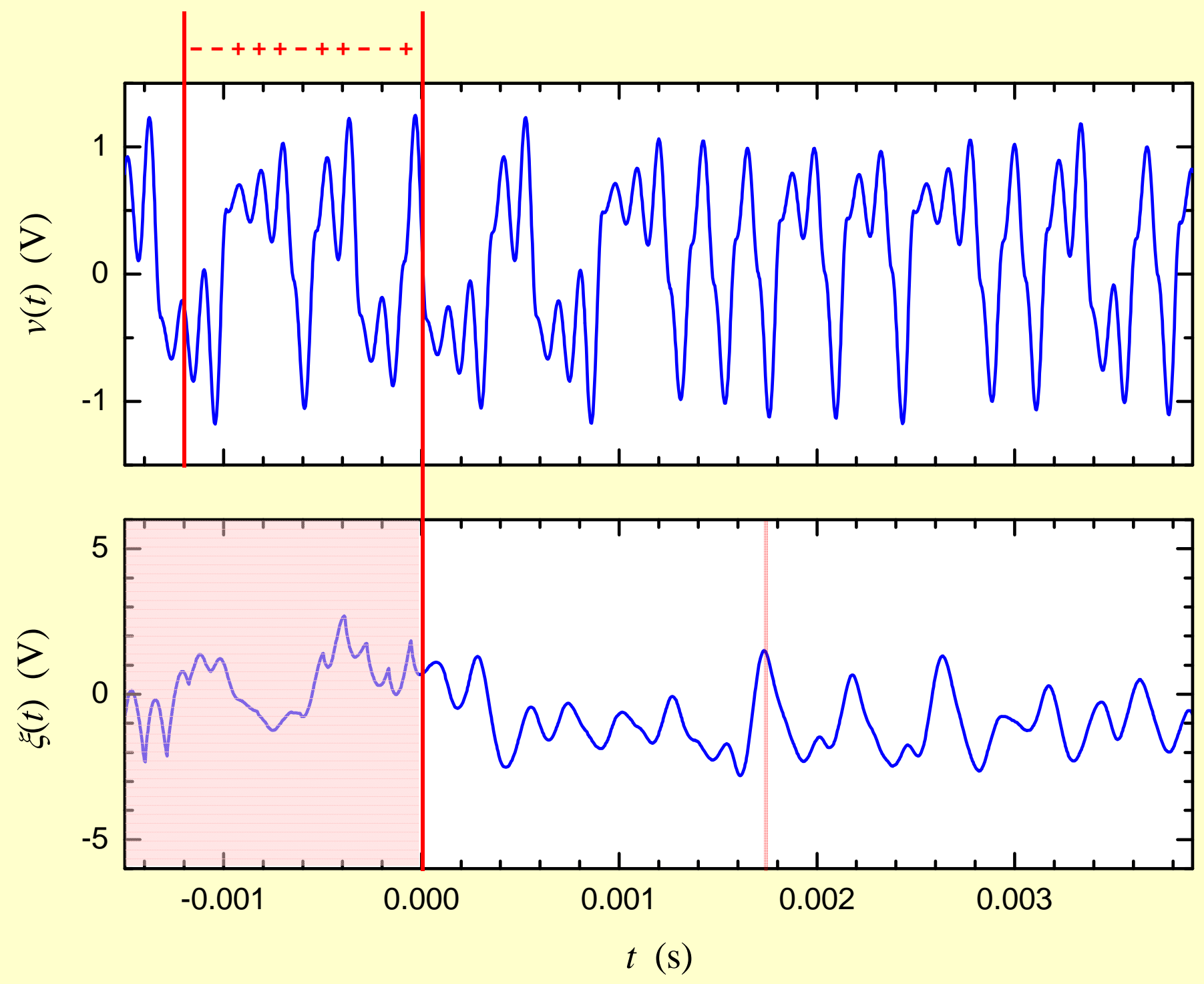




\section{Measured Waveform 4}

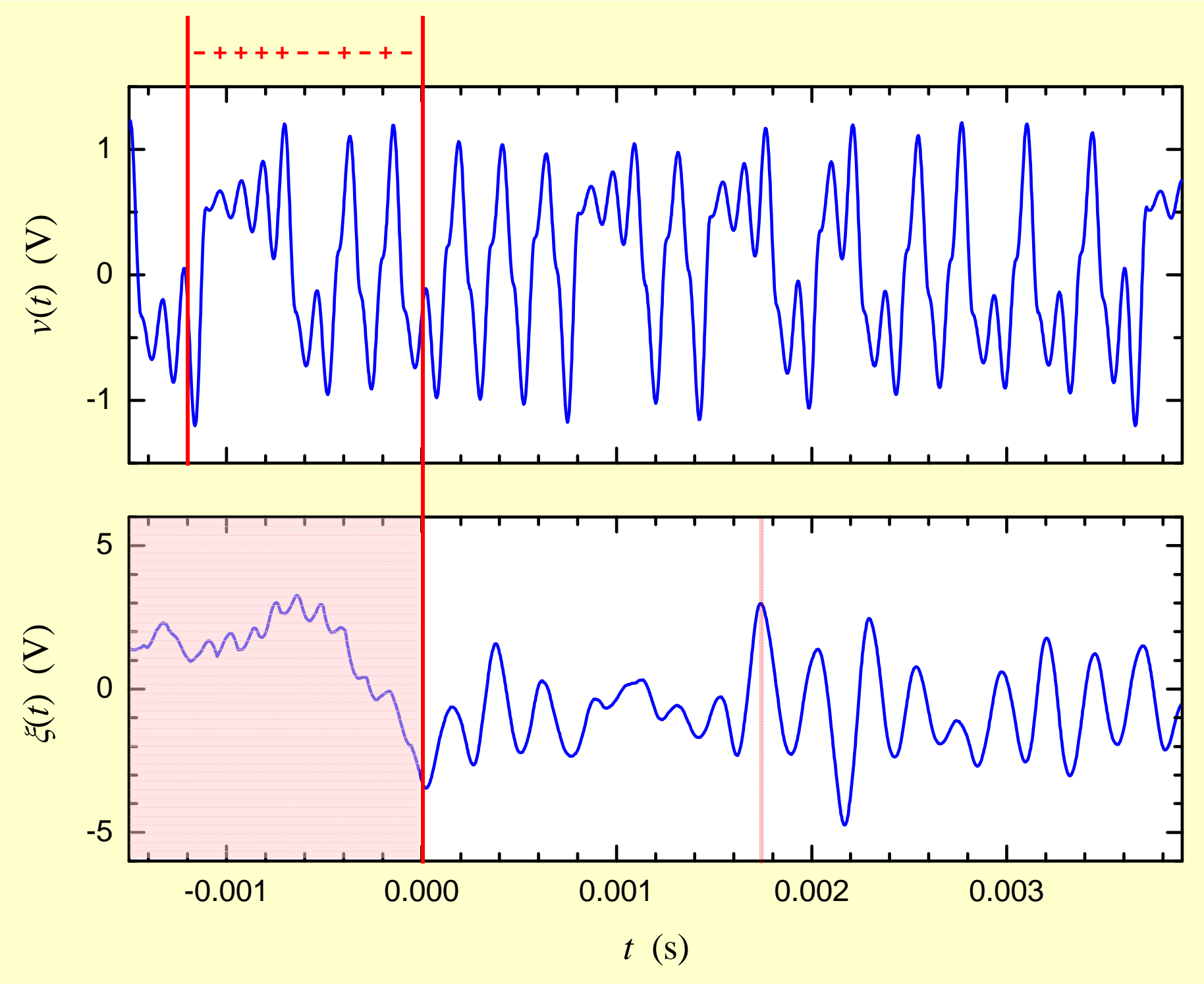




\section{Measured Waveform 5}

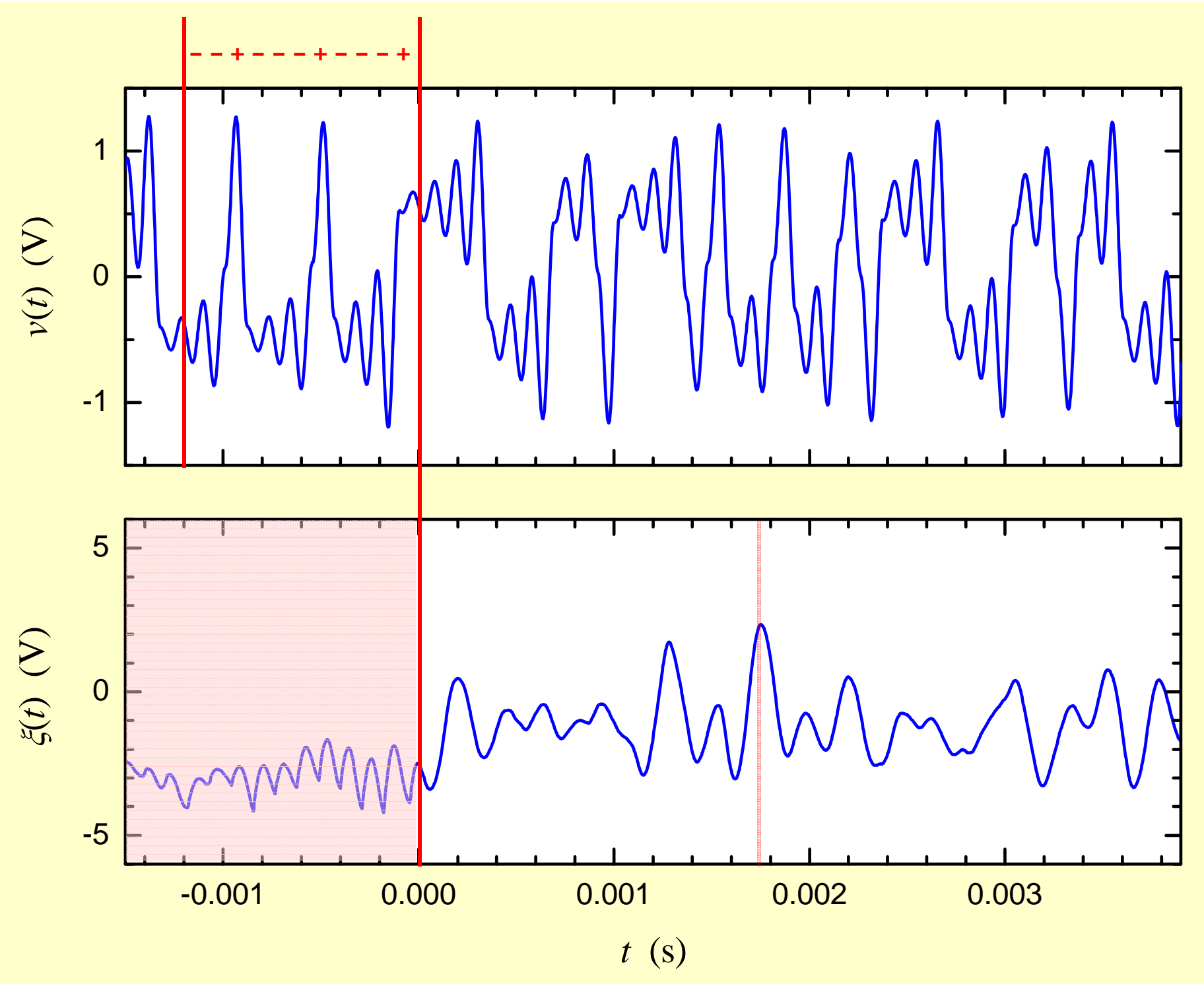




\section{Measured Waveform 6}

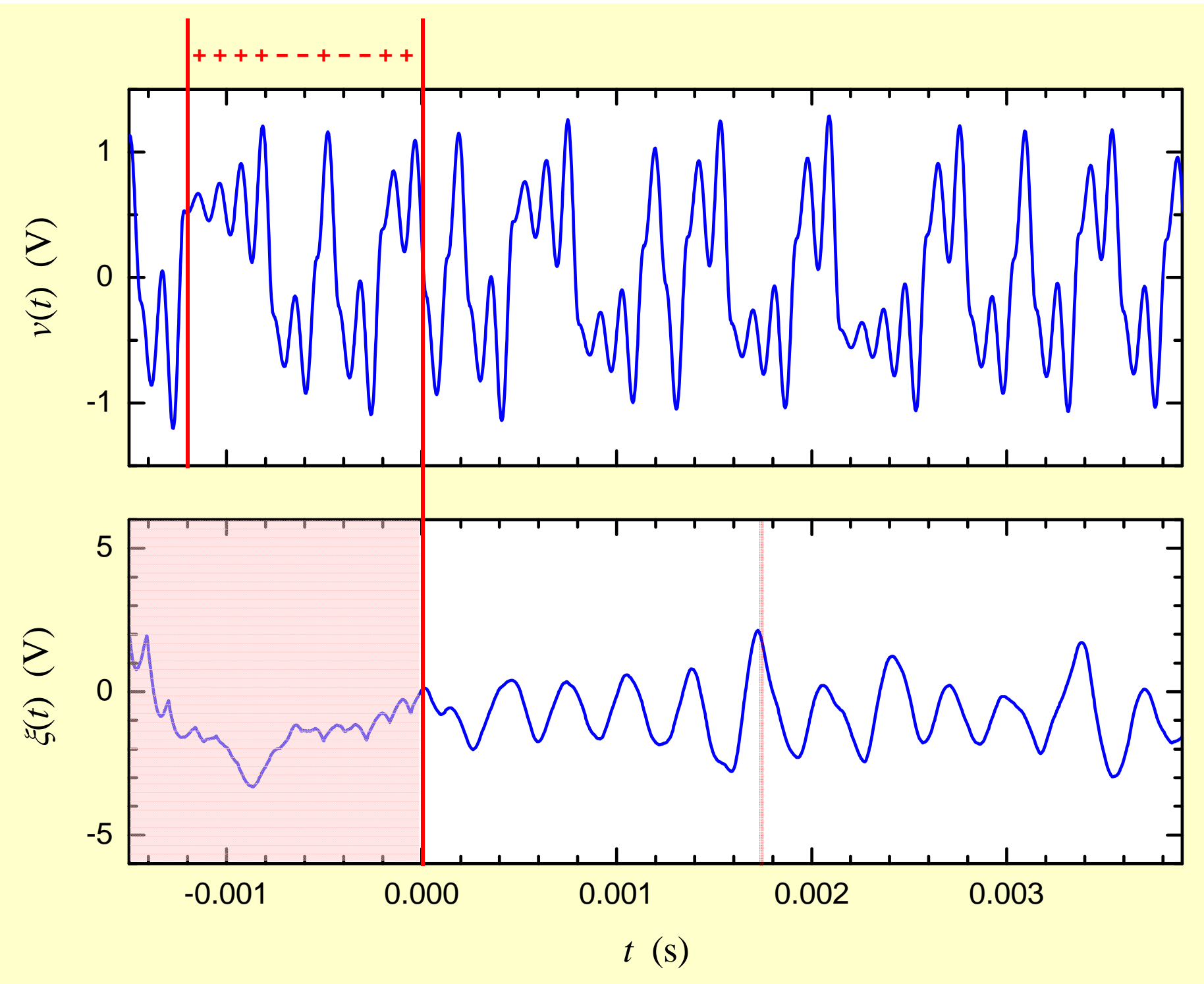




\section{Measured Waveform 7}

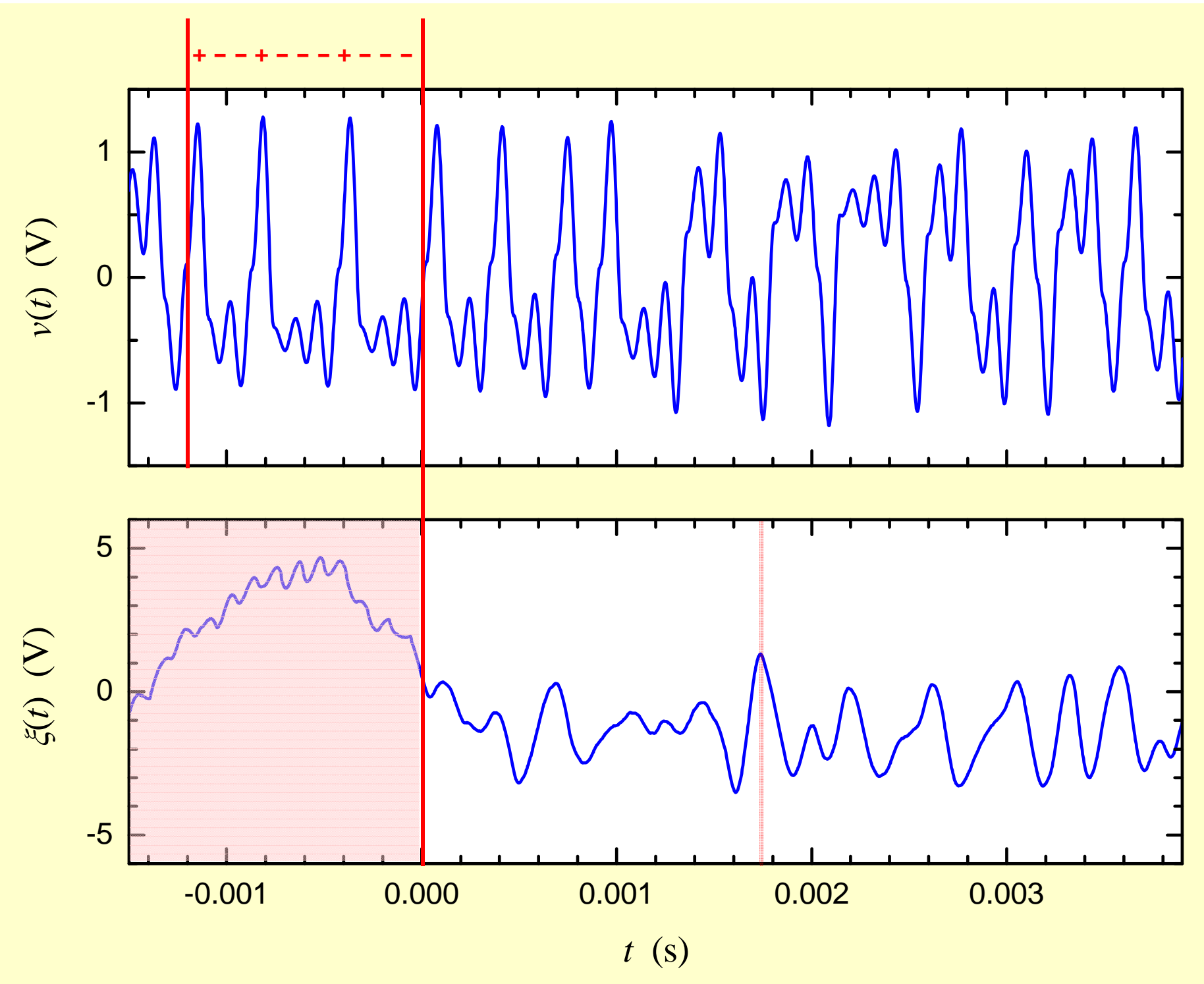




\section{Measured Waveform 8}

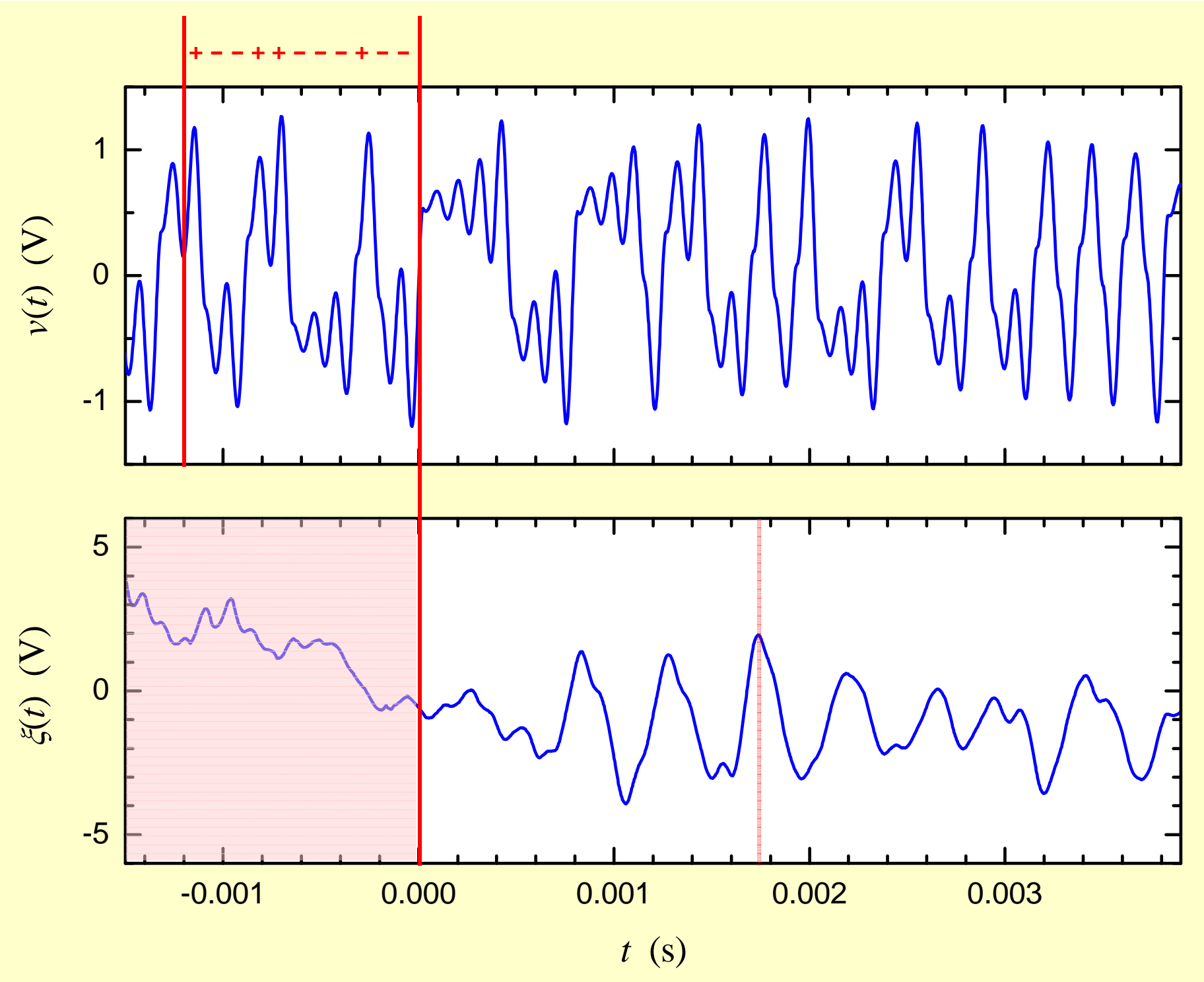




\section{Measured Waveform 9}

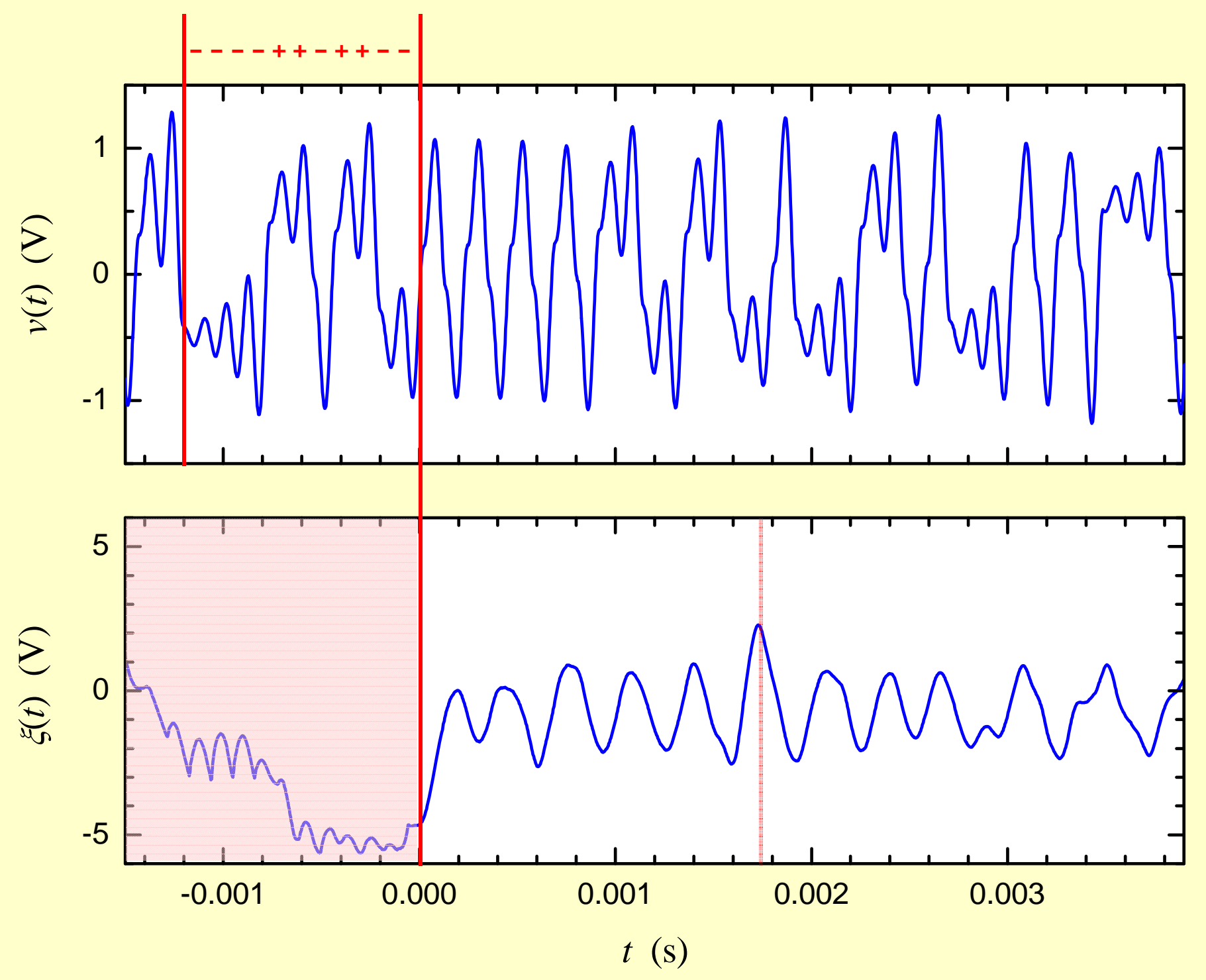




\section{Measured Average Matched Filter}
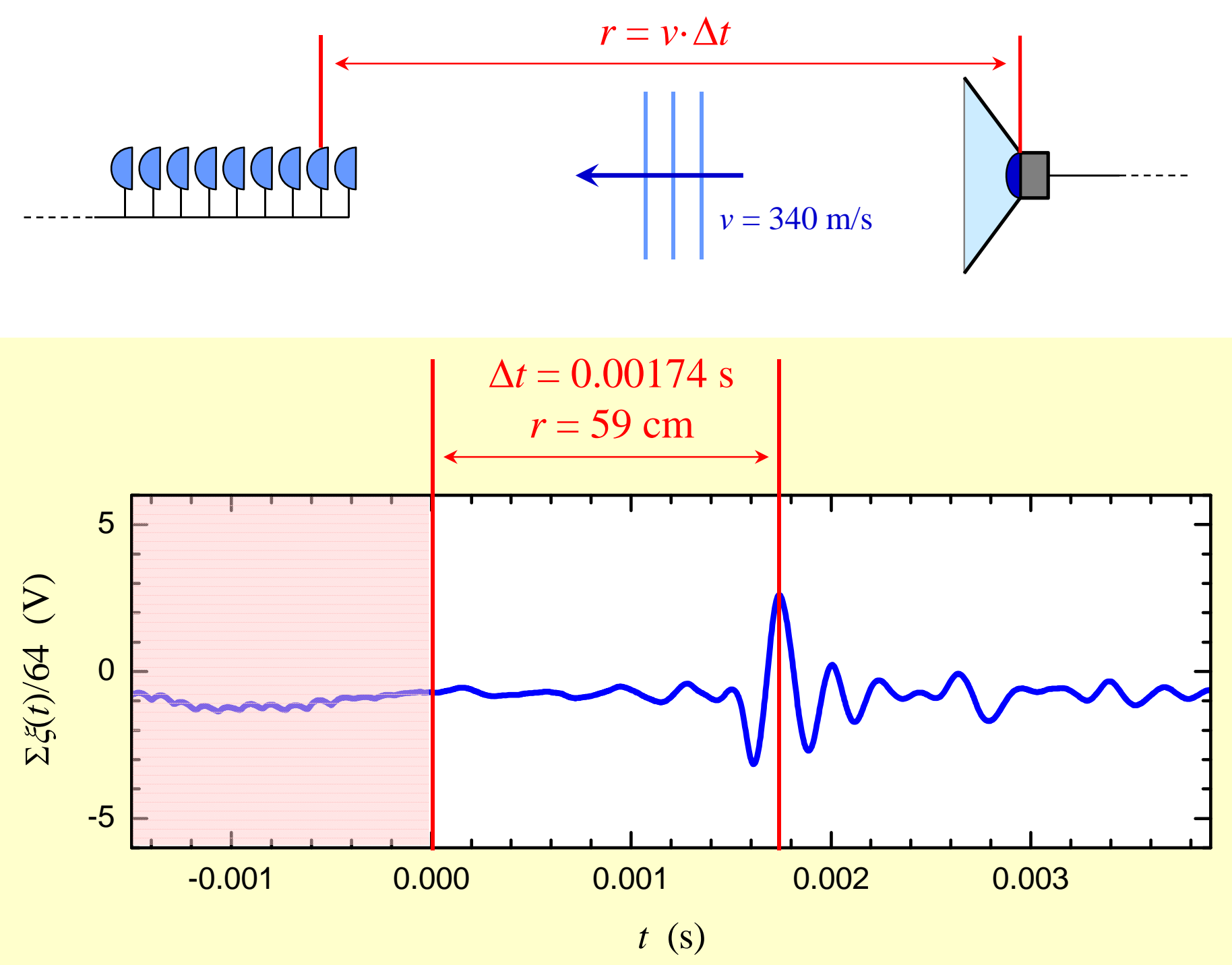


\section{Range Measurements}

Varied Speaker Positions:

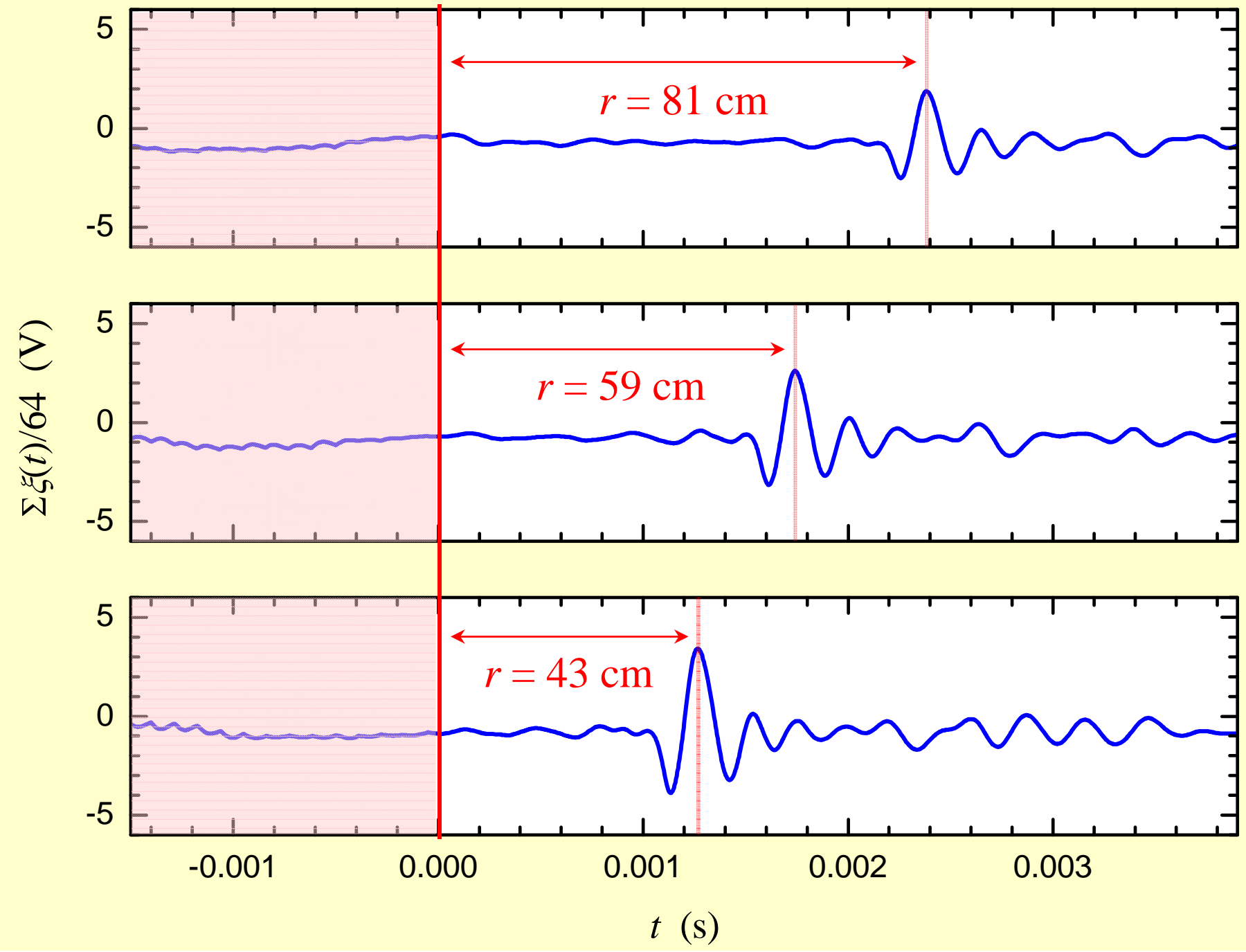




\section{Noise Radar}

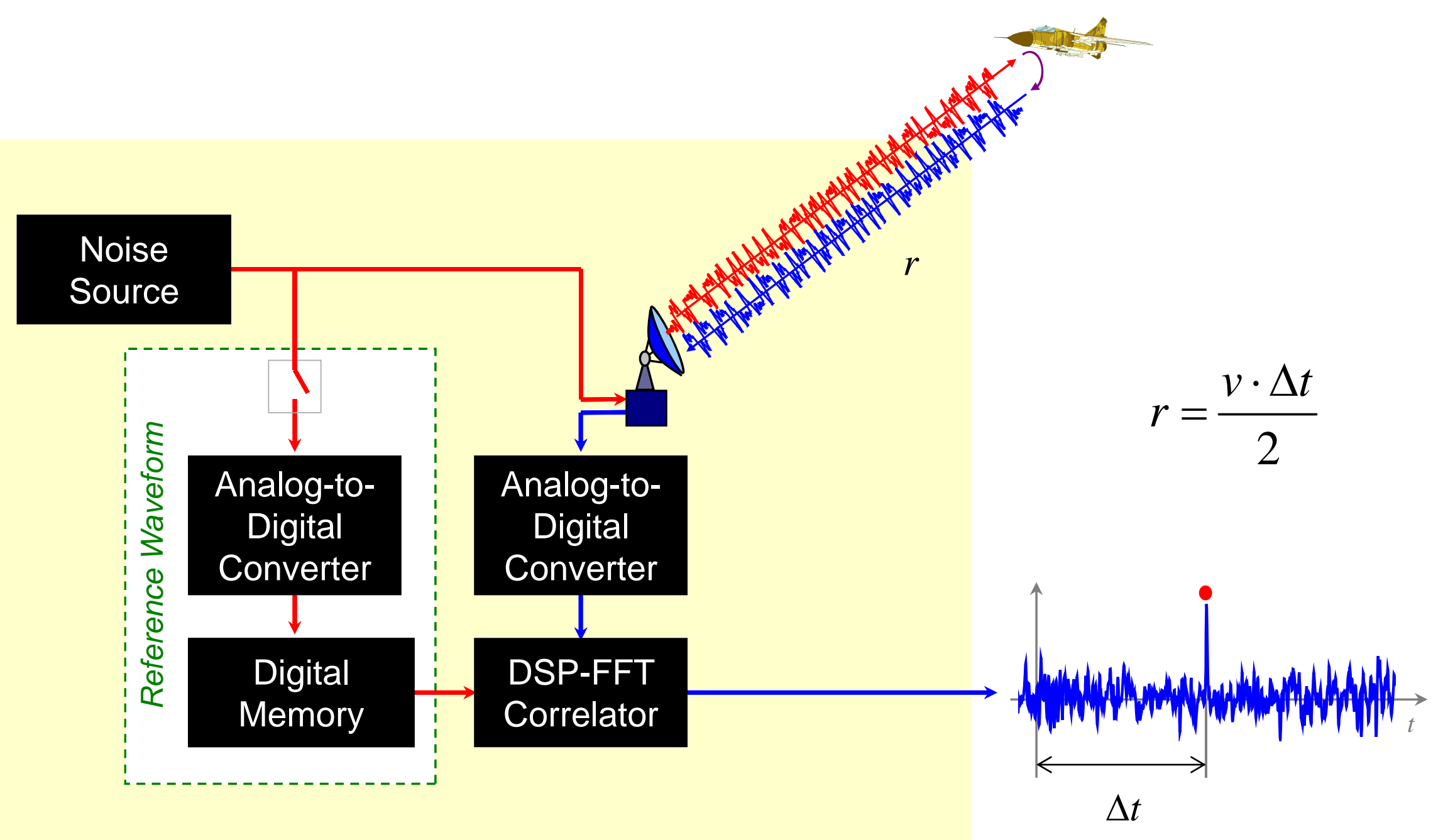




\section{Chaos Noise Radar}

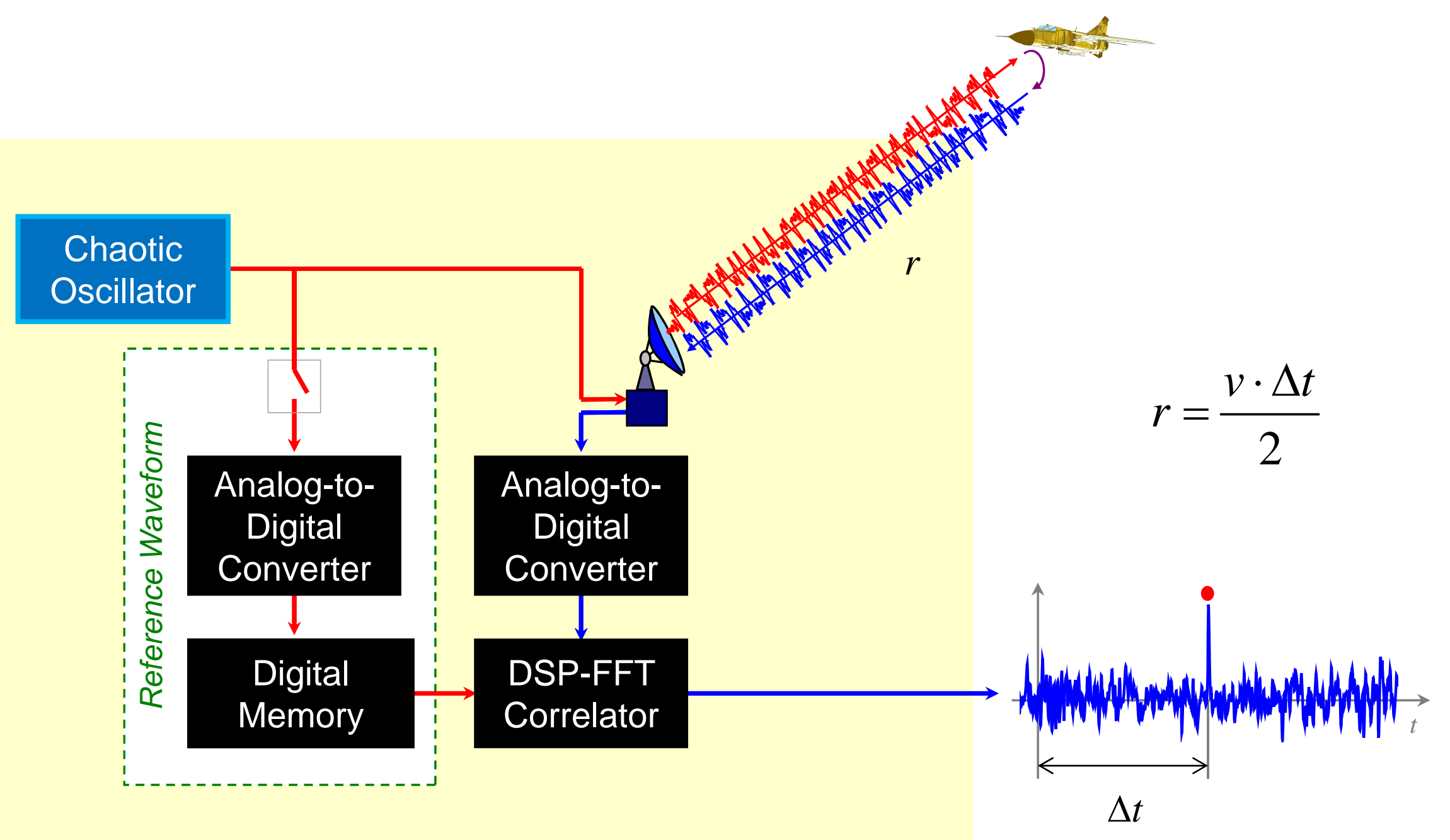




\section{Chaos Noise Radar}

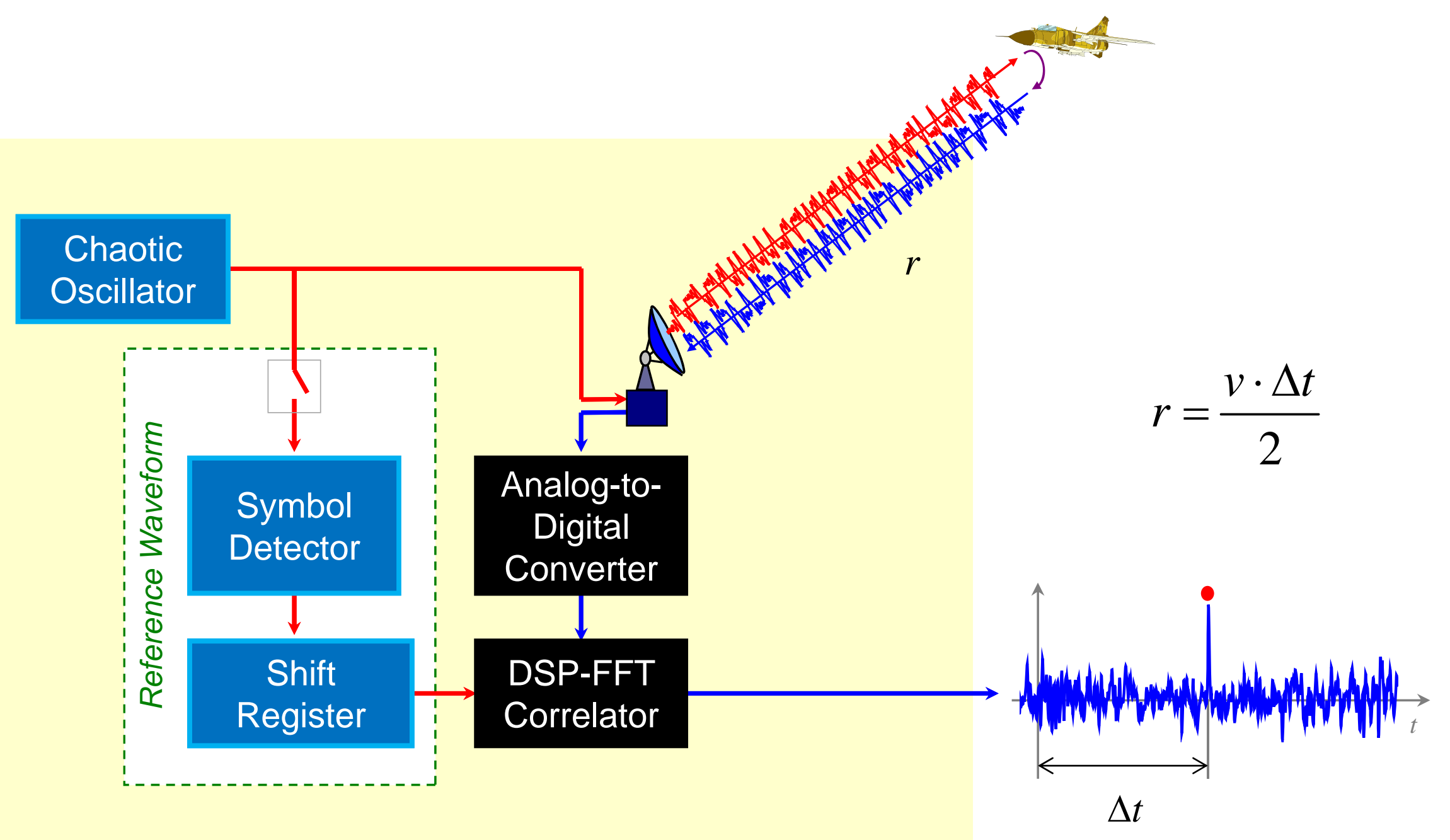




\section{Chaos Radar}

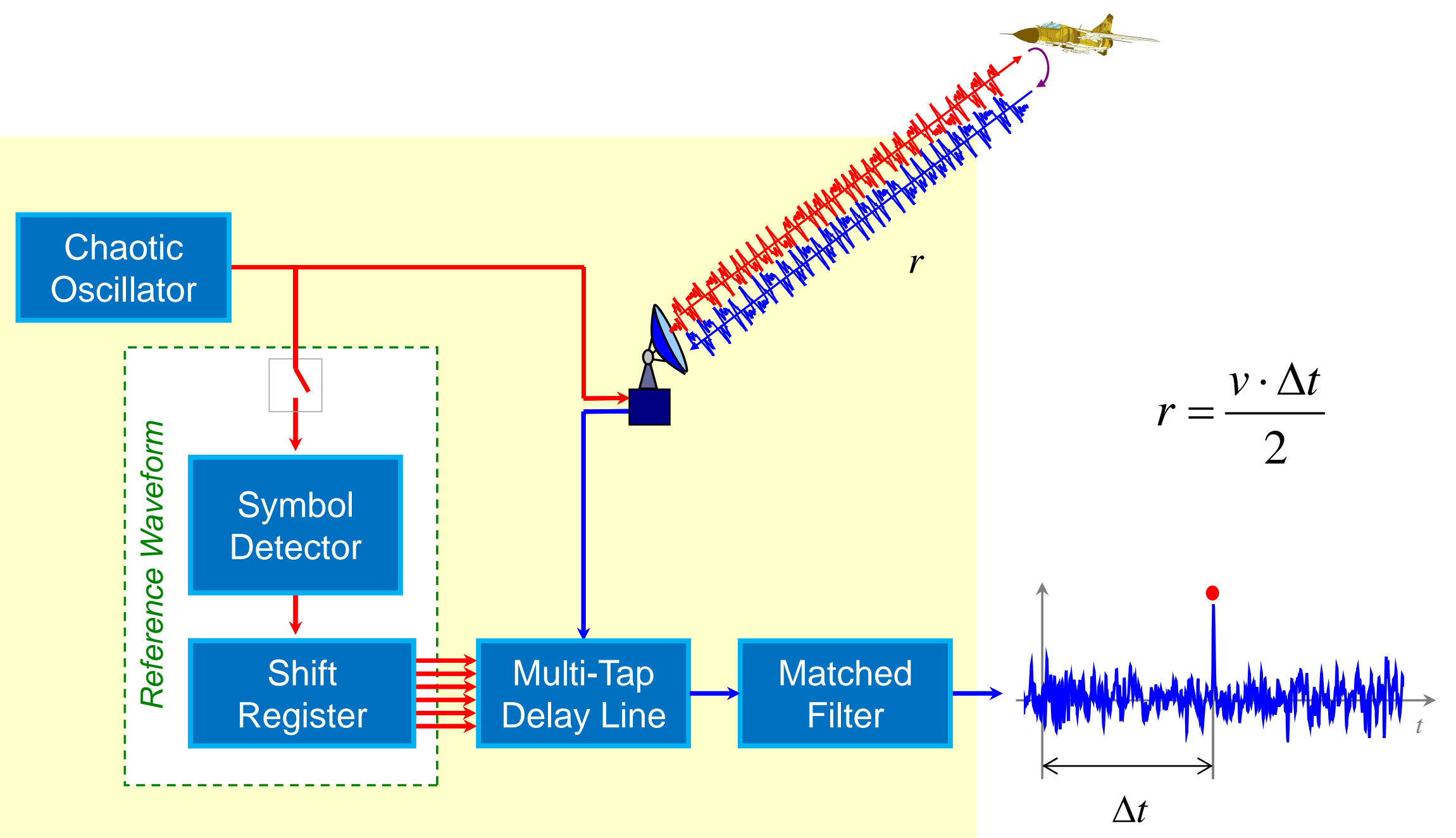




\section{Conclusions}

- Acoustic Ranging Using Solvable Chaos

- Hybrid Oscillator

- binary symbols

- basis pulse

- Matched Filter

- Exploits Chaos in Meaningful Way

- Simple, Low-Cost Components

- Concept for Low-Cost UWB Radar

- No Analog-to-Digital Conversion

- No DSP-FFT Correlations

- Reduced Sampling and Storage

- Matched Filter Performance
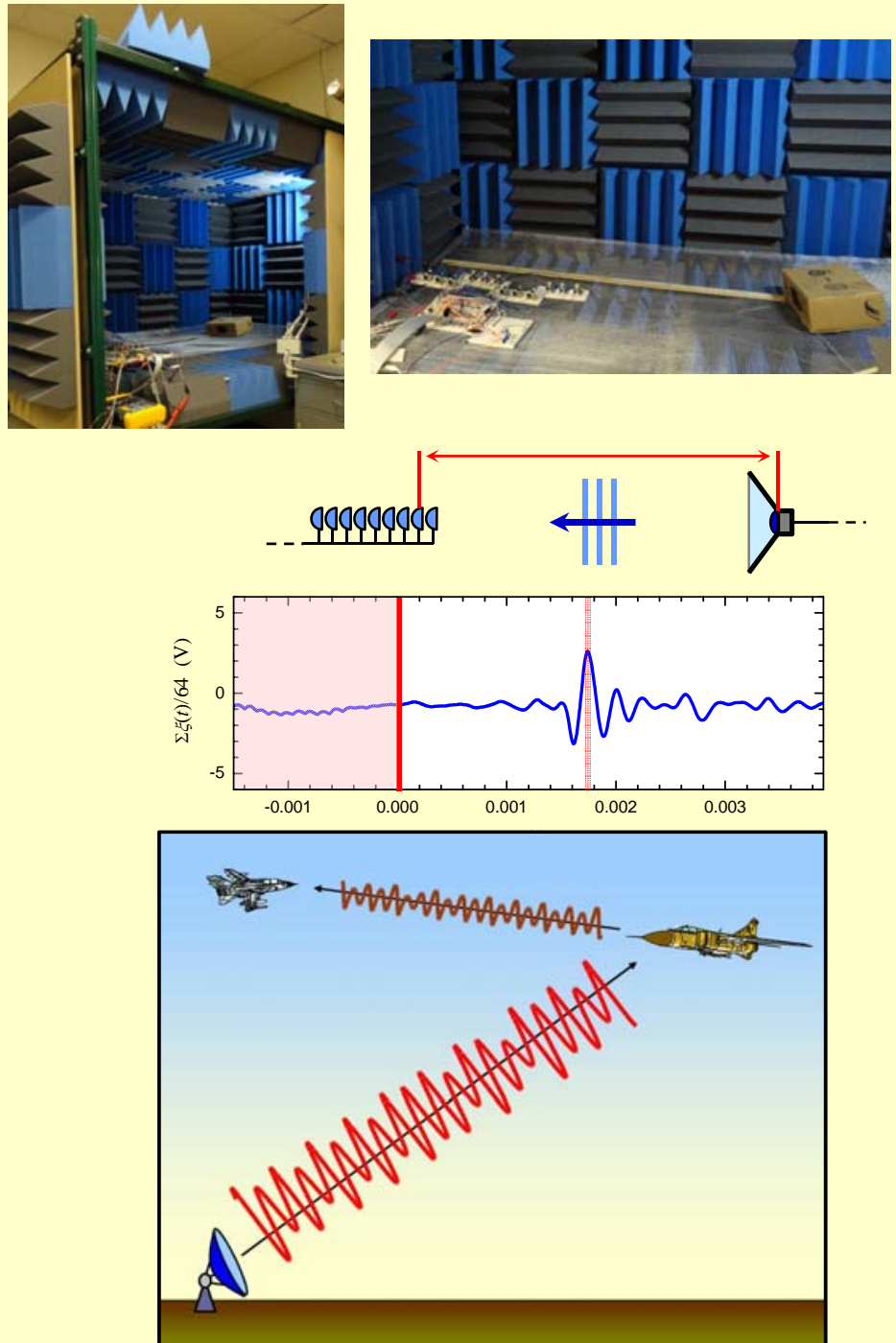


\section{Extra Slides}




\section{Transformed Basis}

- Square pulse

$$
\phi(t)=\left\{\begin{array}{lc}
1, & 0 \leq t<1 \\
0, & \text { otherwise }
\end{array}\right.
$$

- Linear transformation

$$
P(t)=\int_{-\infty}^{\infty} \phi(\tau) \cdot \rho(t-\tau) d \tau
$$

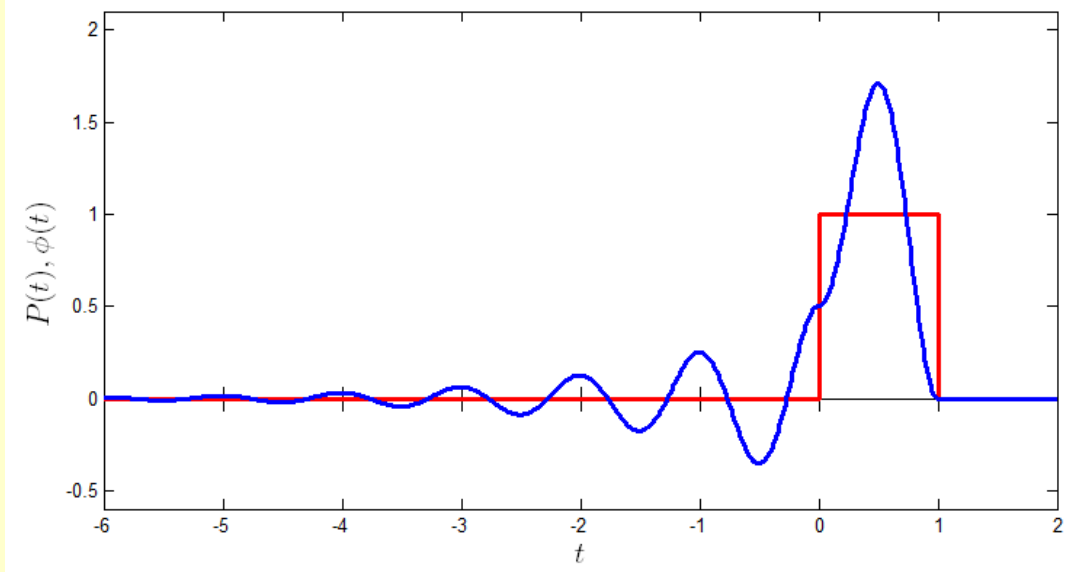

- Matched filter

$$
P(t)=\left\{\begin{array}{cc}
\frac{1}{2} \cdot\left[\cos (\omega t)-\frac{\beta}{\omega} \sin (\omega t)\right] \cdot 2^{t}, & t \leq 0 \\
1-\frac{1}{2} \cdot\left[\cos (\omega t)-\frac{\beta}{\omega} \sin (\omega t)\right] \cdot 2^{t}, & 0<t \leq 1 \\
0, & 1<t
\end{array}\right.
$$$$
M_{2} \circ \rho(-t)=P(-t)
$$$$
M_{2} \circ\left(M_{1} \circ \delta(t)\right)=P(-t)
$$ 


\section{Differential Equation}

- Waveform...

$$
v(t)=\sum_{m=-\infty}^{\infty} s_{m} \cdot P(t-m)
$$

... satisfies the differential equation

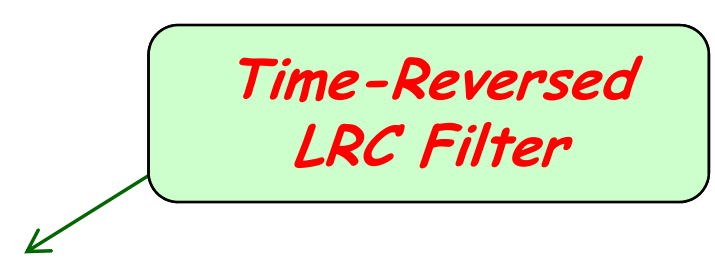

$$
\frac{d^{2} v}{d t^{2}}-2 \beta \frac{d v}{d t}+\left(\omega^{2}+\beta^{2}\right)\left(v-v_{s}\right)=0
$$

... where

$$
v_{S}(t)=\sum_{m=-\infty}^{\infty} s_{m} \cdot \phi(t-m)
$$

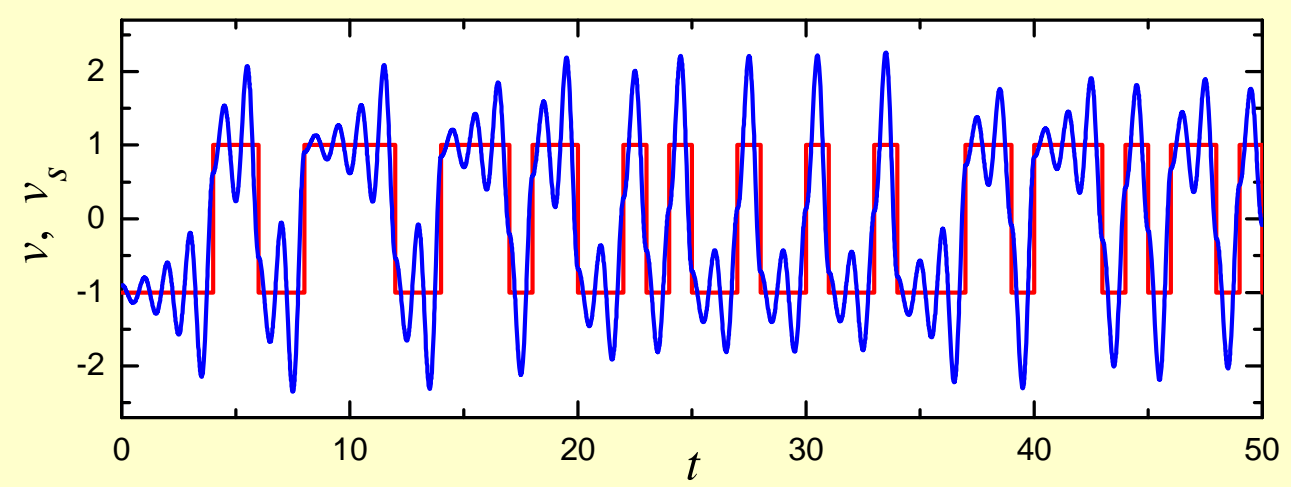




\section{Guard Condition}

- Waveform also satisfies...

$$
\frac{d v}{d t}=0 \Rightarrow v_{s}=\operatorname{sgn}(v)
$$

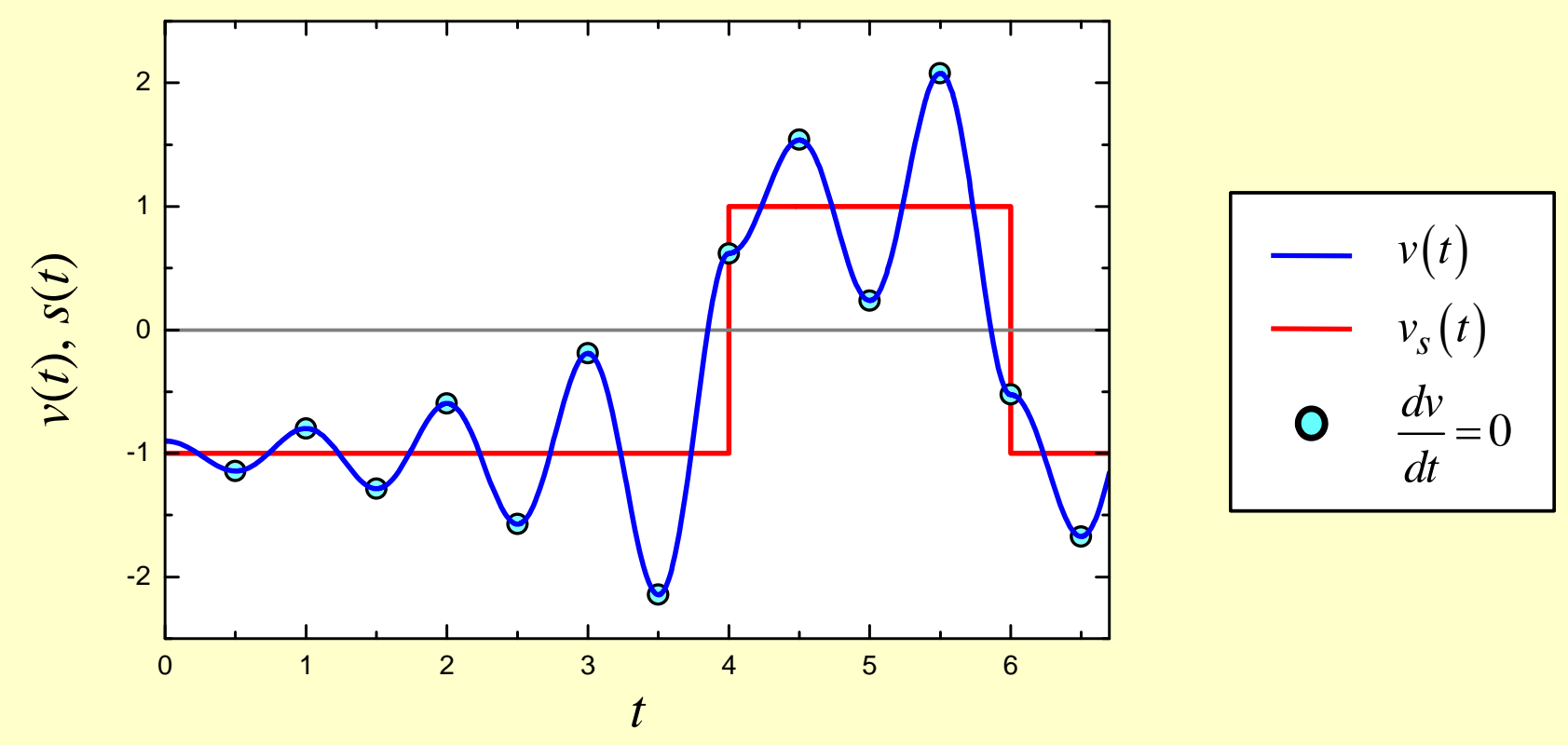




\section{Chaos}

- Interpret as a dynamical system...

$$
\begin{gathered}
\frac{d^{2} v}{d t^{2}}-2 \beta \frac{d v}{d t}+\left(\omega^{2}+\beta^{2}\right) \cdot\left(v-v_{s}\right)=0 \\
\frac{d v}{d t}=0 \Rightarrow v_{s}=\operatorname{sgn}(v)
\end{gathered}
$$

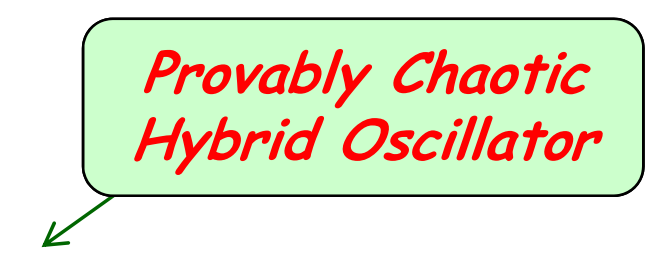

T. Saito and H. Fujita, "Chaos in a manifold linear system," Electron. Commun. Jpn. 64A, 9 (1981).

\section{Chaotic Electronic Oscillator}
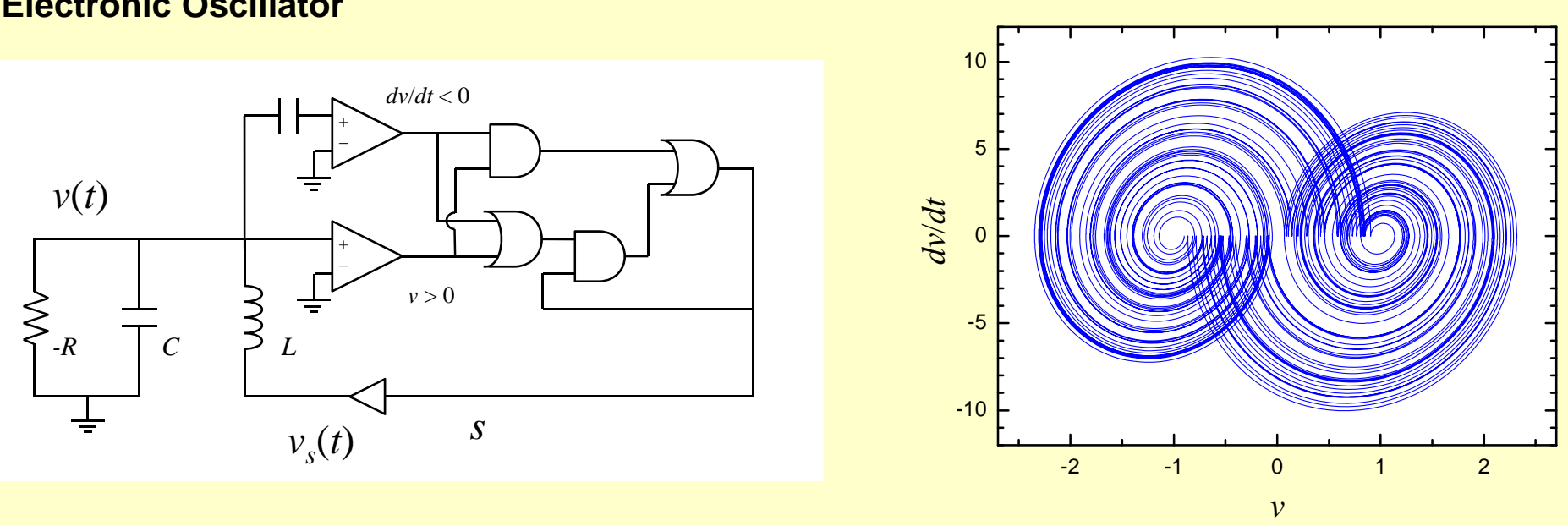


\section{Oscillator Model}

$$
\begin{gathered}
\frac{d^{2} v}{d t^{2}}-2 \beta \frac{d v}{d t}+\left(\omega^{2}+\beta^{2}\right) \cdot\left(v-v_{s}\right)=0 \\
\frac{d v}{d t}=0 \Rightarrow v_{s}=\operatorname{sgn}(v)
\end{gathered}
$$

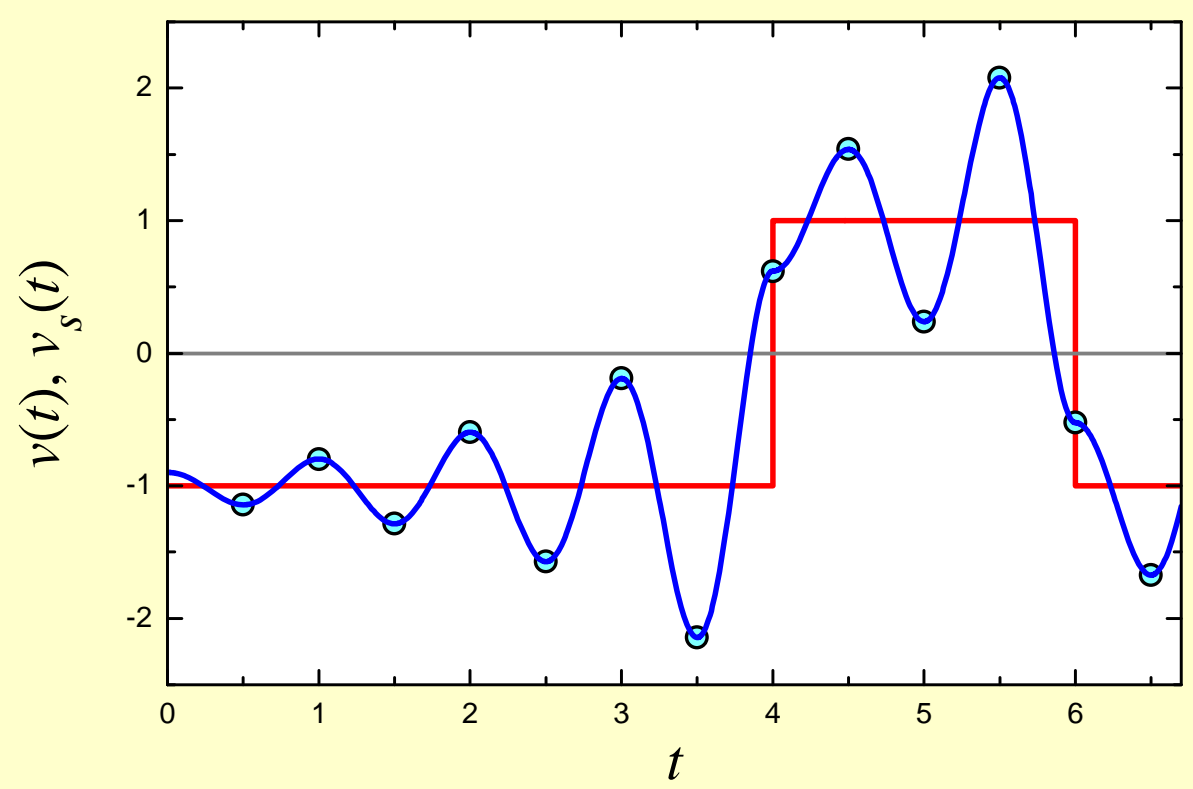

$$
\begin{array}{ll}
- & v(t) \\
- & v_{S}(t) \\
\text { ○ } & \frac{d v}{d t}=0
\end{array}
$$

$$
\begin{gathered}
0<\beta \leq \ln 2 \\
\omega=2 \pi
\end{gathered}
$$

T. Saito and H. Fujita, "Chaos in a manifold linear system," Electron. Commun. Jpn. 64A, 9 (1981). 


\section{Analytic Solution}

- Model admits an exact solution (easily verified):

$$
v(t)=\sum_{m=-\infty}^{\infty} s_{m} \cdot P(t-m), \quad v_{s}(t)=\sum_{m=-\infty}^{\infty} s_{m} \cdot R(t-m)
$$

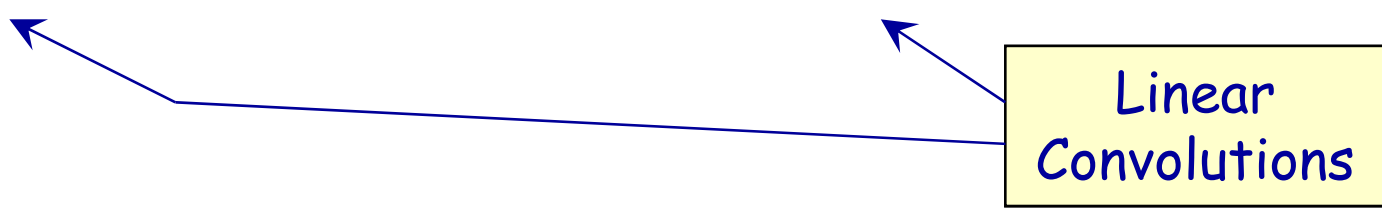

where each $s_{m}= \pm 1$ and...

$$
\begin{aligned}
& P(t)=\left\{\begin{array}{ll}
\left(1-e^{-\beta}\right) e^{\beta t}\left(\cos \omega t-\frac{\beta}{\omega} \sin \omega t\right), & t<0 \\
1-e^{\beta(t-1)\left(\cos \omega t-\frac{\beta}{\omega} \sin \omega t\right),} & 0 \leq t<1
\end{array} \quad R(t)=\left\{\begin{array}{lc}
0, & t<0 \\
1, & 0 \leq t<1 \\
0, & 1 \leq t
\end{array}\right.\right. \\
& 0 \text {, } \\
& 1 \leq t
\end{aligned}
$$

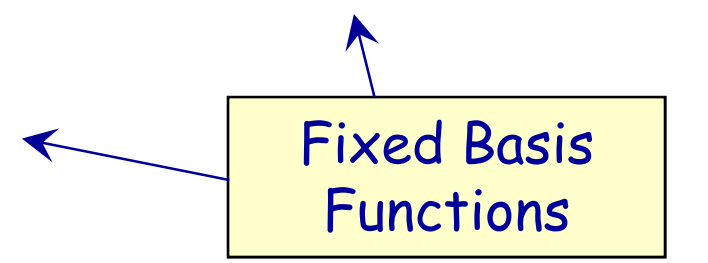




\section{Symbols vs. Nyquist Sampling}

- Nyquist-Shannon sampling requirement

at least 2 samples per cycle,
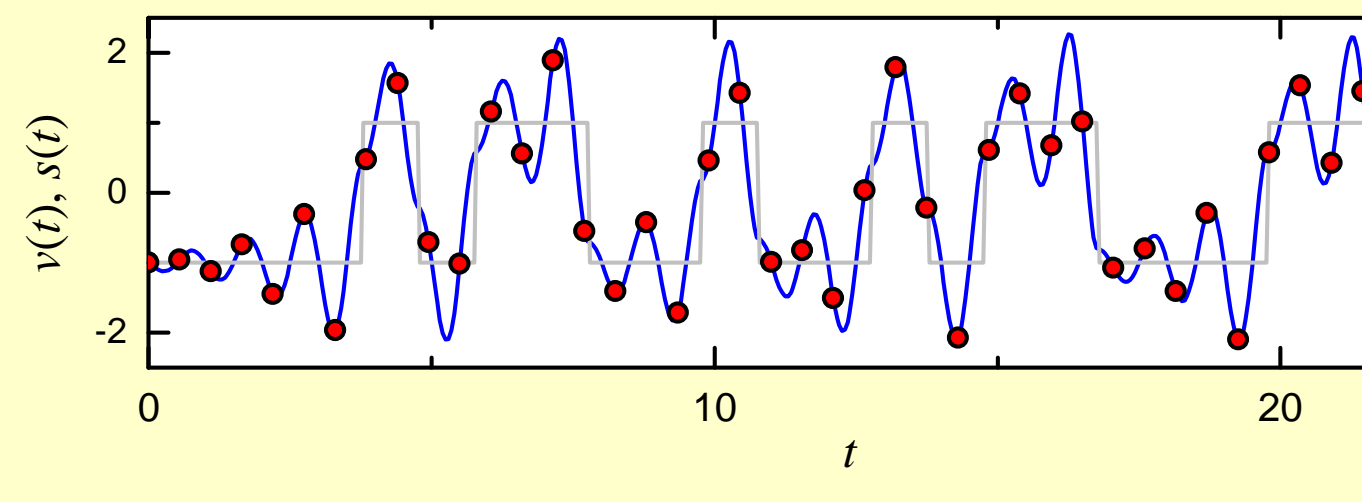

$N_{b}$ bits per sample

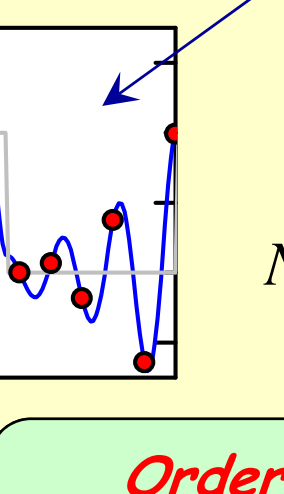

$f_{s}>2 B$
$N_{b}=8,12,16, \ldots$ ???

Reduction in Sampling and

Storage Requirements

- Symbolic representation

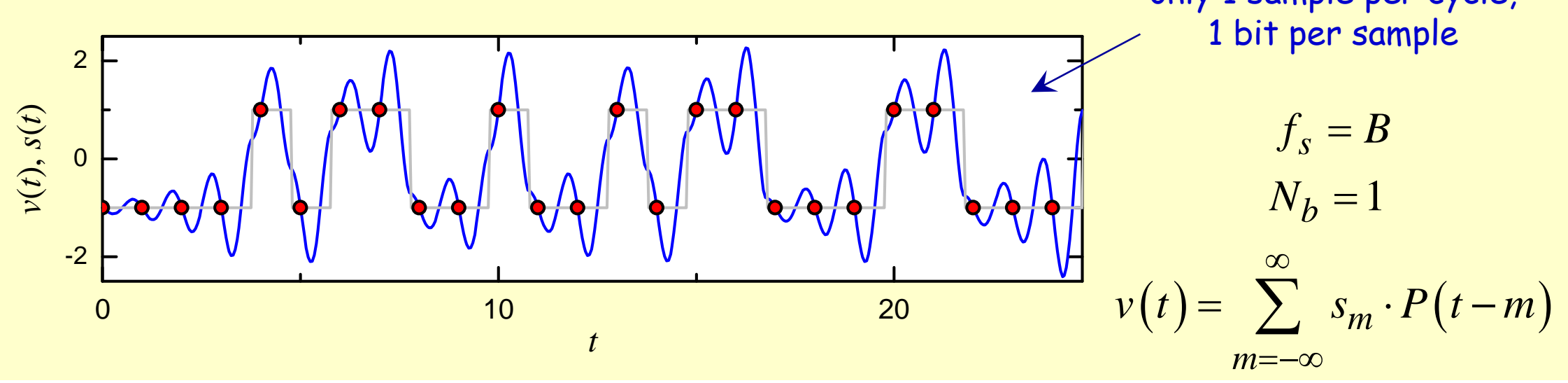




\section{Correlation Receiver}

- Channel Model

$$
v(t-\Delta t) \longrightarrow \underset{\substack{\uparrow \\ w(t) \text { (noise) }}}{\longrightarrow} \stackrel{v(t)}{\rightarrow} \xi(t)
$$

- Correlation Receiver

- optimal linear filter for detecting a given signal in additive white Gaussian noise (AWGN)

$$
\begin{aligned}
& \xi(t)=\int_{-\infty}^{\infty} \tilde{v}(\tau) \cdot v_{r e f}(\tau-t) d \tau \\
& v_{r e f}(t)=\sum_{m=1-N}^{0} s_{m} \cdot P(t-m)
\end{aligned}
$$

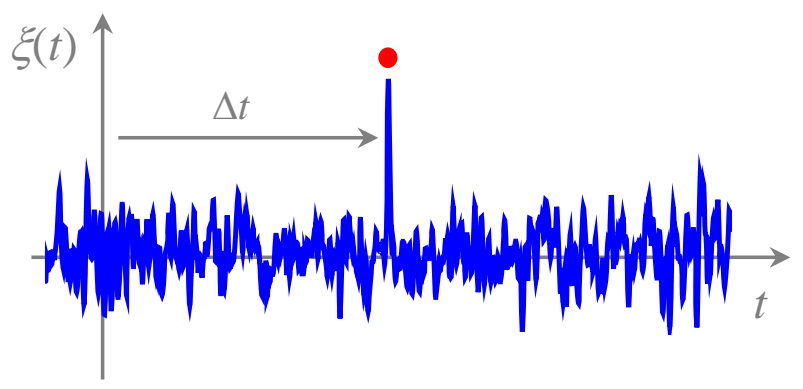




$$
\begin{aligned}
& \xi(t)=\int_{-\infty}^{\infty} \tilde{v}(\tau) \cdot v_{\text {ref }}(\tau-t) d \tau \\
& =\int_{-\infty}^{\infty} \tilde{v}(\tau) \cdot\left\{\sum_{m=1-N}^{0} s_{m} \cdot P(\tau-t-m)\right\} d \tau \\
& =\sum_{m=1-N}^{0} s_{m} \cdot \int_{-\infty}^{\infty} \tilde{v}(\tau) \cdot P(\tau-t-m) d \tau \\
& =\sum_{m=1-N}^{0} s_{m} \cdot \int_{-\infty}^{\infty} \tilde{v}(\tau+m) \cdot P(\tau-t) d \tau \\
& \xi(t)=\int_{-\infty}^{\infty}\left\{\sum_{m=1-N}^{0} s_{m} \cdot \tilde{v}(\tau+m)\right\} \cdot P(\tau-t) d \tau
\end{aligned}
$$

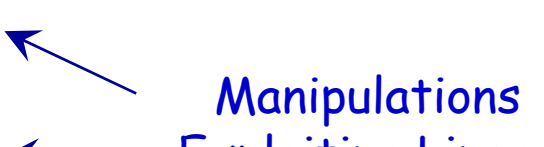




\section{Matched Filter for Basis Function}

- Linear Filter

$$
\mathrm{L} \circ \tilde{v}(t)=\int \tilde{v}(\tau) P(\tau-t) d \tau
$$

- impulse response is time reversal of basis function...

- Basis Function

$$
P(t)
$$

- Matched Filter

$$
\mathrm{L} \circ \delta(t)=P(-t)
$$
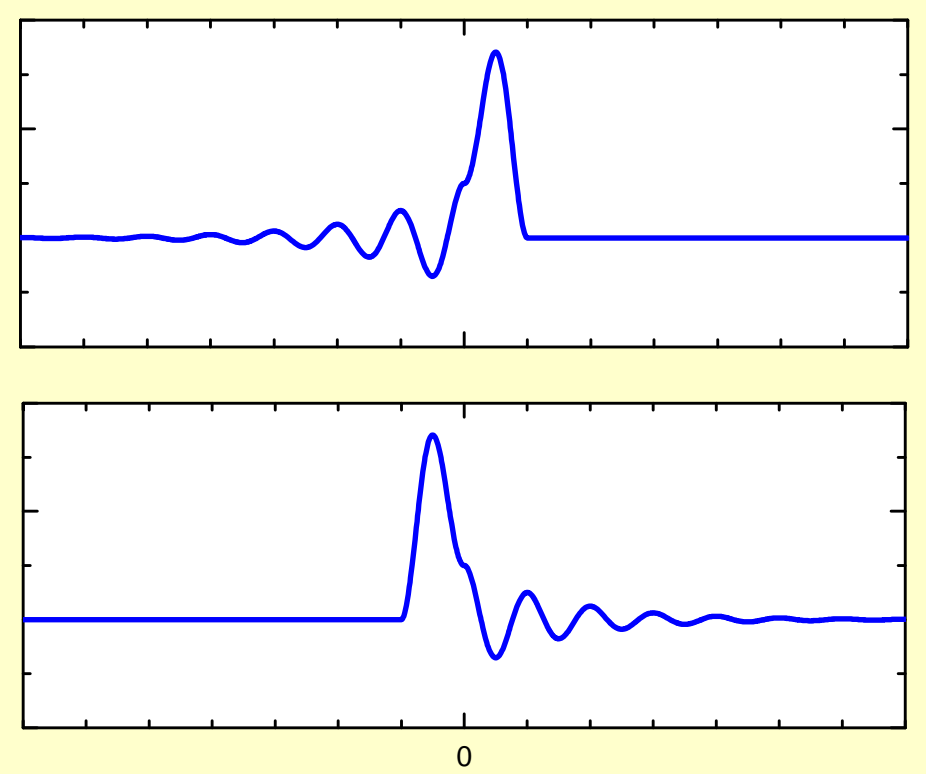

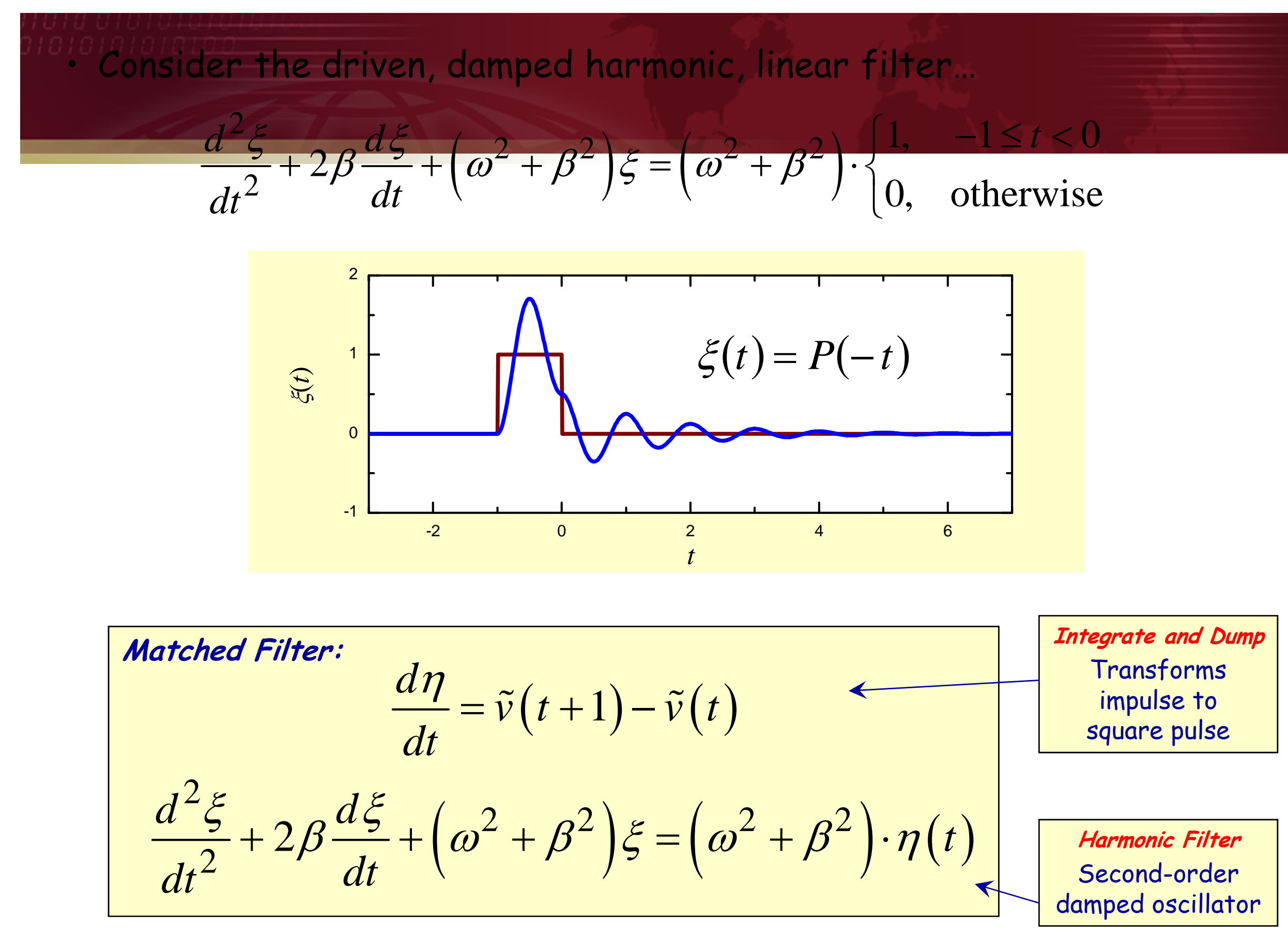


$$
\begin{aligned}
& \text { Matched Filter: } \\
& \qquad \frac{d \eta}{d t}=\tilde{v}(t+1)-\tilde{v}(t) \\
& \frac{d^{2} \xi}{d t^{2}}+2 \beta \frac{d \xi}{d t}+\left(\omega^{2}+\beta^{2}\right) \xi=\left(\omega^{2}+\beta^{2}\right) \cdot \eta(t)
\end{aligned}
$$

- Impulse Response

$$
\tilde{v}(t)=\delta(t) \Rightarrow \begin{aligned}
& \eta(t)=\phi(-t) \\
& \xi(t)=P(-t)
\end{aligned}
$$

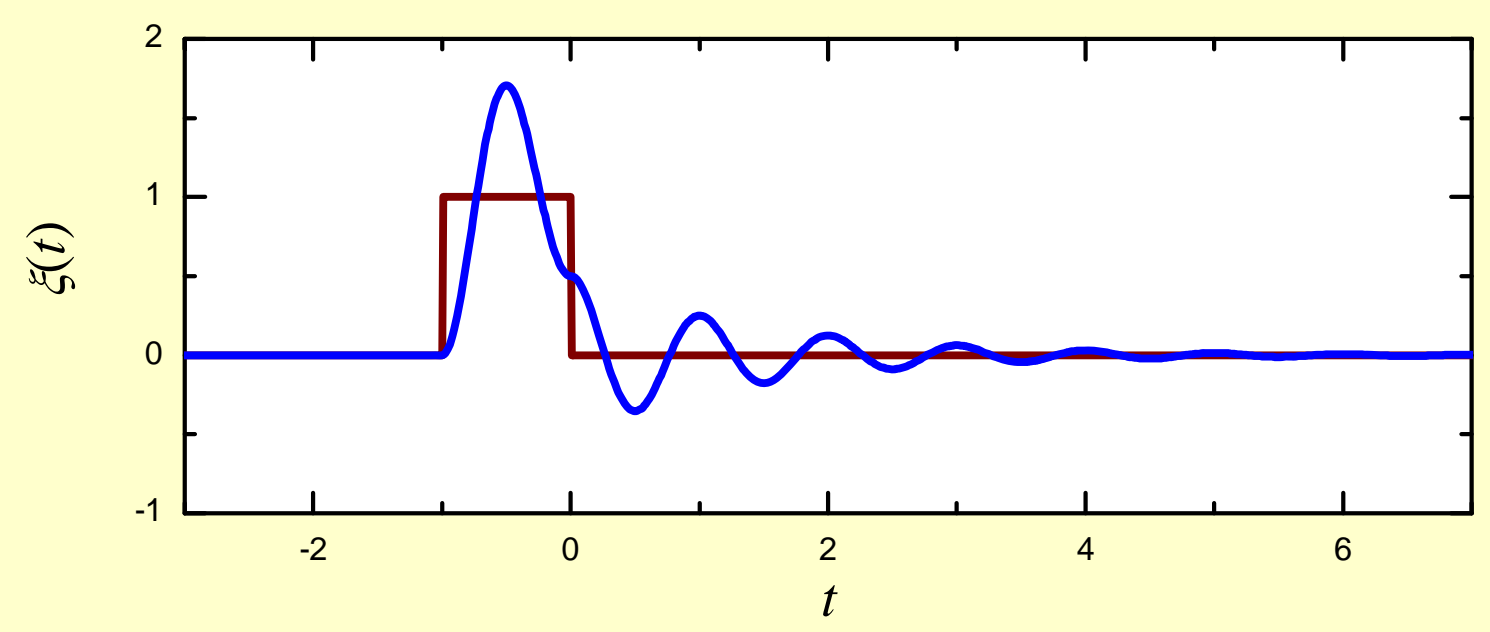




\section{Correlation Receiver}

$$
v_{\text {ref }}(t)=P(t)
$$

$$
v_{\text {ref }}(t)=\sum_{m=1-N}^{0} s_{m} \cdot P(t-m)
$$

\section{Matched Filter}

Integrate and Dump:

$$
\frac{d \eta}{d t}=\tilde{v}(t+1)-\tilde{v}(t)
$$

Harmonic Filter:

$$
\begin{array}{r}
\frac{d^{2} \xi}{d t^{2}}+2 \beta \frac{d \xi}{d t}+\left(\omega^{2}+\beta^{2}\right) \xi \\
=\left(\omega^{2}+\beta^{2}\right) \cdot \eta(t)
\end{array}
$$

\section{Correlation Receiver}

Integrate and Dump:

$\frac{d \eta}{d t}=\sum_{m=1-N}^{0} s_{m} \cdot\{\tilde{v}(t+1+m)-\tilde{v}(t+m)\}$

Harmonic Filter:

$$
\begin{array}{r}
\frac{d^{2} \xi}{d t^{2}}+2 \beta \frac{d \xi}{d t}+\left(\omega^{2}+\beta^{2}\right) \xi \\
=\left(\omega^{2}+\beta^{2}\right) \cdot \eta(t)
\end{array}
$$




\section{Correlation Receiver}

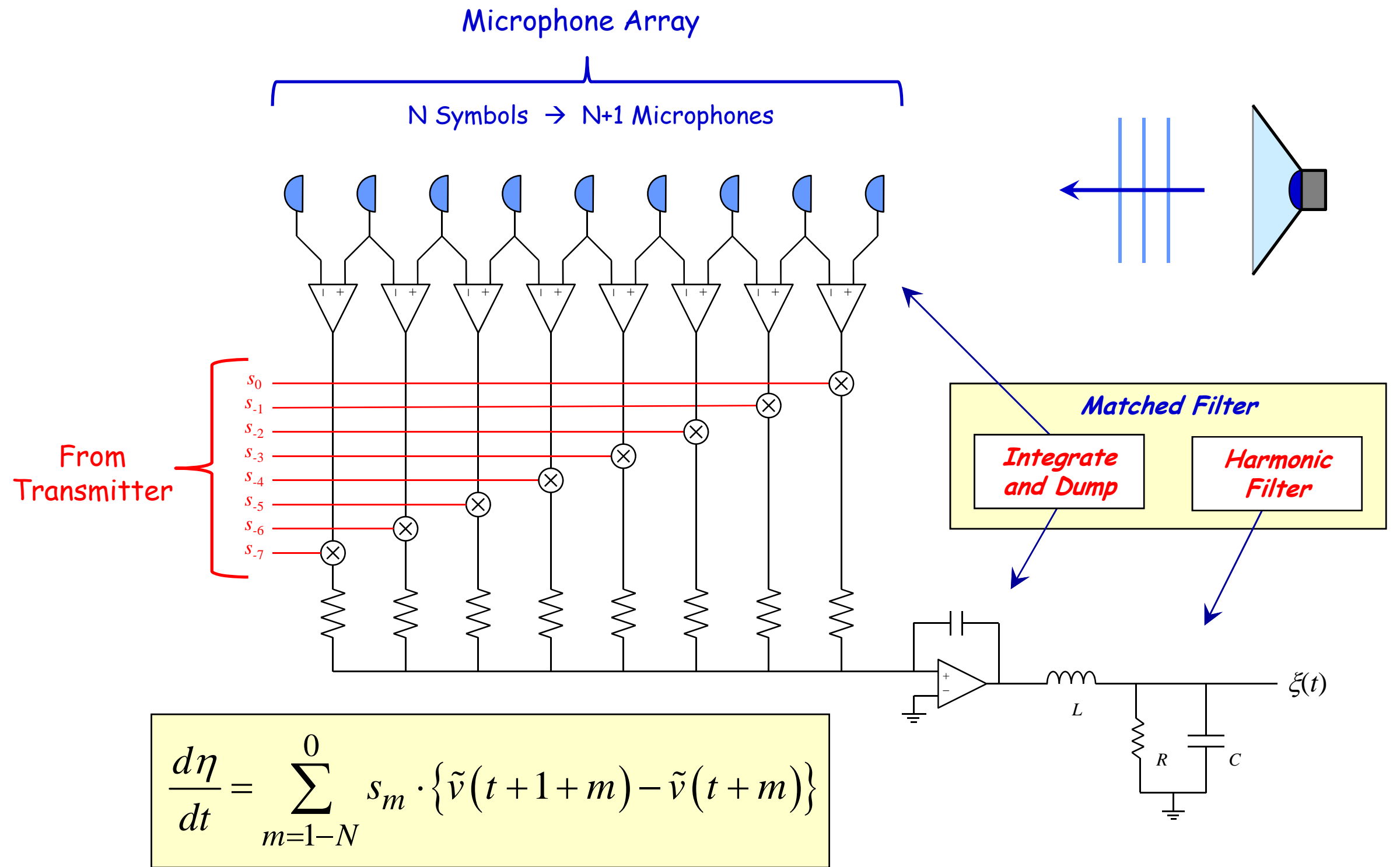

